\title{
Global gravitational anomalies and transport
}

\author{
Subham Dutta Chowdhury and Justin R. David \\ Centre for High Energy Physics, Indian Institute of Science, \\ C. V. Raman Avenue, Bangalore 560012, India \\ E-mail: subham@cts.iisc.ernet.in, justin@cts.iisc.ernet.in
}

\begin{abstract}
We investigate the constraints imposed by global gravitational anomalies on parity odd induced transport coefficients in even dimensions for theories with chiral fermions, gravitinos and self dual tensors. The $\eta$-invariant for the large diffeomorphism corresponding to the $T$ transformation on a torus constraints the coefficients in the thermal effective action up to mod 2. We show that the result obtained for the parity odd transport for gravitinos using global anomaly matching is consistent with the direct perturbative calculation. In $d=6$ we see that the second Pontryagin class in the anomaly polynomial does not contribute to the $\eta$-invariant which provides a topological explanation of this observation in the 'replacement rule'. We then perform a direct perturbative calculation for the contribution of the self dual tensor in $d=6$ to the parity odd transport coefficient using the Feynman rules proposed by Gaumé and Witten. The result for the transport coefficient agrees with that obtained using matching of global anomalies.
\end{abstract}

Keywords: Anomalies in Field and String Theories, Chern-Simons Theories, Field Theories in Higher Dimensions, Effective field theories

ARXIV EPRINT: 1604.05003 


\section{Contents}

1 Introduction 1

2 Global anomalies and thermal effective action 4

$3 \quad$ Global anomalies and transport in $d=2 \quad 6$

3.1 Fermions 9

$\begin{array}{lll}3.2 & \text { Chiral bosons } & 11\end{array}$

$\begin{array}{lll}3.3 \text { Gravitinos } & 12\end{array}$

4 Global anomalies and transport in $d=6 \quad 13$

$\begin{array}{lll}4.1 \text { Fermions } & 17\end{array}$

$\begin{array}{lll}4.2 & \text { Gravitinos } & 18\end{array}$

$\begin{array}{lll}4.3 & \text { Self-dual tensors } & 19\end{array}$

5 Transport for self dual tensors in $d=6$ at one loop 20

6 Conclusions 23

A Curvature integrals and Chern-Simons terms 24

$\begin{array}{ll}\text { B Correlators of self dual tensors in } d=6 & 27\end{array}$

$\begin{array}{lll}\text { B.1 Evaluating the non-vanishing contributions } & 38\end{array}$

C Summary of $\eta$ invariants in various dimensions 48

\section{Introduction}

Several recent works have investigated the relationship between parity odd transport coefficients and anomalies in even dimensions [1-11]. Among these relations, the ones relating the mixed gravitational anomalies or the pure gravitational anomalies to the appropriate parity odd transport coefficients are the harder to establish. This is because these anomalies influence transport coefficients which occur at lower order in the derivative expansion when compared to the order they occur in the anomalous conservation law. There are three methods used to establish the relationship between the gravitational anomalies and the corresponding transport coefficients

1. Direct perturbative evaluation of the Kubo formula of the transport coefficients using finite temperature field theory methods [12-16].

2. Using the method of consistency of the Euclidean vacuum $[17,18]$. 
3. Evaluating the one loop thermal partition functions of the theory on a spatial slice to obtain an effective Chern-Simons terms which are in turn related to the transport coefficients $[15,19]$.

Recently a new method has been proposed by [20] which relies on matching anomalies of large diffeomorphisms or large gauge transformations to fix the Chern-Simons terms in the thermal effective action. One of the goals of this paper is study this approach in more detail, extend the method to $d=6$ and study the situations in which there are gravitinos and self dual tensors in the theory. A second aim of the paper is to evaluate the contribution of self dual tensors to parity odd transport coefficient in $d=6$ perturbatively using the Feynman rules for these fields put forward by [21]. This calculation will also check the consistency of the result obtained using the matching global anomalies.

Let us briefly summarize the method of global anomaly matching to determine the thermal effective action. Consider a $2 n$ dimensional manifold torus $T^{2 n}$. We will identify one of the directions of the torus to play the role of Euclidean time. Let the metric on the torus be $g_{\mu \nu}$ on which there exists a global diffeomorphism

$$
g_{\mu \nu} \rightarrow g_{\mu \nu}^{T}
$$

If there exists a global anomaly, the partition function of the theory changes by

$$
Z\left[g_{\mu \nu}\right] \rightarrow Z\left[g_{\mu \nu}^{T}\right]=e^{-i \pi \eta} Z\left[g_{\mu \nu}\right]
$$

where $\eta$ is the $\eta$-invariant ${ }^{1}$ corresponding to the global diffeomorphism. We will consider the $T$-symmetry of the torus. After evaluating the $\eta$-invariant, one then writes down a thermal effective action involving the components of the metric which transforms identical to (1.2). The thermal effective action is in general a Chern-Simons type action considered in all the remaining $2 n-1$ directions excluding time. It is clear from (1.2) that coefficients in any action determined this way will be ambiguous up to mod 2. Once the effective action is obtained, we can take the decompactification limit in the $2 n-1$ directions and use it to obtain response functions corresponding to the transport coefficients.

The contributions of chiral gravitinos to parity odd transport coefficients in the theory are particularly tricky to determine. As noted in [17] the method of consistency of the Euclidean vacuum cannot be applied to determine the contribution of chiral gravitinos to parity odd transport. It was argued in [17] that this can be attributed to the difference in the number of zero modes for the gravitinos on the plane versus the cone. This was confirmed by evaluating the expectation of the thermal helicity [24] for theories with gravitinos and later in [16], by the direct perturbative evaluation of the contribution of gravitinos to the transport coefficient using the Kubo formula. To highlight this fact, let us naively apply the method of consistency of the Euclidean vacuum $[17,18]$ to determine the contribution of gravitinos to transport coefficients. A naive application of the method, predicts that the transport coefficients of gravitinos are directly related to the gravitational anomaly by a multiplicative constant. For example consider the case of $d=2$, and let $\lambda^{(2)}=\tilde{c}_{2 d} T^{2}$ be

\footnotetext{
${ }^{1}$ The details of how the topological $\eta$-invariant is defined and evaluated will be discussed subsequently.
} 
the parity odd coefficient due to the presence of chiral gravitinos, then a naive application of the method of consistency of the Euclidean vacuum predicts the relation

$$
\tilde{c}_{2 d}=-8 \pi^{2} c_{g}
$$

where $c_{g}$ is the gravitational anomaly due to chiral gravitinos. However, direct perturbative calculations [16] and the expectation value of thermal helicity [24] reveal that the the contribution of chiral gravitinos in $d$ dimensions to transport is equal to that of $d-1$ chiral fermions. In this paper we use the method of matching global anomalies to determine the contribution of chiral gravitinos to transport. We see that result from matching global anomalies is consistent with the direct perturbative calculation up to mod 2. This phenomenon is consistently seen for gravitino like theories in $d=2$ and gravitinos in $d=6$.

Let us now examine another aspect of the replacement rule put forward in $[17,18]$ and the observations in the works of [22-24]. For theories in which the replacement rule can be applied, namely theories without gravitinos but can contain chiral fermions and self dual tensors, it is seen that second or higher Pontryagin classes in the anomaly polynomial of a chiral field does not contribute to the transport coefficient. This was summarized succinctly by the 'replacement' rule. Lets recall the rule for the transport coefficient which is sensitive to pure gravitation anomalies in $d=6$. Now we restrict our arguments to theories which contain only chiral fermions and self dual tensors. Consider the anomaly polynomial for pure gravitational anomalies in $d=6$,

$$
\begin{aligned}
\mathcal{P}_{d=6} & =c_{\gamma}\left(\operatorname{Tr}(\hat{R})^{2}\right)^{2}+c_{\delta}\left(\frac{1}{4} \operatorname{Tr}\left(\hat{R}^{4}\right)-\frac{1}{8} \operatorname{Tr}\left(\hat{R}^{2}\right)^{2}\right), \\
\hat{R}_{a b} & =\frac{1}{2} R_{a b c d} d x^{c} \wedge d x^{d} .
\end{aligned}
$$

Note $c_{\gamma}$ is the coefficient which occurs with the square of the first Pontryagin class while the $c_{\delta}$ occurs with the second Pontryagin class. Now let the parity odd transport coefficient determined by the three point function of the stress tensor be parametrised as

$$
\lambda_{3}^{(6)}=9 \tilde{c}_{g}^{6 d} T^{4} .
$$

Then 'replacement rule' predicts the relation

$$
\tilde{c}_{g}^{6 d}=-\left(8 \pi^{2}\right)^{2} c_{\gamma}
$$

To summarize the replacement rule predicts that second Pontryagin class does not contribute. Using the method of global anomalies to determine the thermal effective action and $\lambda_{3}^{(6)}$, we see the $\eta$-invariant corresponding to the $T$-symmetry of the torus for Weyl fermions, and self dual-tensors do not receive any topological contribution form the second Pontryagin class for theories in $d=6$ and therefore they do not contribute to transport. Thus the method of global anomaly matching provides a topological explanation for this observation in the replacement rule. We show that the prediction (1.6) is consistent with global anomaly matching for Weyl fermions, self-dual tensors. We will also explicitly verify the prediction in (1.6) by performing a direct perturbative evaluation of the relevant Kubo 
formula using Feynman rules for the self dual tensor given in [21]. We show that indeed the contribution of the self dual tensor indeed agrees with that predicted by replacement rule.

Though our paper focuses on contributions to transport coefficients occuring from pure gravitational anomalies, the analysis can be generalized to case of mixed anomalies. In fact [20] studies the case of mixed anomalies in $d=4$.

The organization of the paper is as follows. In section 2 we briefly review the method put forward by [20] to set our notations. In section 3 we study the $d=2$ case in detail for all the chiral fields. Since the partition function and modular properties under the $T$ symmetry of free chiral fields in $d=2$ are known exactly we also compare the calculation of the $\eta$-invariant to these results. We pay particular attention to the spin structure which is picked up by the $\eta$-invariant. We extend the analysis to $d=6$ in section 4 . We then proceed to evaluate the transport coefficient $\lambda_{3}^{(6)}$ for self dual tensors using the propagator of [21] in section 5. Section 6 contains our conclusions. Appendix A contains the details involved in evaluating the $\eta$-invariants and appendix B contains the details of Wick contractions manipulations and simplification of the correlators in the evaluation of the the Kubo formula for $\lambda^{(6)}$ for the self dual tensor in $d=6$. Finally appendix $\mathrm{C}$ summarises the $\eta$ invariants corresponding to the $T^{2}$ transformation for chiral matter in $d=2,6,10$.

\section{Global anomalies and thermal effective action}

In this section we will review the method introduced by [20] to constrain thermal effective actions using global anomalies. This section will provide the outline of the logic of the method using chiral fermions in $d=2$ as an example. This method will be implemented in detail in section 3 . We will also generalize this method to theories which contain self dual and gravitinos tensors in $d=2, d=6$

Consider a theory of complex Weyl fermions in $d=2$ on a torus $\hat{T}^{2}$. Let the coordinates on the torus be given by $(t, x)$, with the identifications

$$
(t, x) \sim(t+2 \pi n, x+2 \pi m) .
$$

Let the metric on the torus be given by

$$
g: \quad d s^{2}=(d t+a(x) d x)^{2}+d x^{2} .
$$

Lets now consider the large diffeomorphism of the torus generated by the transformation

$$
\left(\begin{array}{l}
t \\
x
\end{array}\right) \rightarrow\left(\begin{array}{ll}
1 & 2 \\
0 & 1
\end{array}\right)\left(\begin{array}{l}
t \\
x
\end{array}\right)
$$

This diffeomorphism is the $T^{2}$-transformation of the torus. From (2.3) we see that the transformed metric is given by

$$
g^{T^{2}}: \quad d s^{2}=(d t+(a+2) d x)^{2}+d x^{2} .
$$


Thus under $T^{2}$ transformation of the torus we have $a(x) \rightarrow a(x)+2$. This large diffeomorphism of the torus will be the focus of our attention.

Consider the partition function of the theory obtained by integrating out the fermions defined as

$$
Z[g]=\int \mathcal{D} \psi \mathcal{D} \bar{\psi} \exp (-S(\psi, \bar{\psi}, g))
$$

If the theory has a global anomaly, the partition function picks up a phase under the $T^{2}$ transformation [25] which is given by

$$
Z\left[g^{T^{2}}\right]=\exp \left(-i \pi \eta_{1 / 2}\right) Z[g]
$$

The $\eta_{1 / 2}$ invariant is defined as following. Consider the 3-dimensional manifold $\Sigma$ which maps the metric $g$ to $g^{T^{2}}$ through a coordinate $y$. This 3-dimensional manifold is called the mapping torus. The metric is given by

$$
d s_{\Sigma}^{2}=d y^{2}+[d t+(a+2 y) d x]^{2}+d x^{2} .
$$

This metric interpolates between the metric $g$ and $g^{T^{2}}$ as $y$ is dialed from 0 to 1 . Further more from the metric it is clear that we have the identifications

$$
(t, x, y) \sim(t-2 x, x, y+1) .
$$

Thus the torus at $y=0$ is identified with its image at $g^{T^{2}}$. We will choose anti-periodic boundary conditions for the fermions along the time circle $t$ which will eventually be the thermal circle. Then $\eta_{1 / 2}$ is obtained by solving the Dirac equation in $\Sigma$ the 3 dimensional manifold with this boundary condition that $y=0$ and $y=1$ are glued together. Let $\lambda$ denote the eigen value of the Dirac operator

$$
\not D_{1 / 2} \psi=\lambda_{1 / 2} \psi \text {. }
$$

Note that $\psi$ is a Dirac fermion in $\Sigma$. Then the $\eta_{1 / 2}$ invariant is defined by

$$
\eta_{1 / 2}=\sum_{\lambda_{1 / 2}} \operatorname{sign}(\lambda)
$$

The subscript $1 / 2$ in these definitions refer to the fact that we are dealing with the spin- $1 / 2$ fermions.

Evaluating the coefficient $\eta_{1 / 2}$ by solving the Dirac equation on $\Sigma$ is not easy because of the boundary conditions. For the situation in which $\Sigma$ arises as a boundary of a manifold $B$ we can appeal to the Atiyah-Patodi-Singer index theorem to obtain $\eta_{1 / 2}$. Let $B$ be a 4-dimensional manifold such that $\partial B=\Sigma$. The metric on this manifold is given by

$$
d s_{B}^{2}=d r^{2}+d y^{2}+f(r)^{2}[d t+(a+2 y) d x]^{2}+d x^{2} .
$$

Here $r$ takes values from 0 to $1 . f(r)$ is a filling function which has the property

$$
\lim _{r \rightarrow 0} f(r)=r .
$$


Note the above limiting behaviour of $f(r)$ together with the fact that that $t$ is periodic with period $2 \pi$ ensures that this metric does not have a conical singularity at $r=0$. At the boundary $r=1$, the metric reduces to that of $\Sigma$. This metric is essentially that of the solid mapping torus obtained by filling up the $t$ circle. The APS theorem then states that the index of the Dirac operator on $B$ is related to the geometric properties of $B$ by

$$
\operatorname{index}\left(\not D_{1 / 2}\right)(B)=\frac{1}{24 \times 8 \pi^{2}} \int_{B} \operatorname{Tr}(R \wedge R)+\mathcal{I}_{\partial B=\Sigma}-\frac{1}{2} \eta_{1 / 2}
$$

Here $R$ is the curvature 2-form on $B$ and $\mathcal{I}_{\Sigma}$ is an integral over the boundary of $B$ which will be explained in detail in the subsequent section. At present it is sufficient to mention that $\mathcal{I}_{\Sigma}$ are corrections to the APS index theorem for manifolds with boundaries. Essentially the APS theorem offers a geometric means to evaluate $\eta_{1 / 2}$.

Now that one has $\eta_{1 / 2}$ we can go back and write down an effective action which reproduces the change in (2.6). Let

$$
Z[g]=e^{-S_{\mathrm{eff}}}
$$

The metric in (2.2) has an isometry under $t \rightarrow t+\epsilon$. Assuming a gap in the spectrum due to the thermal boundary conditions in the $t$-direction we expect the the low lying effective action to be independent of $t$ and will be a functional of only $a$. An effective action which reproduces the change in (2.6) is given by

$$
S \text { eff }=\frac{i \eta_{1 / 2}}{4} \int a(x) d x
$$

Note that under the $T^{2}$ transformation $a(x) \rightarrow a(x)+2$. This will ensure that we obtain the change given in (2.6) for the partition function. Recall that the integral over $x$ runs from 0 to $2 \pi$. It is clear that this method determines the coefficient in the effective action upto an integer. That is if $\eta_{1 / 2}$ is shifted by an even integer we would still be able to satisfy (2.6).

We will implement this method systematically for chiral fermions, gravitinos and self dual tensors in $d=2,6$. Our goal is to use the effective action to evaluate the parity odd transport and compare them with perturbative calculations done in [16]. To do this we need to introduce further scalings in the metric given in $(2.2)$ so that periodicities in $t$ become $\beta$ the inverse temperature. We must also verify that it is consistent for fermions to satisfy thermal boundary conditions in the geometry we are evaluating the $\eta$-invariant. The periodicity in $x$ should also be scaled to $L$. We will then have to take the $L \rightarrow \infty$ limit. Finally the result for the transport correlator should be analytically continued to Minkowski signature. We will implement all these steps in detail in the subsequent sections.

\section{Global anomalies and transport in $d=2$}

Before we proceed to use the APS theorem to evaluate the $\eta$ invariant for the geometry $\Sigma$ given in (2.7) we first illustrate the simple fact that the partition function of a theory defined on a 2-torus which contains Weyl fermions picks up phase under the $T$ symmetry 
of the torus. Consider free Weyl fermions on a torus with modular parameter $\tau=i \beta / L$. The modular parameter is the ratio of the lengths along the time and the spatial direction of the torus. Let the fermions obey the following boundary conditions on this torus

$$
\begin{array}{ll}
(A, A): \psi(z+1)=-\psi(z), & \psi(z+\tau)=-\psi(z), \\
(P, A): \psi(z+1)=\psi(z), & \psi(z+\tau)=-\psi(z) .
\end{array}
$$

Both these boundary conditions result in thermal partition functions. For free Weyl fermions, these are easily evaluated and we obtain

$$
Z_{A A}(\tau)=\frac{\theta_{3}(\tau)}{\eta(\tau)}, \quad Z_{P A}(\tau)=\frac{\theta_{2}(\tau)}{\eta(\tau)} .
$$

The properties of these partition function under $T$ and $S$ are given by

$$
\begin{array}{ll}
T^{2}: Z_{A A}(\tau+2)=e^{-i \frac{\pi}{6}} Z_{A A}(\tau+2), & S: Z_{A A}\left(-\frac{1}{\tau}\right)=Z_{A A}(\tau), \\
T: Z_{P A}(\tau+1)=e^{+i \frac{\pi}{3}} Z_{P A}(\tau), & S: Z_{P A}\left(-\frac{1}{\tau}\right)=Z_{A P}(\tau)=\frac{\theta_{4}(\tau)}{\eta(\tau)} .
\end{array}
$$

Note that it is only the partition function $Z_{A A}$ which is modular invariant. This partition function has anti-periodic boundary conditions on both the circles. It returns to itself after a $T^{2}: \tau \rightarrow \tau+2$ with a phase $e^{-i \frac{\pi}{6}}$. We will see that the method of evaluating the phase picked up by the $T$ diffeomorphism of the torus using the $\eta$ invariant chooses this boundary conditions. We then will decompactify the theory along the spatial $x$ direction and write down an effective action which ensures that the partition function picks up that required phase.

Let us now determine the change in the partition function by computing the $\eta$ invariant of the $T^{2}$ transformation. Let the change in the partition function for a theory containing Weyl fermions on the 2-torus under the $T^{2}$ transformation be given by

$$
Z\left[g^{T^{2}}\right]=e^{-i \pi \eta_{1 / 2}} Z[g] .
$$

The APS index theorem relates the $\eta$ invariant to the following geometric quantity on the manifold $B$.

$$
\text { Ind }\left(\not D_{\frac{1}{2}}\right)=\frac{1}{24 \times 8 \pi^{2}} \int_{B} \operatorname{Tr}(R \wedge R)-\frac{1}{24 \times 8 \pi^{2}} \int_{\Sigma} \operatorname{Tr}(\theta \wedge R)-\frac{\eta_{\frac{1}{2}}}{2} \text {. }
$$

We have used the expression for the index theorem in [26] which results in opposite sign for $\eta$ compared to that in [25], however this is taken care of by introducing the negative sign for the phase shift in (3.4) . Let us recall the metric on $B$ is given by

$$
d s_{B}^{2}=d r^{2}+d y^{2}+f(r)^{2}(d t+[a(x)+2 y] d x)^{2}+d x^{2} .
$$

In (3.5), $\theta_{\mu b}^{a}$ is the second fundamental form defined as the difference of spin connection $\omega_{\mu b}^{a}$ derived from the metric (3.6) and the spin connection derived from the product metric at the boundary which is given by

$$
d s_{P}^{2}=d r^{2}+d y^{2}+f(1)^{2}(d t+[a(x)+2 y] d x)^{2}+d x^{2} .
$$


Then

$$
\theta_{\mu b}^{a}=\omega_{\mu b}^{a}(B)-\omega_{\mu b}^{a}(B) .
$$

Note that if the manifold $B$ does not have any boundary (3.5) reduces to the usual index theorem one is familiar with.

Before we proceed to evaluate the integrals in the r.h.s. of (3.5) we will discuss the boundary conditions on the fermions. To begin, the boundary conditions in the $t$ direction are anti-periodic due to the topology of $B$. Note that the $(r, t)$ plane has the topology of a disc, since the $t$-circle is filled. Therefore, the fermions obey anti-periodic boundary conditions along the $t$-circle. This is because in the $(r, t)$ plane $t \rightarrow t+2 \pi$ is just a rotation. This is identical to the argument by which the fermions in $A d S_{3}$ obey antiperiodic boundary conditions along the angular directions. Constant time slices in $A d S_{3}$ have the topology of a disc. ${ }^{2}$ The Dirac fermion in $\Sigma$ is periodic in $y$ under $y \rightarrow y+1$ as the metric $g$ is identified with $g^{T^{2}}$ under this shift. $y$ parametrises the direction along with the torus is mapped on to itself. This implies that we must have anti-periodic boundary conditions in $x$ because if there are 2 or more directions in which fermions have periodic boundary conditions, the partition function vanishes due to the presence of of fermionic zero modes. Thus the partition function is evaluated with $(A, A)$ boundary conditions in the $(x, t)$ directions.

We now evaluate the integrals on the l.h.s. of (3.5). Evaluating the curvature components of the metric we obtain

$$
\begin{aligned}
\int_{B} \operatorname{Tr}(R \wedge R) & =-8 \int d y d r d x d t f^{\prime}(r)\left(f^{\prime \prime}(r)+f(r)^{3}\right) \\
& =2(2 \pi)^{2}\left[2\left(f^{\prime}(0)\right)^{2}-2\left(f^{\prime}(1)\right)^{2}+(f(0))^{4}-f(1)^{4}\right] .
\end{aligned}
$$

In evaluating this trace we choose the orientation of the coordinates such that the epsilon tensor is given by $\epsilon^{t x y r}=1$. Using (2.12) we have $f(0)=0, f^{\prime}(0)=1$. Substituting these values we obtain

$$
\frac{1}{24 \times 8 \pi^{2}} \int_{B} \operatorname{Tr}(R \wedge R)=\frac{1}{12}-\frac{\left(f^{\prime}(1)\right)^{2}}{12}-\frac{f(1)^{4}}{24} .
$$

Let us evaluate the boundary term

$$
\frac{1}{24 \times 8 \pi^{2}} \int_{\Sigma} \operatorname{Tr}(\theta \wedge R)=-\frac{\left(f^{\prime}(1)\right)^{2}}{12} .
$$

Thus putting together the integrals on the l.h.s. of the index theorem (3.5) we obtain

$$
\frac{1}{24 \times 8 \pi^{2}}\left(\int_{B} \operatorname{Tr}(R \wedge R)-\int_{\Sigma} \operatorname{Tr}(\theta \wedge R)\right)=\frac{1}{12}-\frac{f(1)^{4}}{24} .
$$

Substituting in (3.5) we obtain

$$
\eta_{\frac{1}{2}}=\frac{1}{6}-\frac{f^{4}(1)}{12}+2 \operatorname{Ind}\left(\not D_{\frac{1}{2}}\right) .
$$

\footnotetext{
${ }^{2}$ See [27] below equation (2.1).
} 
However this equation indicates that $\eta_{1 / 2}$ depends on the filling function $f$ which clearly is not true since $\eta$ is a topological invariant. The reason is because the theory of Weyl fermions contains a perturbative anomaly which results in the following gravitational Chern-Simons term on $\Sigma$

$$
\frac{1}{12 \times 8 \pi^{2}} \int_{\Sigma} \omega \wedge d \omega+\frac{2}{3} \omega \wedge \omega \wedge \omega=-\frac{f^{4}(1)}{12} .
$$

Here $\omega$ is the spin connection in the bulk $B$ but evaluated at the boundary. The original applications of the $\eta$-invariant by [25] involved theories which were free of perturbative anomalies, the anomalies were canceled by the Green-Schwarz mechanism. Here we isolate the topological invariant $\eta_{1 / 2}$ by subtracting the contribution of the gravitational ChernSimons term [20]. The manifold $B$ has the topology of a solid torus, the index the Dirac operator in (3.13) is an integer. The reason is that the the $\eta$-invariant is defined to take into account all the terms mod 2 when the manifold $B$ has a boundary. Therefore this term contributes to a trivial phase shift of the partition function under the $T$ diffeomorphism. Taking all this into account we obtain

$$
\eta_{\frac{1}{2}}=\frac{1}{6}
$$

Thus the phase picked up by the $T^{2}$ transformation is given by

$$
Z\left[g^{T^{2}}\right]=e^{-i \pi \eta_{1 / 2}} Z[g]=e^{-i \frac{\pi}{6}} Z[g] .
$$

This is precisely the phase picked up the $T^{2}$ transformation for fermions with the $(A, A)$ boundary conditions which we evaluated by the direct calculation in (3.3).

\subsection{Fermions}

To obtain the thermal effective action we first need to decompactify the spatial direction. Note that so far we have worked in dimensionless units for the metric say in (2.2). We first introduce dimensions by rescaling the co-ordinates and the metric as

$$
\tilde{x}=\frac{L x}{2 \pi}, \quad \tilde{t}=\frac{\beta t}{2 \pi}, \quad \tilde{d} s^{2}=\frac{\beta^{2}}{(2 \pi)^{2}} d s^{2} .
$$

Then the metric in (2.2) becomes

$$
d \tilde{s}^{2}=(d \tilde{t}+\tilde{a}(\tilde{x}) d \tilde{x})^{2}+d \tilde{x}^{2},
$$

where $\tilde{a}(\tilde{x})$ is defined as

$$
\tilde{a}(\tilde{x})=\frac{\beta}{L} a(x) .
$$

Note now the periodicities $\tilde{x}$ are $\beta$ and $L$ respectively. Now under the $T^{2}$ transformation we have

$$
T:(\tilde{t}, \tilde{x}) \rightarrow\left(\tilde{t}+\frac{2 \beta \tilde{x}}{L}, \tilde{x}\right), \quad \tilde{a} \rightarrow \tilde{a}+\frac{2 \beta}{L} .
$$

We now decompactify the $\tilde{x}$ direction by taking $L$ to be large. The modes in the $x$ direction then become a continuum, but we expect the effective action to still retain the shift by 
the phase $e^{-i \frac{\pi}{6}}$ under the $T^{2}$ diffeomorphisms. The metric in (2.2) is flat, there is no background curvature, therefore the effective action can only depend on $\tilde{a}$. An action which satisfies the required condition of the phase shift is given by

$$
S_{\mathrm{eff}}=\frac{i \pi}{12 \beta} \int \tilde{a}(\tilde{x}) d \tilde{x} \quad Z[g]=e^{-S_{\mathrm{eff}}}
$$

Writing this partition function in momentum space we obtain

$$
Z=\exp \left(-\frac{i \pi}{12} \hat{a}(0)\right)
$$

Here $\hat{a}(0)$ is the Fourier transform at $k=0 .{ }^{3}$ We can now obtain the one point function of the stress tensor $\left\langle T^{\tilde{\tau} \tilde{x}}\right\rangle$. Note that since $\hat{a}$ is the $\tilde{g}_{\tilde{t} \tilde{x}}$ component, the one point function of the stress tensor by ${ }^{4}$

$$
\left\langle T^{\tilde{t} \tilde{x}}(p)\right\rangle=\frac{1}{\sqrt{g}} \frac{\delta \ln Z}{\delta g^{\tilde{t} \tilde{x}}}=\frac{\delta \ln Z}{\delta \hat{a}(p)} .
$$

Evaluating this for the partition function given in (3.22) we obtain

$$
\begin{aligned}
\left\langle T^{\tilde{t} \tilde{x}}(p)\right\rangle & =-\frac{i \pi}{\beta 12} 2 \pi \delta(p), \\
& =-\frac{i \pi}{\beta^{2} 12} 2 \pi \beta \delta(p) .
\end{aligned}
$$

Note that due the definition of the Fourier transform functional differentiation in Fourier space picks up a factor $2 \pi \delta(p)$. We can now go over to Minkowski space by analytical continuation of $t^{\prime}=-i \tilde{t}$. This results in

$$
\begin{aligned}
\left\langle T^{t^{\prime} \tilde{x}^{\prime}}(p)\right\rangle & =\frac{\left\langle T^{\tilde{\tilde{x}} \tilde{x}}(p)\right\rangle}{i}, \\
& =-\frac{\pi}{\beta^{2} 12} 2 \pi \beta \delta(p) .
\end{aligned}
$$

The transport coefficient $\lambda^{(2)}$ which occurs in the constitutive relation for the stress tensor is obtained by evaluating the one point function $-\left\langle T^{t^{\prime} \tilde{x}^{\prime}}(p)\right\rangle$ and then stripping out the $2 \pi \beta \delta(0)$ which occurs in the overall momentum conservation of the correlators in the Kubo formula, see [16] for a discussion. We therefore get

$$
\lambda^{(2)}=\frac{\pi}{12 \beta^{2}}
$$

This coincides with the expression obtained using perturbative calculations in [16] as well as the result using the replacement rule [17].

\footnotetext{
${ }^{3}$ The Fourier transform is defined by $\tilde{a}(x)=\int \frac{d k}{2 \pi} \hat{a}(k) e^{-i k x}$.

${ }^{4}$ This definition of stress tensor is in accordance with [6] for the mostly positive signature.
} 


\subsection{Chiral bosons}

The chiral boson or the self dual tensor in $d=2$ is dual to the Weyl fermion by bosonization. Therefore we expect the same result for the transport coefficient. Lets verify this by evaluating the $\eta_{S}$ for the self dual tensor in $d=2$. The $\eta$ invariant for the self dual tensor can be determined using the APS index formula for self dual tensors. To be general and also relate it to the expressions in [25] we quote the result for arbitrary dimensions.

$$
\frac{\sigma(B)}{8}=\frac{1}{8} L(R)-\mathcal{I}_{\Sigma}(R)+\frac{\eta_{S}}{2} .
$$

Here $L$ is the Hirzebruch polynomial constructed out of the curvature tensor, $\sigma$ the Hirzebruch signature of $B . \mathcal{I}$ is a boundary term which will be defined later. Note that our definition of $\eta_{S}$ is $1 / 4$ the definition used in [25]. The change in the partition function is given by $Z\left[g^{T}\right]=e^{-i \pi \eta_{S}} Z[g]$. Recently the expression in (3.27) has been refined by [28, 29]. The term $\frac{\sigma(B)}{8}$ on the l.h.s. of the equation in (3.27) is replaced by a $\lambda \wedge \lambda$ where $\lambda$ is a $2 k+2$ form for a self dual field in $4 k+2$ dimensions. ${ }^{5}$ Below we will carry our arguments for the version of the index theorem for self dual tensors in [25] as well as provide the arguments for the refined formula for the $\eta$ invariant given in [28]. Let us now substitute the appropriate polynomials for $B$ of dimension 4 in the APS index theorem. We obtain

$$
\frac{\sigma(B)}{8}=-\frac{1}{24 \times 8 \pi^{2}} \int_{B} \operatorname{Tr}(R \wedge R)+\frac{1}{24 \times 8 \pi^{2}} \int_{\Sigma} \operatorname{Tr}(\theta \wedge R)+\frac{\eta_{S}}{2} .
$$

The evaluation of the integrals proceeds exactly as in the case of the fermions. We obtain the relation

$$
\eta_{S}=\frac{1}{6}-\frac{f^{4}(1)}{12}+\frac{\sigma(B)}{4} .
$$

Note that again there is a contribution to $\eta_{S}$ which is removed by subtracting the gravitational Chern-Simons term in (3.14). We now use the fact that for a solid torus $B$ the Hirzebruch signature is a multiple of 8 , again this is because the $\eta$ invariant is defined to take in account of all terms mod 2. For the refined global anomaly formula [28], it is in fact not necessary to make assumptions regarding the Hirzebruch signature of $B$. The term $\sigma(B) / 8$ is replaced by $\lambda \wedge \lambda$ where $\lambda$ is a 2-form. Since in $d=2$ the manifold is a spin manifold we can take the manifold $B$ to have a spin structure compatible with $T^{2}$. In this situation one can show that $\lambda$ can be chosen to vanish. ${ }^{6}$ Therefore to mod 2 we write

$$
\eta_{S}=\frac{1}{6}
$$

This result is identical to that obtained in (3.15) for Weyl fermions. Therefore the result for the transport coefficient $\lambda^{(2)}$ in theories with a single self dual tensor is identical to that of a single Weyl fermion.

\footnotetext{
${ }^{5}$ We thank Samuel Monnier for bringing the references $[28,29]$ to our attention and for explaining the refined formula for the $\eta$ invariant to us. See section 4.3 of [28] for the index theorem without gauge fields and [29] for the index theorem with gauge fields.

${ }^{6}$ We thank Samuel Monnier for this explanation.
} 


\subsection{Gravitinos}

Though there are no physical gravitinos in $d=2$, we can study the 'gravitino like' theory. The gravitino action consists essentially of the spin $3 / 2$ operator acting on the gravitino, the ghosts in the gauge fixing procedure are taken into account by subtracting the contribution of a Weyl fermion Transport coefficient for this theory is evaluated perturbatively in [16]. The results are inconsistent with that obtained using the argument involving the consistency of the Euclidean vacuum. We will show the method of global anomaly matching is consistent with the perturbative evaluation in [16] to mod 2.

The index theorem for gravitinos on closed manifolds is given by $[21,30]$

$$
\operatorname{Index}\left(\not D_{3 / 2}(B)\right)=\int_{B} \hat{A}(B)\left(\operatorname{Tr} e^{i R / 2 \pi}-1\right) .
$$

Expanding the curvature polynomials $\hat{A}(B)$ for the case when the manifold is of 4 dimensions we obtain

$$
\text { Ind }\left(\not D_{\frac{3}{2}}(B)\right)=-\frac{23}{24 \times 8 \pi^{2}} \int_{B} \operatorname{Tr}(R \wedge R) .
$$

Therefore following [25], ${ }^{7}$ the index theorem for manifolds with boundary is given by

$$
\text { Ind }\left(\not D_{\frac{3}{2}}(B)\right)=-\frac{23}{24 \times 8 \pi^{2}} \int_{B} \operatorname{Tr}(R \wedge R)+\frac{23}{24 \times 8 \pi^{2}} \int_{\Sigma} \operatorname{Tr}(\theta \wedge R)-\frac{\eta_{\frac{3}{2}}}{2} \text {. }
$$

Again our definition of $\eta_{3 / 2}$ is of opposite sign compared to that in [25] since in our notation the change in the effective action is given by $Z\left[g^{T}\right] \rightarrow e^{-i \eta_{3 / 2}} Z[g]$. The integrals are identical to the case of the Weyl fermions and proceeding From (3.5), we see that the only difference between the index theorem for gravitinos is the pre factor in front of $\operatorname{Tr}(R \wedge R)$ and $\operatorname{Tr}(\theta \wedge R)$. Proceeding similarly as before we obtain

$$
\eta_{3 / 2}=\frac{-23}{6}-\frac{23 f^{4}(1)}{12}+2 \operatorname{Ind}\left(\not D_{\frac{3}{2}}(B)\right) .
$$

Again the dependence on the filling function $f(1)$ can be removed by adding a ChernSimons term. The index of the spin $3 / 2$ operator on $B$ is an integer since the manifold $B$ has the topology of the solid torus. The $\eta$ invariant takes into account of all terms mod 2 . Therefore we obtain that the $\eta$ invariant for gravitinos to be

$$
\begin{aligned}
\eta_{3 / 2} & =\frac{-23}{6} \bmod 2, \\
& =\frac{1}{6} \bmod 2 .
\end{aligned}
$$

We see that upto mod 2 the $\eta$ invariant for Weyl gravitinos is identical to that of the Weyl fermions. This result is consistent with the direct perturbative calculations of the transport coefficients for gravitinos done in [16]. The perturbative calculations also show that $\lambda^{(2)}$ for gravitinos is identical to that of Weyl fermions in $d=2$. Therefore we conclude that in $d=2$, the transport coefficient for gravitinos obtained from matching global anomalies is consistent with perturbative calculations to mod 2 .

\footnotetext{
${ }^{7}$ In [25] the index theorem for gravitinos is written as the difference of curvature polynomial appropriate for only the spin $3 / 2$ field and the curvature polynomial for fermions. We have combined the two polynomials, so that the end result is that for the 'physical' gravitino.
} 


\section{Global anomalies and transport in $d=6$}

In $d=6$ we start with the following metric $g$ on $\hat{T}^{6}$.

$$
d s^{2}=\left(d t+a_{1}(a) d a+a_{2}(b) d z+a_{3}(y) d x\right)^{2}+d x^{2}+d z^{2}+d a^{2}+d b^{2}+d y^{2} .
$$

Note that now the co-ordinate $a$ will play the role of $x$ in the previous section, this convention has been chosen so that it agrees with that in [16]. The co-ordinates satisfy the periodicity

$$
\begin{aligned}
t & \sim t+2 \pi, & a & \sim a+2 \pi, & & b \sim b+2 \pi, \\
x & \sim x+2 \pi, & y & \sim y+2 \pi, & & z \sim z+2 \pi .
\end{aligned}
$$

We choose anti-periodic boundary conditions for the fermions in all the directions along the torus. Consider the $(x, y)$ plane: since we have $(A, A)$ boundary conditions on this plane, an allowed non-trivial field configuration for the metric component $a_{3}$ is given by

$$
a_{3}(y)=2 n \frac{y}{2 \pi}, \quad n \in \mathbb{Z} .
$$

This ensures that the $a_{3} \rightarrow a_{3}+2 n$ under $y \rightarrow y+2 \pi$. Thus we have a $T^{2}$ transformation in the torus along the $(x, y)$ plane. Therefore the boundary conditions $(A, A)$ remain invariant in these directions. Similarly in the $(z, y)$ plane, to preserve the $(A, A)$ boundary conditions in these directions we consider the non-trivial field configuration

$$
a_{2}(b)=2 m \frac{b}{2 \pi}, \quad m \in \mathbb{Z} .
$$

Thus the fields $a_{3}(y)$ and $a_{2}(b)$ have non-trivial windings along the compact direction. The metric component $a_{1}(a)$ will play the role of field $a(x)$ for the $d=4$ case discussed in the previous sections. We wish to consider the metric related to the one in 4.1 by the $T^{2}$ diffeomorphism given by

$$
\begin{aligned}
d s^{2}= & \left(d t+\left(a_{1}(a)+2\right) d a+a_{2}(b) d z+a_{3}(y) d x\right)^{2} \\
& +d x^{2}+d z^{2}+d a^{2}+d b^{2}+d y^{2} .
\end{aligned}
$$

The 7-dimensional mapping torus $\Sigma$ which interpolates between (4.1) and (4.5) is given by

$$
\begin{aligned}
d s_{\Sigma}^{2}= & d u^{2}+\left(d t+\left[a_{1}(a)+2 u\right] d a+a_{2}(b) d z+a_{3}(y) d x\right)^{2} \\
& +d x^{2}+d z^{2}+d a^{2}+d b^{2}+d y^{2} .
\end{aligned}
$$

Here the coordinate $u$ interpolates between the torus (4.1) and the one related to it by $T^{2}$ diffeomorphism in (4.5) as $u$ runs from 0 to 1 . Therefore we have the identifications

$$
(t, a, u, b, z, x, y) \sim(t-2 a, a, u+1, b, z, x, y) .
$$

Filling up the time circle we get,

$$
\begin{aligned}
d s_{B}^{2}= & d r^{2}+d u^{2}+f(r)^{2}\left(d t+\left[a_{1}(a)+2 u\right] d a+a_{2}(b) d z+a_{3}(y) d x\right)^{2} \\
& +d x^{2}+d z^{2}+d a^{2}+d b^{2}+d y^{2}
\end{aligned}
$$


The filling function satisfies the condition

$$
\lim _{r \rightarrow 0} f(r)=r
$$

for the absence of conical singularities. The product metric at the boundary $r=1$ is defined to be,

$$
\begin{aligned}
d s_{P}^{2}= & d r^{2}+d u^{2}+f(1)^{2}\left(d t+\left[a_{1}(a)+2 u\right] d a+a_{2}(b) d z+a_{3}(y) d x\right)^{2} \\
& +d x^{2}+d z^{2}+d a^{2}+d b^{2}+d y^{2}
\end{aligned}
$$

The metric on $B$ given in (4.8) can now be used to evaluate the $\eta$ invariant for the $T^{2}$ diffeomorphism using the APS index theorem.

The index theorem for the Dirac operator on $B$ is given by

$$
\text { Ind } \begin{aligned}
\left(\not D_{\frac{1}{2}}(B)\right)= & \frac{-1}{6 !} \int_{B}\left(\frac{p_{2}(R)}{2}-\frac{7}{8} p_{1}(R)^{2}\right)-\frac{1}{6 ! \times(2 \pi)^{4}} \int_{\Sigma}\left(\frac{1}{8} \operatorname{Tr}(\theta \wedge R \wedge R \wedge R)\right. \\
& \left.-\frac{1}{16} \operatorname{Tr}(\theta \wedge R) \operatorname{Tr}(R \wedge R)+\frac{7}{32} \operatorname{Tr}(\theta \wedge R) \operatorname{Tr}(R \wedge R)\right)-\frac{\eta_{1 / 2}}{2} .
\end{aligned}
$$

where the Pontryagin classes are defined by

$$
\begin{aligned}
& p_{2}(R)=\frac{-1}{(2 \pi)^{4}}\left(\frac{1}{4} \operatorname{Tr}(R \wedge R \wedge R \wedge R)-\frac{1}{8} \operatorname{Tr}(R \wedge R) \operatorname{Tr}(R \wedge R)\right), \\
& p_{1}(R)=\frac{-1}{(2 \pi)^{2}} \frac{\operatorname{Tr}(R \wedge R)}{2},
\end{aligned}
$$

and $\theta$ is the difference of the spin connections of $B$ and the product metric $P$.

$$
\theta_{\mu b}^{a}=\omega_{\mu b}^{a}(B)-\omega_{\mu b}^{a}(P)
$$

Essentially the index theorem in (4.11) is for a closed 8-manifold with the corrections due to the presence of a boundary. These corrections are obtained by replacing a single $R$ in the anomaly polynomial by $\theta[25]$. Once $\eta_{1 / 2}$ is obtained the change in the partition function is given by

$$
Z\left[g^{T^{2}}\right]=e^{-i \eta_{1 / 2}} Z[g] .
$$

We proceed to evaluate the integrals occurring on the r.h.s. of (4.11).

Contribution from the second Pontryagin class. We first show that all the contributions from the second Pontryagin class to the index theorem in (4.11) is identical to a Chern-Simons term on the boundary $\Sigma$. Consider the term involving the second Pontryagin class in (4.11) along with the associated boundary term which is given by

$$
\begin{aligned}
I_{p_{2}}= & \frac{-1}{6 !} \int_{B}\left(\frac{p_{2}(\mathcal{R})}{2}\right)+\frac{-1}{6 ! \times(2 \pi)^{4}} \int_{\Sigma}\left(\frac{1}{8} \operatorname{Tr}(\theta \wedge R \wedge R \wedge R)\right. \\
& \left.-\frac{1}{16} \operatorname{Tr}(\theta \wedge R) \operatorname{Tr}(R \wedge R)\right) .
\end{aligned}
$$


Substituting the definition of the second Pontryagin class we obtain

$$
\begin{aligned}
I_{p_{2}}= & \frac{1}{6 !(2 \pi)^{4}} \int_{B}\left(\frac{1}{8} \operatorname{Tr}(R \wedge R \wedge R \wedge R)-\frac{1}{16} \operatorname{Tr}(R \wedge R) \operatorname{Tr}(R \wedge R)\right) \\
& +\frac{-1}{6 ! \times(2 \pi)^{4}} \int_{\Sigma}\left(\frac{1}{8} \operatorname{Tr}(\theta \wedge R \wedge R \wedge R)-\frac{1}{16} \operatorname{Tr}(\theta \wedge R) \operatorname{Tr}(R \wedge R)\right) .
\end{aligned}
$$

Each of these integrals have been evaluated in (A.6). On substituting the results for the integrals we obtain

$$
\begin{aligned}
I_{p_{2}}= & \frac{-1}{4 \times 6 !} \int d b d y a_{2}^{\prime}(b) a_{3}^{\prime}(y)\left\{3 f(1)^{8}\left[a_{2}^{\prime}(b)^{2}+a_{3}^{\prime}(y)^{2}+a_{2}^{\prime}(b)^{2}\left(1+a_{3}^{\prime}(y)^{2}\right)\right]\right. \\
& \left.+4 f(1)^{4} f^{\prime}(1)^{2}\left(1+a_{2}^{\prime}(b)^{2}+a_{3}^{\prime}(y)^{2}\right)\right\} .
\end{aligned}
$$

Note that in the integral $I_{p_{2}}$, all terms depend on the filling function $f$, therefore we expect the entire contribution not to contribute to the topological $\eta$ invariant. We will now show that the entire contribution of $I_{p_{2}}$ can be be accounted by a Chern-Simons term on the boundary $\Sigma$. Let us define the Chern-Simons form on $\Sigma$

$$
\begin{aligned}
\mathcal{I}_{C S_{1}}= & \frac{1}{(2 \pi)^{4}} \frac{1}{8}\left[\operatorname{Tr}(\omega \wedge d \omega \wedge d \omega \wedge d \omega)+\frac{8}{5} \operatorname{Tr}(d \omega \wedge d \omega \wedge \omega \wedge \omega \wedge \omega)\right. \\
& +\frac{4}{5} \operatorname{Tr}(d \omega \wedge \omega \wedge d \omega \wedge \omega \wedge \omega)+ \\
& +\frac{4}{7} \operatorname{Tr}(\omega \wedge \omega \wedge \omega \wedge \omega \wedge \omega \wedge \omega \wedge \omega)+2 \operatorname{Tr}(d \omega \wedge \omega \wedge \omega \wedge \omega \wedge \omega \wedge \omega) \\
& \left.-\frac{1}{16} \operatorname{Tr}\left(\omega \wedge d \omega+\frac{2}{3} \omega \wedge \omega \wedge \omega\right) \operatorname{Tr}(R \wedge R)\right]
\end{aligned}
$$

It is can be verified that

$$
-\frac{1}{6 !} p_{2}(R)=\frac{1}{6 !} d\left(\mathcal{I}_{C S_{1}}\right) .
$$

We now integrate the Chern-Simons form $\mathcal{I}$ on the boundary $\Sigma$. The integrals of each of the terms occurring in $\mathcal{I}$ is given in (A.13). Putting all the terms together we obtain

$$
\begin{aligned}
I_{C S_{1}}= & \frac{1}{6 !} \int_{\Sigma} \mathcal{I}_{C S_{1}}, \\
= & \frac{-1}{4 \times 6 !} \int d b d y a_{2}^{\prime}(b) a_{3}^{\prime}(y)\left\{3 f(1)^{8}\left[a_{2}^{\prime}(b)^{2}+a_{3}^{\prime}(y)^{2}+a_{2}^{\prime}(b)^{2}\left(1+a_{3}^{\prime}(y)^{2}\right)\right]\right. \\
& \left.+4 f(1)^{4} f^{\prime}(1)^{2}\left(1+a_{2}^{\prime}(b)^{2}+a_{3}^{\prime}(y)^{2}\right)\right\} .
\end{aligned}
$$

In performing this integrals note that the spin connection $\omega, d \omega$ is evaluated at the boundary $r=1$, The curvature forms in the last term of (4.17) is the curvature of the bulk metric evaluated at the boundary.

It is indeed remarkable that the contribution of the second Pontryagin class to the APS index theorem given in (4.16) coincides precisely with the value of the Chern-Simons form in (4.20). Therefore we can completely remove the contribution of the second Pontryagin class by subtracting out the Chern-Simons term (4.20) at the boundary. As in the case of 
$d=2$ studied in the earlier section, this operation ensures that we pick up only the purely topological terms in the $\eta$ invariant. Thus we conclude the the second Pontryagin class does not contribute to the purely topological terms in the $\eta$ invariant and therefore will not contribute to transport. This was observed in the 'replacement rule' $[17,18,24]$ as well as the holographic calculations of [31].

Contributions from square of the first Pontryagin class. On the r.h.s. of the index theorem (4.11) the contributions due to $p_{1}^{2}$ is given by

$$
\begin{aligned}
I_{p_{1}} & =\frac{7}{8 \times 6 !} \int_{X}\left(p_{1}(R)^{2}\right)+\frac{-7}{32 \times 6 ! \times(2 \pi)^{4}} \int_{\Sigma}(\operatorname{Tr}(\theta \wedge R) \operatorname{Tr}(R \wedge R)), \\
& =\frac{7}{32 \times 6 ! \times(2 \pi)^{4}} \int_{X}(\operatorname{Tr}(R \wedge R) \operatorname{Tr}(R \wedge R))-\frac{7}{32 \times 6 ! \times(2 \pi)^{4}} \int_{\Sigma}(\operatorname{Tr}(\theta \wedge R) \operatorname{Tr}(R \wedge R)) .
\end{aligned}
$$

Substituting from (A.6) for the curvature integrals we obtain

$$
\begin{aligned}
I_{p_{1}}= & \frac{-7}{32 \times 6 !} f(1)^{4} a_{2}^{\prime}(b) a_{3}^{\prime}(y)\left\{-16 f^{\prime}(1)^{2}\left(a_{2}^{\prime}(b)^{2}+a_{3}^{\prime}(y)^{2}+1\right)\right. \\
& \left.-f(1)^{4}\left[11 a_{2}^{\prime}(b)^{2}\left(a_{3}^{\prime}(y)^{2}+1\right)+5 a_{2}^{\prime}(b)^{4}+5 a_{3}^{\prime}(y)^{4}+11 a_{3}^{\prime}(y)^{2}+5\right]+48\right\} .
\end{aligned}
$$

Note that this integral contains terms which depend on the filling function $f$ as well as the pure topological term which arises from the last term in (4.22). We will again show that all terms that depend on the filling function can be canceled by a Chern-Simons term evaluate at the boundary $\Sigma$. From the appendix we have the identity

$$
p_{1}^{2}(R)=\frac{1}{4(2 \pi)^{4}} d\left(\operatorname{Tr}\left(\omega \wedge d \omega+\frac{2}{3} \omega \wedge \omega \wedge \omega\right) \operatorname{Tr}(R \wedge R)\right) .
$$

Therefore we consider the Chern-Simons term

$$
I_{C S_{2}}=\frac{7}{32 \times 6 ! \times(2 \pi)^{4}} \int_{\Sigma} \operatorname{Tr}\left(\omega \wedge d \omega+\frac{2}{3} \omega \wedge \omega \wedge \omega \operatorname{Tr}(R \wedge R)\right) .
$$

Substituting for the spin connection at the boundary and the bulk curvature, but evaluated at the boundary we obtain

$$
\begin{aligned}
I_{C S_{2}}= & \frac{-7}{32 \times 6 !} f(1)^{4} a_{2}^{\prime}(b) a_{3}^{\prime}(y)\left\{-16 f^{\prime}(1)^{2}\left(a_{2}^{\prime}(b)^{2}+a_{3}^{\prime}(y)^{2}+1\right)\right. \\
& \left.-f(1)^{4}\left[11 a_{2}^{\prime}(b)^{2}\left(a_{3}^{\prime}(y)^{2}+1\right)+5 a_{2}^{\prime}(b)^{4}+5 a_{3}^{\prime}(y)^{4}+11 a_{3}^{\prime}(y)^{2}+5\right]\right\} .
\end{aligned}
$$

Note the absence of the last term of (4.22) in (4.25).

Now using the results in (4.16), (4.20), (4.22) and (4.25) we can write the index theorem in $(4.11)$ as

$$
\frac{\eta_{1 / 2}}{2}=-\frac{7}{480(2 \pi)^{2}} \int d b d y d a d z a_{2}^{\prime}(b) a_{3}^{\prime}(y)+I_{C S 1}+I_{C S 2}-\operatorname{Ind}\left(\not D_{1 / 2}(B)\right) .
$$

Again note that removing the Chern-Simons terms we obtain a purely topological $\eta$ invariant, also the index of the Dirac operator is an integer. Therefore we obtain that the 
shift of the phase in the path integral under the $T^{2}$ transformation, $a_{1}(a) \rightarrow a_{1}(a)+2$ is given by

$$
\eta_{1 / 2}=-\frac{7}{240(2 \pi)^{2}} \int d b d y d x d z a_{2}^{\prime}(b) a_{3}^{\prime}(y) \quad \bmod 2 .
$$

Due to the quantization conditions (4.3) and (4.4) we obtain

$$
\eta_{1 / 2}=-\frac{7 n m}{60} \quad \bmod 2
$$

An effective action which reproduces this phase shift is given by

$$
S_{\text {eff }}=-\frac{i 7 \pi}{480(2 \pi)^{3}} \int d a d b d x d y d z a_{1}(a) a_{2}^{\prime}(b) a_{3}^{\prime}(y) .
$$

Note that under $a_{1} \rightarrow a_{1}+2$ the phase shift from this effective action is given by $e^{-i \pi \eta_{1 / 2}}$. This effective action can formally be written as a Chern-Simons form by introducing the graviphoton field $A=A_{\mu} d x^{\mu}$ as

$$
S_{\text {eff }}=-\frac{i 7 \pi}{960(2 \pi)^{3}} \int A \wedge d A \wedge d A
$$

\subsection{Fermions}

To take the decompactification limit and to introduce the temperature we resale the coordinates as

$$
\begin{aligned}
& \tilde{a}=\frac{L_{a} a}{2 \pi}, \\
& \tilde{t}=\frac{\beta t}{2 \pi}, \\
& \tilde{x}=\frac{L_{x} x}{2 \pi}, \\
& \tilde{y}=\frac{L_{y} a}{2 \pi}, \\
& \tilde{z}=\frac{L_{z} a}{2 \pi}, \\
& \tilde{b}=\frac{L_{b} b}{2 \pi} \text {. }
\end{aligned}
$$

After introducing dimensions by rescaling the metric using

$$
\tilde{d} s^{2}=\left(\frac{\beta}{2 \pi}\right)^{2}
$$

the metric in (4.1) becomes

$$
\begin{aligned}
d \tilde{s}^{2}= & \left(d \tilde{t}+\tilde{a}_{1}(\tilde{a}) d \tilde{a}+\tilde{a}_{2}(\tilde{b}) d \tilde{z}+\tilde{a}_{3}(\tilde{y}) d \tilde{x}\right)^{2} \\
& +\left(\frac{\beta}{L_{x}}\right)^{2} d \tilde{x}^{2}+\left(\frac{\beta}{L_{z}}\right)^{2} d \tilde{z}^{2}+\left(\frac{\beta}{L_{a}}\right)^{2} d \tilde{a}^{2}+\left(\frac{\beta}{L_{b}}\right)^{2} d \tilde{b}^{2}+\left(\frac{\beta}{L_{y}}\right)^{2} d \tilde{y}^{2},
\end{aligned}
$$

where $\tilde{a_{1}}, \tilde{a_{2}}, \tilde{a_{3}}$ are defined as,

$$
\tilde{a_{1}}=\frac{\beta}{L_{a}} a_{1}, \quad \tilde{a_{2}}=\frac{\beta}{L_{z}} a_{2}, \quad \tilde{a_{3}}=\frac{\beta}{L_{x}} a_{3} .
$$

After these change of variables, the effective action in (4.29) becomes

$$
S_{\mathrm{eff}}=\frac{-i 7 \pi}{\beta^{3} 480} \int d \tilde{b} d \tilde{y} d \tilde{a} d \tilde{z} d \tilde{x} \tilde{a}_{1}(\tilde{a}) \tilde{a}_{2}^{\prime}(\tilde{b}) \tilde{a}_{3}^{\prime}(\tilde{y}) .
$$


We decompactify the spatial directions and then write the action in Fourier space, we obtain

$$
S_{\text {eff }}=\frac{-i 7 \pi}{\beta^{3} 480} \int \frac{d^{5} p d^{5} k}{\left((2 \pi)^{5}\right)^{2}}\left(i k^{b} i p^{y}\right) \tilde{a}_{1}(-p-k) \tilde{a}_{2}(k) \tilde{a}_{3}(p) .
$$

The transport coefficient $\lambda_{3}^{(6)}$ which is sensitive to the pure gravitational anomaly is defined by the following Kubo formula [16]

$$
\begin{aligned}
\lambda_{3}^{6} & =-\frac{3\left\langle T^{t a}(-p-k) T^{t x}(p) T^{t z}(k)\right\rangle}{2\left(i p^{y}\right)\left(i k^{b}\right)}, \\
& =-\frac{3 i\left\langle T^{\tau a}(-p-k) T^{\tau x}(p) T^{\tau z}(k)\right\rangle}{2\left(i p^{y}\right)\left(i k^{b}\right)}, \\
& =-\frac{3 i}{\left(2 i p^{y}\right)\left(i k^{b}\right)} \frac{\delta^{3} \ln Z}{\delta g_{\tau a} \delta g_{\tau x} \delta g_{\tau z}} .
\end{aligned}
$$

In the second line of the above equation we have analytically continued to Euclidean correlators using $t=-i \tau$. In the last line we have written the correlator in terms of derivatives on the partition function. Using $\ln Z=-S_{\text {eff }}$ and identifying $\delta g_{\tau a}=\delta a_{1}, \delta g_{\tau z}=\delta a_{2}, \delta g_{\tau x}=$ $\delta a_{3}$ we obtain

$$
\begin{aligned}
\lambda_{3}^{6} & =-\frac{3 i}{2\left(i p^{y}\right)\left(i k^{b}\right)} \frac{(i 7 \pi)\left(i p^{y}\right)\left(i k^{b}\right)}{\beta^{4} 480}(2 \pi)^{5} \beta \delta(0), \\
& =\frac{7 \pi}{320 \beta^{4}} \times(2 \pi)^{5} \beta \delta(0) .
\end{aligned}
$$

In the last line we have factored out the terms which are due to the overall momentum conservation. Therefore we obtain

$$
\lambda_{3(1 / 2)}^{6}=\frac{7 \pi}{320 \beta^{4}} .
$$

This result coincides with the one obtained in [16] using perturbation theory at one loop. ${ }^{8}$

\subsection{Gravitinos}

We will now show that the constraints obtained for the thermal effective action for gravitinos using global anomalies is consistent with the result for the transport coefficient $\lambda_{3}^{(6)}$ obtained using perturbation theory in [16]. The APS index theorem for gravitinos is given by

$$
\begin{aligned}
\operatorname{Ind}\left(\not D_{\frac{3}{2}}\right)= & \frac{-1}{6 !} \int_{B}\left(\frac{245 p_{2}(\mathcal{R})}{2}-\frac{275}{8} p_{1}(\mathcal{R})^{2}\right)-\frac{1}{6 ! \times(2 \pi)^{4}} \int_{\Sigma}\left(\frac{245}{8} \operatorname{Tr}(\theta \wedge R \wedge R \wedge R)\right. \\
& \left.-\frac{245}{16} \operatorname{Tr}(\theta \wedge R) \operatorname{Tr}(R \wedge R)+\frac{275}{32} \operatorname{Tr}(\theta \wedge R) \operatorname{Tr}(R \wedge R)\right)-\frac{\eta_{\frac{3}{2}}}{2} .
\end{aligned}
$$

The coefficients in front of the curvature polynomials take care of the subtraction of the ghosts and therefore the result for the $\eta$ is for the 'physical gravitino'.

\footnotetext{
${ }^{8}$ Note the first term in equation (4.39) of [16].
} 
Evaluating the curvature polynomials just as in the spin $1 / 2$ case we obtain

$$
\eta_{3 / 2}=-\frac{1}{(2 \pi)^{2}} \frac{275}{240} \int d b d y d x d z a_{2}^{\prime}(b) a_{3}^{\prime}(y)+\frac{450}{7} I_{C S_{2}}+490 I_{C S_{1}} .
$$

Here we have dropped the contribution of the index of the spin $3 / 2$ operator since it is an integer for the solid torus. Now the pure topological term in $\eta_{3 / 2}$ is extracted by removing the Chern-Simon terms. Finally we also substitute the possible winding configurations given in (4.3) and (4.4) for the graviphoton fields $a_{2}, a_{3}$. This reduces (4.41) to

$$
\begin{aligned}
\eta_{3 / 2} & =-\frac{275}{60} n m, \\
& =-\frac{35}{60} n m-4 n m=-\frac{35}{60} \bmod 2 .
\end{aligned}
$$

Therefore up to mod 2 we can write $\eta_{3 / 2}$ as

$$
\eta_{3 / 2}=-\frac{1}{(2 \pi)^{2}} \frac{35}{480} \int d b d y d x d z a_{2}^{\prime}(b) a_{3}^{\prime}(y) .
$$

The effective action which reproduces this phase shift under $a_{1} \rightarrow a_{1}+2$ is given by

$$
S_{\mathrm{eff}}=-\frac{i 35 \pi}{480(2 \pi)^{3}} \int d b d y d x d a d z a_{1}(a) a_{2}^{\prime}(b) a_{3}^{\prime}(y) .
$$

Note that this is 5 times the result obtained for the Weyl fermion in (4.29). Therefore on decompactifying the spatial directions and extracting out the transport coefficient for the gravitinos we obtain

$$
\lambda_{3(3 / 2)}^{(6)}=\frac{35 \pi}{320 \beta^{4}} .
$$

The above result coincides with that obtained using perturbation theory at one loop [16].

The general pattern seen for the contribution of the gravitino to the transport coefficient in $2 d$ dimensions is that its value is $2 d-1$ times that the result for the Weyl fermion. It is remarkable that mod 2 ambiguity in determining the thermal effective action using global anomalies is consistent with this value of the transport coefficient for the gravitino.

\subsection{Self-dual tensors}

The APS index theorem for self-dual tensors in $d=6$ is given by

$$
\begin{aligned}
\frac{\sigma_{S}(B)}{8}= & \frac{-1}{6 !} \int_{B}\left(\frac{28 p_{2}(R)}{2}-\frac{16}{8} p_{1}(R)^{2}\right)-\frac{1}{6 ! \times(2 \pi)^{4}} \int_{\Sigma}\left(\frac{28}{8} \operatorname{Tr}(\theta \wedge R \wedge R \wedge R)\right. \\
& \left.-\frac{28}{16} \operatorname{Tr}(\theta \wedge R) \operatorname{Tr}(R \wedge R)+\frac{16}{32} \operatorname{Tr}(\theta \wedge R) \operatorname{Tr}(R \wedge R)\right)-\frac{\eta_{A}}{2} .
\end{aligned}
$$

Going through the same steps of evaluating the curvature polynomial and using the fact that the Hirzebruch index for a solid torus vanishes we obtain

$$
\eta_{A}=\frac{-16}{240(2 \pi)^{2}} \int d b d y d a d z a_{2}^{\prime}(b) a_{3}^{\prime}(y)+56 I_{C S 1}+\frac{32}{7} I_{C S 2} .
$$


For the refined global anomaly expression of [28] the term $\sigma(B) / 8$ is replaced by $\lambda \wedge \lambda$ where $\lambda$ is a 4 -form. Now for the solid torus $B$ which is a disc times a torus, $D_{2} \times T^{6}$. The relative cohomology of the disc has a unique generator of degree 2 . This ensures that the intersection pairing in degree 4 of the relative cohomology of $D_{2} \times T^{6}$ vanishes. For this situation we can take $\lambda=0 .{ }^{9}$ For the refined global anomaly formula again there is no need to make an assumption regarding the Hirzebruch index of $B$ and we obtain the same result as in (4.47). Extracting out the topological term by dropping the Chern-Simons contribution we obtain

$$
\eta_{A}=\frac{-16}{240(2 \pi)^{2}} \int d b d y d x d z a_{2}^{\prime}(b) a_{3}^{\prime}(y)
$$

The thermal effective action which reproduces this phase shift under $a_{1} \rightarrow a_{1}+2$ is given by

$$
S_{\mathrm{eff}}=\frac{-i 16 \pi}{480(2 \pi)^{3}} \int d b d y d x d a d z a_{1}(a) a_{2}^{\prime}(b) a_{3}^{\prime}(y) .
$$

Now going through the same steps of decompactifying the spatial directions and extracting out the transport coefficient we obtain the following result for self-dual tensors

$$
\lambda_{3(S)}^{(6)}=\frac{16 \pi}{320 \beta^{4}}=\frac{\pi}{20 \beta^{4}} .
$$

In the next section we will verify this result by an explicit perturbative calculation for the self dual tensors.

\section{Transport for self dual tensors in $d=6$ at one loop}

Self dual tensors in $4 k+2$ dimensions have no Lorentz invariant action though they have Lorentz covariant equations of motion. Pure gravitational anomalies exhibited by these theories were studied in perturbation theory by [21]. They proposed Feynman rules and the propagator for these fields by which gravitational anomalies in these theories were evaluated. In this section we use these rules at finite temperature to evaluate the transport coefficient $\lambda_{3}^{(6)}$ in $d=6$. The field strength of the self dual anti symmetric tensor is defined by

$$
F_{\mu_{1} \mu_{2} \mu_{3}}=\partial_{\mu_{1}} A_{\mu_{2} \mu_{3}}+\text { (cyclic permutations), }
$$

where $A_{\mu_{1} \mu_{2}}$ is the 2 nd rank anti-symmetric gauge potential. The self dual condition in Euclidean space is given by

$$
F^{\mu_{1} \mu_{2} \mu_{3}}=\frac{i}{3 ! \sqrt{g}} \epsilon^{\mu_{1} \mu_{2} \mu_{3} \nu_{1} \nu_{2} \nu_{3}} F_{\nu_{1} \nu_{2} \nu_{3}} \equiv i \tilde{F}^{\mu \nu}
$$

where the orientation is chosen by setting

$$
\epsilon^{\tau a z x y b}=1 .
$$

\footnotetext{
${ }^{9}$ We again thank Samuel Monnier for explaining this to us.
} 
Let us consider the theory of self dual tensors coupled to metric fluctuations. We work in Euclidean space with the signature $\eta^{\mu \nu}=\operatorname{diag}(-1,-1,-1,-1,-1,-1)$ The Kubo formula for the transport coefficient of interest in Euclidean space is given by

$$
\tilde{\lambda}_{3}^{(6)}=-\frac{3}{2} \lim _{p_{b}, k_{y} \rightarrow 0} \frac{\left\langle T^{\tau a}(k+p) T^{\tau x}(-k) T^{\tau z}(-p)\right\rangle}{i p_{b} i k_{y}} .
$$

where $p, k$ are the external momenta. They are chosen such that

$$
p=\left\{0,0,0,0,0, p^{b}\right\}, \quad k=\left\{0,0,0,0, k^{y}, 0\right\} .
$$

The correlator in Minkowski space is related to that in (5.4) by

$$
\tilde{\lambda}_{3}^{(6)}=-i \lambda_{3}^{(6)} .
$$

Note that in (5.4) we have taken all the external frequencies to zero first. The stress tensor for the self dual boson is defined as follows $[21] .{ }^{10}$ First consider

$$
T_{\mu \nu}(F)=-\frac{1}{2} F_{\mu \alpha \beta} F_{\nu}^{\alpha \beta}+\frac{1}{12} g_{\mu \nu} F_{\alpha \beta \gamma} F^{\alpha \beta \gamma} .
$$

Now we impose the self dual condition by considering

$$
T_{\mu \nu}\left(F^{+}\right)=T_{\mu \nu}\left(\frac{1}{2}(F+i \tilde{F})\right) .
$$

The hydrodynamic correlation function in (5.4) includes the following expectation values

$$
\begin{aligned}
\left\langle T^{\mu \alpha} T^{\nu \beta} T^{\rho \sigma}\right\rangle_{E}= & \left\langle T_{f l}^{\mu \alpha} T_{f l}^{\nu \beta} T_{f l}^{\rho \sigma}\right\rangle_{E} \\
& -2\left\langle\frac{\delta T^{\mu \alpha}}{\sqrt{g} \delta g_{\nu \beta}} T^{\rho \sigma}\right\rangle_{E}-2\left\langle\frac{\delta T^{\mu \alpha}}{\sqrt{g} \delta g_{\rho \beta}} T^{\nu \sigma}\right\rangle_{E} \\
& -2\left\langle T^{\mu \alpha} \frac{\delta T^{\nu \beta}}{\sqrt{g} \delta g_{\rho \sigma}}\right\rangle_{E}+4\left\langle\frac{\delta^{2} T^{\mu \alpha}}{\sqrt{g} \delta g_{\nu \alpha} \delta g_{\rho \sigma}}\right\rangle_{E} .
\end{aligned}
$$

All these expectation values are taken in the Euclidean vacuum. The first term on the r.h.s. is the stress tensor evaluated in flat space. All the rest of the terms are contact terms which need to be evaluated carefully. Note that in each of the stress tensor insertions we need to impose the self dual condition by using (5.8).

Before we discuss the contact terms, we will present the propagator to evaluate these correlators. The thermal 2-point function of the gauge invariant fields is given by

$$
\begin{aligned}
S_{B}\left(\omega_{n}, p\right) & =\left\langle F^{\mu_{1} \mu_{2} \mu_{3}}\left(\omega_{n}, p\right) F^{\nu_{1} \nu_{2} \nu_{3}}\left(-\omega_{n^{\prime}},-p_{3}\right)\right\rangle, \\
& =\left(\frac{p^{\mu_{1}} p^{\nu_{1}} g^{\mu_{2} \nu_{2}} g^{\mu_{3} \nu_{3}}}{\omega_{n}^{2}+p^{2}}+\text { Permutations }\right) \beta \delta_{n, n^{\prime}}(2 \pi)^{5} \delta^{5}\left(p-p_{3}\right),
\end{aligned}
$$

\footnotetext{
${ }^{10}$ We have fixed the over all sign in the stress tensor by demanding that it agrees with the 2 dimensional conformal field theory definition of the stress tensor for the chiral boson when applied to $d=2$. The reason the sign differs from that in [21] is due to our choice of mostly negative signature of space time.
} 
where, the frequencies for the bosons in the Euclidean theory are even multiples of $\pi T$ and are given by

$$
\omega_{n}=2 n \pi T, \quad n \in \mathbb{Z} .
$$

Lets now discuss how we proceed to evaluate each of the terms in (5.9). The first term is obtained by evaluating the Wick contractions of the flat space stress tensor written in momentum space. To be explicit we write down the $(\tau x)$ component of the stress tensor

$$
\begin{aligned}
T_{f l}^{\tau x}(-k)= & -\frac{1}{\beta} \Sigma_{\omega_{m}} \int \frac{d^{5} p_{2}}{(2 \pi)^{5}}\left\{F^{\tau a b}\left(-p_{2}-k\right) F^{x a b}\left(p_{2}\right)+F^{\tau a y}\left(-p_{2}-k\right) F^{x a y}\left(p_{2}\right)\right. \\
& +F^{\tau a z}\left(-p_{2}-k\right) F^{x a z}\left(p_{2}\right)+F^{\tau b y}\left(-p_{2}-k\right) F^{x b y}\left(p_{2}\right) \\
& \left.+F^{\tau b z}\left(-p_{2}-k\right) F^{x b z}\left(p_{2}\right)+F^{\tau y z}\left(-p_{2}-k\right) F^{x y z}\left(p_{2}\right)\right\} .
\end{aligned}
$$

Here the dependence on the Matsubara frequency in the integrand is present in $p_{2}$ whose time component is $\omega_{m}$, which we have not been explicit. Note that though we need to impose the self dual projection on the field strength at every insertion of the stress tensor it is sufficient to work with the self dual insertion on one of the insertions of the stress in the Wick contractions [21]. The details of all the Wick contractions are performed in the appendix (B). To evaluate the contact terms in (5.9) we expand the stress tensor in (5.7) by considering only metric fluctuations $h_{\tau x}(k)$ and $h_{\tau z}(p)$. Here we write down an example of the action of the derivative with respect to $h_{\tau z}$ on $T^{\tau x}$

$$
\begin{aligned}
\frac{\delta T^{\tau x}(-k)}{\delta h_{\tau z}(p)}= & -\sum_{\omega_{m}} \int \frac{d^{5} p_{3}}{(2 \pi)^{5}}\left\{F^{z a b}\left(-p_{3}-p-k\right) F^{x a b}\left(p_{3}\right)+F^{z a y}\left(-p_{3}-p-k\right) F^{x a y}\left(p_{3}\right)\right. \\
& \left.+F^{z b y}\left(-p_{3}-p-k\right) F^{x b y}\left(p_{3}\right)\right\}
\end{aligned}
$$

Here again we have suppressed the dependence of the Matsubara frequency in the time component of $p_{3}$. We have to Wick contract the above expression with $T^{\tau a}(k+p)$ on which the self dual projection is inserted. Similar terms are written down for these class of contact terms. In the appendix B, it is shown in detail how all these contact terms yield vanishing contribution to the transport coefficient. Finally we have the last contact term in (5.9) resulting from two derivatives of the metric on the stress tensor. It is shown in the appendix that this term also vanishes. In summary we do not have any contribution from the contact terms.

The analysis of all possible Wick contractions is tedious and has to be done very methodically. This is also performed in detail in the appendix B. After the Wick contractions there are angular integrals over the internal momenta to be performed. We perform these integrals using the method developed in [16]. Essentially we take the zero external frequency limit first and then take the external momenta to zero before the integration. The integrands then simplify considerably and the integrals are easily performed. Finally all the finite terms resulting from the Wick contractions in the zero momentum limit of the correlator (5.4) are given in (B.1). The end result of this long calculation yields the following result for the transport coefficient

$$
\lambda_{3(S)}^{(6)}=\frac{\pi T^{4}}{20} .
$$

This agrees with (4.50), the result obtained using global anomaly matching. 


\section{Conclusions}

We have used the method of global anomaly matching put forward in [20] for theories with chiral gravitinos and self dual tensors to determine thermal effective actions and therefore parity odd transport coefficients. For the case of gravitinos, we obtain results for transport coefficients which are consistent with perturbative calculations of [16] up to mod 2. Our analysis in $d=6$ shows that the second Pontryagin class does not contribute to the topological $\eta$ invariant and therefore does not contribute to transport. This provides a topological explanation for this observation in the replacement rule of [22]. Finally we have evaluated the transport coefficients of self dual tensors in $d=6$ using the Feynman rules put forward in [21]. As far as we are aware this is the first instance where the Feynman rules proposed by [21] for the self dual tensor has been used at finite temperature. It is indeed satisfying that the result agrees with the expectation from global anomaly matching as well as the 'replacement rule' of [22].

From the study in this paper it is clear that these transport coefficients are not perturbatively renormalized ${ }^{11}$ since they are related to global anomalies up to mod 2 . However it will be interesting to figure out the reason which can invoked to fix the mod 2 ambiguity in the method of global anomalies. One thing we have roughly checked that is this ambiguity persists also for gravitinos in $d=10$. It is also of interest to note that it is more easy to determined the $\eta$ invariant using the replacement rule of [22] that the direct calculation of various curvature invariants. There are no holographic checks for the transport coefficients of self dual tensors and gravitinos since these fields usually do not occur as dynamic fields in boundary theories. But, it will be interesting to devise other situations where contribution to transport coefficients from these fields can be checked. Lastly, we claim that the $\eta$ invariant calculated by using the index theorem can be verified by the computation of various correlators in weak coupling regime. This provides an easier alternative way to compute the $\eta$-invariant upto a factor of mod 2. In appendix $\mathrm{C}$ we have evaluated the $\eta$ invariant for the $T^{2}$ transformation for various chiral matter in $d=10$ using the replacement rule of $[22] .{ }^{12}$ It is easy to repeat this exercise for arbitrary even dimensions.

\section{Acknowledgments}

We thank R. Loganayagam for numerous discussions and suggestions which helped clarify our understanding of the issues related to this project. We thank Samuel Monnier for very useful correspondence which helped us update our understanding of the global anomaly formula for self dual tensors. He also helped us in understanding how the refined global anomaly formula applies for the $T$-diffeomorphisim dealt with in this paper. We also thank Kallol Sen for helping us automate performing Wick contractions in Mathematica.

\footnotetext{
${ }^{11}$ Recently non-renormalization of anomalous transport coefficients was studied holographically in [32].

${ }^{12}$ We thank R. Loganayagam for suggesting us to carry out this exercise.
} 


\section{A Curvature integrals and Chern-Simons terms}

It is useful to list both the vielbeins used in evaluating the difference between the spin connections of the bulk and the product metric which is defined by

$$
\theta=\omega_{\mu b}^{a}(B)-\omega_{\mu b}^{a}(P)
$$

2d vielbeins. The vielbein which was used for evaluating the spin connection $\omega_{\mu b}^{a}(B)$ for the metric in (3.6) are given by

$$
\begin{aligned}
& e_{r}^{\hat{r}}(B)=1, \quad e_{y}^{\hat{y}}(B)=1, \\
& e_{t}^{\hat{t}}(B)=f(r), \quad e_{x}^{\hat{t}}(B)=f(r)(a(x)+2 y), \quad e_{x}^{\hat{x}}(B)=1 .
\end{aligned}
$$

The vielbein used of evaluating the spin connection $\omega_{\mu b}^{a}(P)$ for the metric in (3.7) are given by

$$
\begin{aligned}
& e_{r}^{\hat{r}}(P)=1, \quad e_{y}^{\hat{y}}(P)=1, \\
& e_{t}^{\hat{t}}(P)=f(1), \quad e_{x}^{\hat{t}}(P)=f(1)(a(x)+2 y), \quad e_{x}^{\hat{x}}(P)=1 .
\end{aligned}
$$

6d vielbeins. To study the global anomalies in $6 \mathrm{~d}$ we evaluate $\theta_{\mu}^{a}(B)$ for the metric in (4.8) using the following vielbeins

$$
\begin{aligned}
& e_{r}^{\hat{r}}(B)=1, \quad e_{y}^{\hat{y}}(B)=1, \quad e_{x}^{\hat{x}}(B)=1, \quad e_{z}^{\hat{z}}(B)=1 \\
& e_{c}^{\hat{c}}(B)=1, \quad e_{b}^{\hat{b}}(B)=1, \quad e_{e}^{\hat{e}}(B)=1, \quad e_{t}^{\hat{t}}(B)=f(r), \\
& e_{x}^{\hat{t}}(B)=f(r)\left(a_{1}(a)+2 u\right), \quad e_{z}^{\hat{t}}(B)=f(r) a_{2}(b), \quad e_{b}^{\hat{t}}=f(r) a_{3}(y) .
\end{aligned}
$$

For the product metric at the boundary given in (4.10) the vielbeins are

$$
\begin{aligned}
& e_{r}^{\hat{r}}(P)=1, \quad e_{y}^{\hat{y}}(P)=1, \quad e_{x}^{\hat{x}}(P)=1, \quad e_{z}^{\hat{z}}(P)=1, \\
& e_{c}^{\hat{c}}(P)=1, \quad e_{b}^{\hat{b}}(P)=1, \quad e_{e}^{\hat{e}}(P)=1, \quad e_{t}^{\hat{t}}(P)=f(1), \\
& e_{x}^{\hat{t}}(P)=f(1)\left(a_{1}(a)+2 u\right), \quad e_{z}^{\hat{t}}(P)=f(1) a_{2}(b), \quad e_{b}^{\hat{t}}(P)=f(1) a_{3}(y) .
\end{aligned}
$$

Curvature integrals. To evaluate the $\eta$ invariant in $d=6$ we require the following curvature integrals of the metric in (4.8) as well as boundary terms associated with the 
metric in (4.10)

$$
\begin{aligned}
I_{1}= & \int_{B} \operatorname{Tr} R \wedge R \wedge R \wedge R \\
= & \int_{B} 2 a_{2}^{\prime}(b) a_{3}^{\prime}(y) f^{\prime}(r)\left\{f(r)^{7}\left[5+5 a_{2}^{\prime}(b)^{4}-a_{3}^{\prime}(y)^{2}+5 a_{3}^{\prime}(y)^{4}-a_{2}^{\prime}(b)^{2}\left(1+a_{3}^{\prime}(y)^{2}\right)\right]\right. \\
& \left.+24 f^{\prime}(r)^{2} f^{\prime \prime}(r)\right\}, \\
= & \int d t d a d b d x d y d z \times \\
& 4\left\{a _ { 2 } ^ { \prime } ( b ) a _ { 3 } ^ { \prime } ( y ) \left[\frac { f ( 1 ) ^ { 8 } } { 8 } \left(5+5 a_{2}^{\prime}(b)^{4}-a_{3}^{\prime}(y)^{2}+5 a_{3}^{\prime}(y)^{4}\right.\right.\right. \\
& \left.\left.\left.-a_{2}^{\prime}(b)^{2}\left(1+a_{3}^{\prime}(y)^{2}\right)\right)+6 f^{\prime}(1)^{4}-6\right]\right\}, \\
I_{\theta 1}= & \int_{\Sigma} \operatorname{Tr} \theta \wedge R \wedge R \wedge R,=24 \int d t d a d b d x d y d z f^{\prime}(1)^{4} a_{2}^{\prime}(b) a_{3}^{\prime}(y), \\
I_{2}= & \int_{\operatorname{Tr}} R \wedge R \wedge \operatorname{Tr} R \wedge R, \\
= & \int_{B} 4 a_{2}^{\prime}(b) a_{3}^{\prime}(y) f^{\prime}(r)\left\{f(r)^{7}\left[5+5 a_{2}^{\prime}(b)^{4}+11 a_{3}^{\prime}(y)^{2}+5 a_{3}^{\prime}(y)^{4}+11 a_{2}^{\prime}(b)^{2}\left(1+a_{3}^{\prime}(y)^{2}\right)\right]\right. \\
& \left.+16 f(r)^{3}\left(1+a_{2}^{\prime}(b)^{2}+a_{3}^{\prime}(y)^{2}\right) f^{\prime}(r)^{2}+8\left[f(r)^{4}\left(1+a_{2}^{\prime}(b)^{2}+a_{3}^{\prime}(y)^{2}\right)+3 f^{\prime}(r)^{2}\right] f^{\prime \prime}(r)\right\}, \\
= & \int_{d t d a d b d x d y d z 8 a_{2}^{\prime}(b) a_{3}^{\prime}(y) \times} \\
& \left\{\frac{f(1)^{8}}{8}\left[5+5 a_{2}^{\prime}(b)^{4}+11 a_{3}^{\prime}(y)^{2}+5 a_{3}^{\prime}(y)^{4}+11 a_{2}^{\prime}(b)^{2}\left(1+a_{3}^{\prime}(y)^{2}\right)\right]\right. \\
& \left.+4 f(1)^{4} f^{\prime}(1)^{2}\left(1+a_{2}^{\prime}(b)^{2}+a_{3}^{\prime}(y)^{2}\right)+6 f^{\prime}(1)^{4}-6\right\}, \\
I_{\theta 2}= & \int_{\Sigma} \operatorname{Tr} \theta \wedge R \operatorname{Tr} R \wedge R,=\int d t d a d b d x d y d z \times \\
& \left\{-\frac{1}{2} a_{2}^{\prime}(b) a_{3}^{\prime}(y)\left[-32 f(1)^{4} f^{\prime}(1)^{2}\left(1+a_{2}^{\prime}(b)^{2}+a_{3}^{\prime}(y)^{2}\right)-96 f^{\prime}(1)^{4}\right]\right\}, \\
& \\
&
\end{aligned}
$$

where the orientation is decided by $\epsilon^{\text {taxybur }}=1$.

Chern Simons terms. We obtain identities that relate the terms in the anomaly polynomial $p_{1}^{2}$ and $p_{2}^{2}$ to exterior derivatives of Chern-Simons terms. We then evaluate these Chern-Simons terms for the metric (4.10).

From the definition of the curvature form we have the identity

$$
\begin{aligned}
\operatorname{Tr}(R \wedge R \wedge R \wedge R)= & \operatorname{Tr}(d \omega \wedge d \omega \wedge d \omega \wedge d \omega+4 d \omega \wedge d \omega \wedge d \omega \wedge \omega \wedge \omega \\
& +4 d \omega \wedge d \omega \wedge \omega \wedge \omega \wedge \omega \wedge \omega+2 d \omega \wedge \omega \wedge \wedge \omega d \omega \wedge \omega \wedge \omega \wedge \omega \\
& +4 d \omega \wedge \omega \wedge \omega \wedge \omega \wedge \omega \wedge \omega \wedge \omega)
\end{aligned}
$$


We observe that each term in the r.h.s. of the above equation can be written as an exact form using the following identities

$$
\begin{aligned}
d[\operatorname{Tr}(\omega \wedge d \omega \wedge d \omega \wedge d \omega)] & =\operatorname{Tr} d \omega \wedge d \omega \wedge d \omega \wedge d \omega), \\
d[\operatorname{Tr}(d \omega \wedge \omega \wedge \omega \wedge \omega \wedge \omega \wedge \omega)] & =2 \operatorname{Tr}(d \omega \wedge d \omega \wedge \omega \wedge \omega \wedge \omega \wedge \omega) \\
& +\operatorname{Tr}(d \omega \wedge \omega \wedge \omega \wedge d \omega \wedge \omega \wedge \omega), \\
\frac{2}{5} d\left[\operatorname{Tr}(d \omega \wedge d \omega \wedge \omega \wedge \omega \wedge \omega)+\frac{1}{2} \operatorname{Tr}(d \omega \wedge \omega \wedge d \omega \wedge \omega \wedge \omega)\right] & =\operatorname{Tr}(d \omega \wedge d \omega \wedge d \omega \wedge \omega \wedge \omega), \\
\frac{1}{7} d[\operatorname{Tr}(\omega \wedge \omega \wedge \omega \wedge \omega \wedge \omega \wedge \omega \wedge \omega)] & =\operatorname{Tr}(d \omega \wedge \omega \wedge \omega \wedge \omega \wedge \omega \wedge \omega \wedge \omega) .
\end{aligned}
$$

Combining all these identities we obtain

$$
\begin{aligned}
\operatorname{Tr}(R \wedge R \wedge R \wedge R)= & d\left[\operatorname{Tr}(\omega \wedge d \omega \wedge d \omega \wedge d \omega)+\frac{8}{5} \operatorname{Tr}(d \omega \wedge d \omega \wedge \omega \wedge \omega \wedge \omega)\right. \\
& +\frac{4}{5} \operatorname{Tr}(d \omega \wedge \omega \wedge d \omega \wedge \omega \wedge \omega)+\frac{4}{7} \operatorname{Tr}(\omega \wedge \omega \wedge \omega \wedge \omega \wedge \omega \wedge \omega \wedge \omega) \\
& +2 \operatorname{Tr}(d \omega \wedge \omega \wedge \omega \wedge \omega \wedge \omega \wedge \omega)]
\end{aligned}
$$

Similarly,

$$
\operatorname{Tr}(R \wedge R) \operatorname{Tr}(R \wedge R)=d\left[\operatorname{Tr}\left(\omega \wedge d \omega+\frac{2}{3} \omega \wedge \omega \wedge \omega\right) \wedge \operatorname{Tr}(R \wedge R)\right]
$$

Finally we can write down the anomaly polynomials as exterior derivatives of ChernSimons terms.

$$
\begin{gathered}
p_{1}(\mathcal{R})^{2}=\frac{1}{4(2 \pi)^{4}} \frac{\operatorname{Tr}(R \wedge R)^{2}}{4}, \\
=\frac{1}{(2 \pi)^{4}} d\left[\operatorname{Tr}\left(\omega \wedge d \omega+\frac{2}{3} \omega \wedge \omega \wedge \omega\right) \wedge \operatorname{Tr}(R \wedge R)\right] . \\
p_{2}(\mathcal{R})=\frac{-1}{(2 \pi)^{4}}\left(\frac{1}{4} \operatorname{Tr}(R \wedge R \wedge R \wedge R)-\frac{1}{8} \operatorname{Tr}(R \wedge R) \operatorname{Tr}(R \wedge R)\right), \\
=\frac{-1}{4(2 \pi)^{4}} d\left[\operatorname{Tr}(\omega \wedge d \omega \wedge d \omega \wedge d \omega)+\frac{8}{5} \operatorname{Tr}(d \omega \wedge d \omega \wedge \omega \wedge \omega \wedge \omega)\right. \\
+\frac{4}{5} \operatorname{Tr}(d \omega \wedge \omega \wedge d \omega \wedge \omega \wedge \omega)++\frac{4}{7} \operatorname{Tr}(\omega \wedge \omega \wedge \omega \wedge \omega \wedge \omega \wedge \omega \wedge \omega) \\
\left.+2 \operatorname{Tr}(d \omega \wedge \omega \wedge \omega \wedge \omega \wedge \omega \wedge \omega)-\frac{1}{2} \operatorname{Tr}\left(\omega \wedge d \omega+\frac{2}{3} \omega \wedge \omega \wedge \omega\right) \wedge \operatorname{Tr}(R \wedge R)\right] .
\end{gathered}
$$


Chern Simons integrals. We will require integrals of the various Chern Simons forms over the boundary of the metric given in (4.8). These are given by

$$
\begin{aligned}
\int_{\Sigma} \operatorname{Tr} \omega \wedge d \omega \wedge d \omega \wedge d \omega & =-\frac{3(2 \pi)^{4}}{2} \int d b d y f(1)^{8} a_{2}^{\prime}(b) a_{3}^{\prime}(y)\left[\left(a_{2}^{\prime}(b)^{4}+a_{3}^{\prime}(y)^{4}\right)+1\right] \\
\int_{\Sigma} \operatorname{Tr} d \omega \wedge d \omega \wedge \omega \wedge \omega \wedge \omega & =-\frac{(2 \pi)^{4}}{2} \int d b d y f(1)^{8} a_{2}^{\prime}(b) a_{3}^{\prime}(y)\left[\left(a_{2}^{\prime}(b)^{4}+a_{3}^{\prime}(y)^{4}\right)+1\right], \\
\int_{\Sigma} \operatorname{Tr} d \omega \wedge \omega \wedge d \omega \wedge \omega \wedge \omega= & -\frac{(2 \pi)^{4}}{4} \int d b d y f(1)^{8} a_{2}^{\prime}(b) a_{3}^{\prime}(y)\left[\left(a_{2}^{\prime}(b)^{4}+a_{3}^{\prime}(y)^{4}\right)+1\right], \\
\int_{\Sigma} \operatorname{Tr} d \omega \wedge \omega \wedge \omega \wedge \omega \wedge \omega \wedge \omega= & \frac{(2 \pi)^{4}}{4} \int d b d y f(1)^{8} a_{2}^{\prime}(b) a_{3}^{\prime}(y)\left[a_{2}^{\prime}(b)^{2}\left(a_{3}^{\prime}(y)^{2}+1\right)+a_{3}^{\prime}(y)^{2}\right], \\
\int_{\Sigma} \operatorname{Tr} \omega \wedge \omega \wedge \omega \wedge \omega \wedge \omega \wedge \omega \wedge \omega= & 0, \\
\int_{\Sigma} \operatorname{Tr}\left(\omega \wedge d \omega+\frac{2}{3} \omega \wedge \omega \wedge \omega\right) \operatorname{Tr}(R \wedge R)= & (2 \pi)^{4} f(1)^{4} \int d b d y a_{2}^{\prime}(b) a_{3}^{\prime}(y) \times \\
& \left\{-16 f^{\prime}(1)^{2}\left(a_{2}^{\prime}(b)^{2}+a_{3}^{\prime}(y)^{2}+1\right)\right. \\
& -f(1)^{4}\left[11 a_{2}^{\prime}(b)^{2}\left(a_{3}^{\prime}(y)^{2}+1\right)+5 a_{2}^{\prime}(b)^{4}\right. \\
& \left.\left.+5 a_{3}^{\prime}(y)^{4}+11 a_{3}^{\prime}(y)^{2}+5\right]\right\} .
\end{aligned}
$$

In the last integral it is important to note that the curvature form $\operatorname{Tr}(R \wedge R)$ is evaluated from the metric (4.8) but at the boundary.

\section{B Correlators of self dual tensors in $d=6$}

In this appendix we present the details involved in evaluating the Kubo formula (5.4) to obtain the transport coefficient $\lambda_{3(S)}^{(6)}$ We first write down the components of the flat space stress tensors we will use explicitly. This will facilitate the discussion of the Wick contractions.

$$
\begin{aligned}
T_{f l}^{\tau a}(p+k)= & \frac{-1}{\beta} \sum_{\omega_{m}} \int \frac{d^{5} p_{1}}{(2 \pi)^{5}}\left[\frac{1}{2}\left(F^{\tau b x}+i F^{a y z}\right)\left(-p_{1}+p+k\right)\left(F^{a b x}-i F^{\tau y z}\right)\left(p_{1}\right)\right. \\
& +\frac{1}{2}\left(F^{\tau b y}-i F^{a x z}\right)\left(-p_{1}+p+k\right)\left(F^{a b y}+i F^{\tau x z}\right)\left(p_{1}\right) \\
& \left.+\frac{1}{2}\left(F^{\tau b z}-i F^{a y x}\right)\left(-p_{1}+p+k\right)\left(F^{a b z}+i F^{\tau y x}\right)\left(p_{1}\right)\right], \\
T_{f l}^{\tau x}(-k)= & \frac{-1}{\beta} \sum_{\omega_{m}} \int \frac{d^{5} p_{2}}{(2 \pi)^{5}}\left[\left(F^{\tau a b}\left(-p_{2}-k\right) F^{x a b}\left(p_{2}\right)+F^{\tau a y}\left(-p_{2}-k\right) F^{x a y}\left(p_{2}\right)\right.\right. \\
& +F^{\tau a z}\left(-p_{2}-k\right) F^{x a z}\left(p_{2}\right)+F^{\tau b y}\left(-p_{2}-k\right) F^{x b y}\left(p_{2}\right) \\
& \left.+F^{\tau b z}\left(-p_{2}-k\right) F^{x b z}\left(p_{2}\right)+F^{\tau y z}\left(-p_{2}-k\right) F^{x y z}\left(p_{2}\right)\right] \\
T_{f l}^{\tau z}(-p)= & \frac{-1}{\beta} \sum_{\omega_{m}} \int \frac{d^{5} p_{3}}{(2 \pi)^{5}}\left[F^{\tau a b}\left(-p_{3}-p\right) F^{z a b}\left(p_{3}\right)+F^{\tau a y}\left(-p_{3}-p\right) F^{z a y}\left(p_{3}\right)\right. \\
& +F^{\tau a x}\left(-p_{3}-p\right) F^{z a x}\left(p_{3}\right)+F^{\tau b y}\left(-p_{3}-p\right) F^{z b y}\left(p_{3}\right) \\
& \left.+F^{\tau b x}\left(-p_{3}-p\right) F^{z b x}\left(p_{3}\right)+F^{\tau y x}\left(-p_{3}-p\right) F^{z y x}\left(p_{3}\right)\right] .
\end{aligned}
$$


We have imposed the self dual projection on $T^{\tau a}$, also note that after summing over the indices involved in $F$ we obtain an overall factor of $1 / 2$. Again in writing down $T^{\tau x}$ and $T^{\tau z}$, the indices in $F$ has been summed over.

The kinematic configurations is same as the one used in [16] for $d=6$. The two external momenta are labelled as $p, k$. The momentum $p$ has non-zero component only in the $b$ direction, while $k$ has non-zero momentum only in the $y$ direction. The one loop internal momenta is labelled by $p_{3}$. To perform the integration over $p_{3}$, we will parametrize its components in terms of angular variables

$$
\begin{array}{r}
p_{3}^{b}=\left|p_{3}\right| \cos \phi_{1}, \quad p_{3}^{y}=\left|p_{3}\right| \sin \phi_{1} \cos \phi_{2}, \quad p_{3}^{x}=\left|p_{3}\right| \sin \phi_{1} \sin \phi_{2} \cos \phi_{3}, \\
p_{3}^{z}=\sin \phi_{1} \sin \phi_{2} \sin \phi_{3} \cos \phi_{4}, \quad p_{3}^{a}=\sin \phi_{1} \sin \phi_{2} \sin \phi_{3} \sin \phi_{4} .
\end{array}
$$

With this kinematic configuration, we write down the expression for the energies which will occur in the expression for the propagators.

$$
\begin{aligned}
E_{p_{3}+p} & =\left(\left|p_{3}\right|^{2}+|p|^{2}+2|p|\left|p_{3}\right| \cos \phi_{1}\right)^{\frac{1}{2}}, \\
E_{p 3+k} & =\left(\left|p_{3}\right|^{2}+|k|^{2}+2|k|\left|p_{3}\right| \sin \phi_{1} \cos \phi_{2}\right)^{\frac{1}{2}}, \\
E_{p 3-k} & =\left(\left|p_{3}\right|^{2}+|k|^{2}-2|k|\left|p_{3}\right| \sin \phi_{1} \cos \phi_{2}\right)^{\frac{1}{2}}, \\
E_{p_{3}+p+k} & =\left(\left|p_{3}\right|^{2}+|k|^{2}+|p|^{2}+2|k|\left|p_{3}\right| \sin \phi_{1} \cos \phi_{2}+2|p|\left|p_{3}\right| \cos \phi_{1}\right)^{\frac{1}{2}} .
\end{aligned}
$$

In order to keep track of the Wick contractions, we adopt the following convention. We will denote the $i^{\text {th }}$ term in the expression for the stress tensor as, $T_{i}^{\mu \nu}$. For example,

$$
T_{2}^{\tau x}=\frac{-1}{\beta} \sum_{\omega_{n}} \int \frac{d^{5} p_{2}}{(2 \pi)^{5}} F^{\tau a y}\left(-p_{2}-k\right) F^{x a y}\left(p_{2}\right) .
$$

From (5.12) and (5.4), we see that the general Wick contraction structure looks like,

$$
I=T_{i}^{\tau a} T_{j}^{\tau x} T_{k}^{\tau z}
$$

where $i, j, k$ denote the respective terms in the expression for the stress tensor.

Contractions with odd number of $\tau$ 's vanish. There is one important simplification when we consider the Wick contractions before a detailed evaluation. Note that the Matsubara sums run from negative infinity to positive infinity. Applying (5.10) to such a generic contraction, it is easy to see that the Matsubara sum gives non zero contribution only when the terms involved in the Wick contraction have even number of $\tau$ indices. Let 
us illustrate this claim with the help of an example,

$$
\begin{aligned}
\left\langle T_{1}^{\tau a} T_{3}^{\tau x} T_{1}^{\tau z}\right\rangle= & \frac{-1}{\beta} \sum_{\omega_{n}^{1}} \int \frac{d^{5} p_{1}}{(2 \pi)^{5}} \frac{-1}{\beta} \sum_{\omega_{n}^{2}} \int \frac{d^{5} p_{2}}{(2 \pi)^{5}} \frac{-1}{\beta} \sum_{\omega_{n}^{3}} \int \frac{d^{5} p_{3}}{(2 \pi)^{5}}\left\langle\frac{1}{2} F^{\tau b x}\left(-p_{1}+p+k\right) F^{\tau y z}\left(p_{1}\right)\right. \\
& \left.F^{\tau a z}\left(-p_{2}-k\right) F^{x a z}\left(p_{2}\right) F^{\tau a b}\left(-p_{3}-p\right) F^{z a b}\left(p_{3}\right)\right\rangle, \\
= & \frac{-1}{\beta} \sum_{\omega_{n}^{3}} \int \frac{d^{5} p_{3}}{(2 \pi)^{5}}\left\langle F^{a b x}\left(p_{3}-k\right) F^{a z x}\left(-p_{3}+k\right)\right\rangle\left\langle F^{\tau a z}\left(p_{3}\right) F^{a z b}\left(-p_{3}\right)\right\rangle \\
& \left\langle F^{\tau b x}\left(p_{3}+p\right) F^{\tau b a}\left(-p_{3}-p\right)\right\rangle, \\
= & \frac{-1}{\beta} \sum_{\omega_{n}^{3}} \int \frac{d^{5} p_{3}}{(2 \pi)^{5}} \frac{-i}{2} \frac{\left(p_{3}^{b}\right)^{2} p_{3}^{z} p_{3}^{x} p_{3}^{a}\left(i \omega_{n}^{3}\right)}{\left(\left(i \omega_{n}^{3}\right)^{2}-E_{p_{3}-k}^{2}\right)\left(\left(i \omega_{n}^{3}\right)^{2}-E_{p_{3}}^{2}\right)\left(\left(i \omega_{n}^{3}\right)^{2}-E_{p_{3}+p}^{2}\right)} .
\end{aligned}
$$

The sum over the Matsubara frequencies in the above expression is from negative infinity to positive infinity. Note that the sum runs over an odd function of $\omega^{3}$. Therefore the result vanishes.

Therefore using the observation in the previous paragraph we can conclude that the terms in the stress tensor $T^{\tau a}$ that result in non-zero wick contractions are,

$$
\begin{aligned}
T^{\tau a}(p+k)= & \frac{-1}{\beta} \sum_{\omega_{m}} \int \frac{d^{5} p_{1}}{(2 \pi)^{5}}\left(-\frac{i}{2} F^{\tau b x}\left(-p_{1}+p+k\right) F^{\tau y z}\left(p_{1}\right)+\frac{i}{2} F^{a y z}\left(-p_{1}+p+k\right) F^{a b x}\left(p_{1}\right)\right. \\
& +\frac{i}{2} F^{\tau b y}\left(-p_{1}+p+k\right) F^{\tau x z}\left(p_{1}\right)-\frac{i}{2} F^{a x z}\left(-p_{1}+p+k\right) F^{a b y}\left(p_{1}\right) \\
& \left.+\frac{i}{2} F^{\tau b z}\left(-p_{1}+p+k\right) F^{\tau y x}\left(p_{1}\right)-\frac{i}{2} F^{a y x}\left(-p_{1}+p+k\right) F^{a b z}\left(p_{1}\right)\right) .
\end{aligned}
$$

This is because the expansion of the stress tensor $T^{\tau x}$ and $T^{\tau z}$ contains a single $\tau$ in each of its terms.

The resulting Wick contractions even after this simplification, are numerous. We have developed a Mathematica code to perform the Wick contractions. These Wick contractions can be broadly classified into two types depending on the denominators. We illustrate this fact with the following examples.

$$
\begin{aligned}
\left\langle T_{1}^{\tau a} T_{1}^{\tau x} T_{2}^{\tau z}\right\rangle= & \frac{-1}{\beta} \sum_{\omega_{n}^{1}} \int \frac{d^{5} p_{1}}{(2 \pi)^{5}} \frac{1}{\beta} \sum_{\omega_{n}^{2}} \int \frac{d^{5} p_{2}}{(2 \pi)^{5}} \frac{1}{\beta} \sum_{\omega_{n}^{3}} \int \frac{d^{5} p_{3}}{(2 \pi)^{5}}\left\langle-\frac{i}{2} F^{\tau b x}\left(-p_{1}+p+k\right) F^{\tau y z}\left(p_{1}\right)\right. \\
& \left.\times F^{\tau a b}\left(-p_{2}-k\right) F^{x a b}\left(p_{2}\right) F^{\tau a y}\left(-p_{3}-p\right) F^{z a y}\left(p_{3}\right)\right\rangle, \\
= & \frac{1}{\beta} \sum_{\omega_{n}^{3}} \int \frac{d^{5} p_{3}}{(2 \pi)^{5}} \frac{i}{2} \frac{\left\{-p_{3}\right\}^{a}\left\{-p_{3}\right\}^{\tau}\left\{p+p_{3}\right\}^{b}\left\{p+p_{3}\right\}^{y}\left\{k+p+p_{3}\right\}^{a}\left\{k+p+p_{3}\right\}^{\tau}}{\left(i \omega^{2}-E_{\left(p_{3}\right)^{2}}\right)\left(i \omega^{2}-E_{\left(p+p_{3}\right)^{2}}\right)\left(i \omega^{2}-E_{\left(k+p+p_{3}\right)^{2}}\right)} . \\
\left\langle T_{5}^{\tau a} T_{1}^{\tau x} T_{5}^{\tau z}\right\rangle= & \frac{-1}{\beta} \sum_{\omega_{n}^{1}} \int \frac{d^{5} p_{1}}{(2 \pi)^{5}} \frac{1}{\beta} \sum_{\omega_{n}^{2}} \int \frac{d^{5} p_{2}}{(2 \pi)^{5}} \frac{1}{\beta} \sum_{\omega_{n}^{3}} \int \frac{d^{5} p_{3}}{(2 \pi)^{5}}\left\langle\frac{i}{2} F^{\tau b z}\left(-p_{1}+p+k\right) F^{\tau y x}\left(p_{1}\right)\right. \\
& \left.\times F^{\tau a b}\left(-p_{2}-k\right) F^{x a b}\left(p_{2}\right) F^{\tau b x}\left(-p_{3}-p\right) F^{z b x}\left(p_{3}\right)\right\rangle, \\
= & \frac{1}{\beta} \sum_{\omega_{n}^{3}} \int \frac{d^{5} p_{3}}{(2 \pi)^{5}} \frac{i}{2} \frac{\left\{-p_{3}\right\}^{a}\left\{-p_{3}\right\}^{z}\left\{k-p_{3}\right\}^{a}\left\{p+p_{3}\right\}^{b}\left\{k-p_{3}\right\}^{z}\left\{p+p_{3}\right\}^{y}}{\left(i \omega^{2}-E_{\left(-p_{3}\right)^{2}}\right)\left(i \omega^{2}-E_{\left(k-p_{3}\right)^{2}}\right)\left(i \omega^{2}-E_{\left(p+p_{3}\right)^{2}}\right)}
\end{aligned}
$$


Result of the Wick contractions. All the Wick contractions fall into two classes depending on the denominator. They are given by terms of the kind

$$
\begin{aligned}
& W_{A}=\frac{1}{\beta} \sum_{\omega_{n}^{3}} \int \frac{d^{5} p_{3}}{(2 \pi)^{5}} c_{A}, \\
& c_{A}=\left\{\left[-i\left(k^{y}\right)^{2}\left(\left(p_{3}^{a}\right)^{2} p_{3}^{b} p_{3}^{y}+p_{3}^{b}\left(p_{3}^{\tau}\right)^{2} p_{3}^{y}+p_{3}^{b}\left(p_{3}^{x}\right)^{2} p_{3}^{y}+p_{3}^{b} p_{3}^{y}\left(p_{3}^{z}\right)^{2}\right.\right.\right. \\
& \left.+\left(p_{3}^{b}\right)^{3} p_{3}^{y}+p_{3}^{b}\left(p_{3}^{y}\right)^{3}\right)-i p_{3}^{b} k^{y}\left(\left(p_{3}^{a}\right)^{2}+\left(p_{3}^{b}\right)^{2}-\left(p_{3}^{\tau}\right)^{2}+\left(p_{3}^{x}\right)^{2}+\left(p_{3}^{y}\right)^{2}+\left(p_{3}^{z}\right)^{2}\right) \\
& \left.\left(\left(p_{3}^{a}\right)^{2}+\left(p_{3}^{b}\right)^{2}+\left(p_{3}^{\tau}\right)^{2}+\left(p_{3}^{x}\right)^{2}+\left(p_{3}^{y}\right)^{2}+\left(p_{3}^{z}\right)^{2}\right)\right]+\left[-i k^{y}\left(( p _ { 3 } ^ { a } ) ^ { 2 } \left(4\left(p_{3}^{b}\right)^{2}-3\left(p_{3}^{\tau}\right)^{2}\right.\right.\right. \\
& \left.+2\left(\left(p_{3}^{x}\right)^{2}+\left(p_{3}^{z}\right)^{2}\right)\right)+\left(p_{3}^{a}\right)^{4}+\left(p_{3}^{b}\right)^{2}\left(-\left(p_{3}^{\tau}\right)^{2}+4\left(p_{3}^{x}\right)^{2}+2\left(p_{3}^{y}\right)^{2}+4\left(p_{3}^{z}\right)^{2}\right)+3\left(p_{3}^{b}\right)^{4} \\
& \left.-\left(\left(p_{3}^{x}\right)^{2}+\left(p_{3}^{y}\right)^{2}+\left(p_{3}^{z}\right)^{2}\right)\left(3\left(p_{3}^{\tau}\right)^{2}-\left(p_{3}^{x}\right)^{2}+\left(p_{3}^{y}\right)^{2}-\left(p_{3}^{z}\right)^{2}\right)\right)+i p_{3}^{y}\left(\left(p_{3}^{a}\right)^{2}+\left(p_{3}^{b}\right)^{2}\right. \\
& \left.+\left(p_{3}^{x}\right)^{2}+\left(p_{3}^{y}\right)^{2}+\left(p_{3}^{z}\right)^{2}\right)\left(\left(p_{3}^{a}\right)^{2}+\left(p_{3}^{b}\right)^{2}+\left(p_{3}^{\tau}\right)^{2}+\left(p_{3}^{x}\right)^{2}+\left(p_{3}^{y}\right)^{2}+\left(p_{3}^{z}\right)^{2}\right) \\
& \left.-i\left(k^{y}\right)^{2}\left(\left(p_{3}^{b}\right)^{2} p_{3}^{y}-\left(p_{3}^{\tau}\right)^{2} p_{3}^{y}+p_{3}^{y}\left(p_{3}^{z}\right)^{2}\right)\right] p^{b}+\left[k ^ { y } \left(-3 i\left(p_{3}^{a}\right)^{2} p_{3}^{b}+i p_{3}^{b}\left(p_{3}^{\tau}\right)^{2}\right.\right. \\
& \left.-2 i p_{3}^{b}\left(p_{3}^{x}\right)^{2}-3 i p_{3}^{b}\left(p_{3}^{z}\right)^{2}-3 i\left(p_{3}^{b}\right)^{3}\right)+\left(3 i\left(p_{3}^{a}\right)^{2} p_{3}^{b} p_{3}^{y}+i p_{3}^{b}\left(p_{3}^{\tau}\right)^{2} p_{3}^{y}\right. \\
& \left.\left.+3 i p_{3}^{b}\left(p_{3}^{x}\right)^{2} p_{3}^{y}+3 i p_{3}^{b} p_{3}^{y}\left(p_{3}^{z}\right)^{2}+3 i\left(p_{3}^{b}\right)^{3} p_{3}^{y}+3 i p_{3}^{b}\left(p_{3}^{y}\right)^{3}\right)\right]\left(p^{b}\right)^{2} \\
& +\left[k^{y}\left(-i\left(p_{3}^{a}\right)^{2}-i\left(p_{3}^{b}\right)^{2}-i\left(p_{3}^{z}\right)^{2}\right)+\left(i\left(p_{3}^{a}\right)^{2} p_{3}^{y}+3 i\left(p_{3}^{b}\right)^{2} p_{3}^{y}+i\left(p_{3}^{x}\right)^{2} p_{3}^{y}\right.\right. \\
& \left.\left.\left.+i p_{3}^{y}\left(p_{3}^{z}\right)^{2}+i\left(p_{3}^{y}\right)^{3}\right)\right]\left(p^{b}\right)^{3}+i\left(p^{b}\right)^{4} p_{3}^{b} p_{3}^{y}\right\} \\
& \times \frac{-1}{2\left(i \omega^{2}-E_{-p_{3}}^{2}\right)\left(i \omega^{2}-E_{p+p_{3}}^{2}\right)\left(i \omega^{2}-E_{k+p+p_{3}}^{2}\right)} \text {. } \\
& W_{B}=\frac{1}{\beta} \sum_{\omega_{n}^{3}} \int \frac{d^{5} p_{3}}{(2 \pi)^{5}} c_{B}, \\
& c_{B}=\left\{\left[i ( k ^ { y } ) ^ { 2 } \left(\left(p_{3}^{a}\right)^{2} p_{3}^{b} p_{3}^{y}+p_{3}^{b}\left(p_{3}^{\tau}\right)^{2} p_{3}^{y}+p_{3}^{b}\left(p_{3}^{x}\right)^{2} p_{3}^{y}+p_{3}^{b} p_{3}^{y}\left(p_{3}^{z}\right)^{2}+\left(p_{3}^{b}\right)^{3} p_{3}^{y}\right.\right.\right. \\
& \left.+p_{3}^{b}\left(p_{3}^{y}\right)^{3}\right)-i p_{3}^{b} k^{y}\left(\left(p_{3}^{a}\right)^{2}+\left(p_{3}^{b}\right)^{2}-\left(p_{3}^{\tau}\right)^{2}+\left(p_{3}^{x}\right)^{2}+\left(p_{3}^{y}\right)^{2}+\left(p_{3}^{z}\right)^{2}\right)\left(\left(p_{3}^{a}\right)^{2}+\left(p_{3}^{b}\right)^{2}\right. \\
& \left.\left.+\left(p_{3}^{\tau}\right)^{2}+\left(p_{3}^{x}\right)^{2}+\left(p_{3}^{y}\right)^{2}+\left(p_{3}^{z}\right)^{2}\right)\right]+\left[-i k^{y}\left(3\left(p_{3}^{a}\right)^{2}\left(p_{3}^{b}\right)^{2}-3\left(p_{3}^{a}\right)^{2}\left(p_{3}^{\tau}\right)^{2}+2\left(p_{3}^{a}\right)^{2}\left(p_{3}^{x}\right)^{2}\right.\right. \\
& +3\left(p_{3}^{a}\right)^{2}\left(p_{3}^{y}\right)^{2}+2\left(p_{3}^{a}\right)^{2}\left(p_{3}^{z}\right)^{2}+\left(p_{3}^{a}\right)^{4}-2\left(p_{3}^{b}\right)^{2}\left(p_{3}^{\tau}\right)^{2}+3\left(p_{3}^{b}\right)^{2}\left(p_{3}^{x}\right)^{2}+4\left(p_{3}^{b}\right)^{2}\left(p_{3}^{y}\right)^{2} \\
& +3\left(p_{3}^{b}\right)^{2}\left(p_{3}^{z}\right)^{2}+2\left(p_{3}^{b}\right)^{4}-3\left(p_{3}^{\tau}\right)^{2}\left(p_{3}^{x}\right)^{2}-3\left(p_{3}^{\tau}\right)^{2}\left(p_{3}^{z}\right)^{2}+3\left(p_{3}^{x}\right)^{2}\left(p_{3}^{y}\right)^{2} \\
& \left.+2\left(p_{3}^{x}\right)^{2}\left(p_{3}^{z}\right)^{2}+\left(p_{3}^{x}\right)^{4}+3\left(p_{3}^{y}\right)^{2}\left(p_{3}^{z}\right)^{2}+2\left(p_{3}^{y}\right)^{4}+\left(p_{3}^{z}\right)^{4}\right)+i\left(k^{y}\right)^{2}\left(\left(p_{3}^{a}\right)^{2} p_{3}^{y}\right. \\
& \left.+2\left(p_{3}^{b}\right)^{2} p_{3}^{y}+\left(p_{3}^{x}\right)^{2} p_{3}^{y}+2 p_{3}^{y}\left(p_{3}^{z}\right)^{2}+\left(p_{3}^{y}\right)^{3}\right)+i p_{3}^{y}\left(\left(p_{3}^{a}\right)^{2}\right.
\end{aligned}
$$




$$
\begin{aligned}
& \left.\left.+\left(p_{3}^{b}\right)^{2}+\left(p_{3}^{x}\right)^{2}+\left(p_{3}^{y}\right)^{2}+\left(p_{3}^{z}\right)^{2}\right)\left(\left(p_{3}^{a}\right)^{2}+\left(p_{3}^{b}\right)^{2}+\left(p_{3}^{\tau}\right)^{2}+\left(p_{3}^{x}\right)^{2}+\left(p_{3}^{y}\right)^{2}+\left(p_{3}^{z}\right)^{2}\right)\right] p^{b} \\
& +\left[-i k^{y}\left(\left(p_{3}^{a}\right)^{2} p_{3}^{b}-p_{3}^{b}\left(p_{3}^{\tau}\right)^{2}+2 p_{3}^{b}\left(p_{3}^{x}\right)^{2}+2 p_{3}^{b}\left(p_{3}^{y}\right)^{2}+p_{3}^{b}\left(p_{3}^{z}\right)^{2}+\left(p_{3}^{b}\right)^{3}\right.\right. \\
& \left.\left.+i p_{3}^{b} p_{3}^{y}\left(\left(p_{3}^{a}\right)^{2}+\left(p_{3}^{b}\right)^{2}+\left(p_{3}^{\tau}\right)^{2}+\left(p_{3}^{x}\right)^{2}+\left(p_{3}^{y}\right)^{2}+\left(p_{3}^{z}\right)^{2}\right)+i p_{3}^{b}\left(k^{y}\right)^{2} p_{3}^{y}\right]\left(p^{b}\right)^{2}\right\} \\
& \times \frac{-1}{2\left(i \omega^{2}-E_{k-p_{3}}^{2}\right)\left(i \omega^{2}-E_{-p_{3}}^{2}\right)\left(i \omega^{2}-E_{p+p_{3}}^{2}\right)} .
\end{aligned}
$$

From the analysis it is evident that Wick contractions in (B.8) belongs to class (B.10) while the contractions in (B.9) belongs to Class (B.11)

Contractions organised as powers of the external momenta. We will show that the contact terms in (5.9) result in vanishing contributions and therefore the entire contribution to $\lambda_{3(S)}^{(6)}$ arise from the Wick contractions of the first term in (5.9). We now systematically proceed to evaluate the terms in (B.10) and (B.11) to obtain the contributions to the transport coefficient. The terms in (B.10) and (B.11) can be arranged in polynomials in $p, k$ according to the powers of the external momenta in the numerator of these expressions.

Examining the the terms in (B.10) and (B.11) can be arranged according to decreasing powers of the external momenta in the numerator as follows. The orders in external momenta are also indicated alongside the terms

$$
\begin{aligned}
& M_{1}=\frac{-i\left(p^{b}\right)^{4} p_{3}^{b} p_{3}^{y}}{2\left(i \omega^{2}-E_{-p_{3}}^{2}\right)\left(i \omega^{2}-E_{p+p_{3}}^{2}\right)\left(i \omega^{2}-E_{k+p+p_{3}}^{2}\right)}, \quad O\left(p^{4}\right), \\
& M_{2}=-\frac{\left(-i\left(p_{3}^{a}\right)^{2}-i\left(p_{3}^{b}\right)^{2}-i\left(p_{3}^{z}\right)^{2}\right) k^{y}\left(p^{b}\right)^{3}}{2\left(i \omega^{2}-E_{-p_{3}}^{2}\right)\left(i \omega^{2}-E_{p+p_{3}}^{2}\right)\left(i \omega^{2}-E_{k+p+p_{3}}^{2}\right)}, \quad O\left(p^{3} k\right), \\
& M_{3}=\frac{-i p_{3}^{b} p_{3}^{y}\left(k^{y}\right)^{2} p^{b^{2}}}{2\left(i \omega^{2}-E_{k-p_{3}}^{2}\right)\left(i \omega^{2}-E_{-p_{3}}^{2}\right)\left(i \omega^{2}-E_{p+p_{3}}^{2}\right)}, \quad O\left(p^{2} k^{2}\right), \\
& M_{4}=-\frac{\left(i\left(p_{3}^{a}\right)^{2} p_{3}^{y}+3 i\left(p_{3}^{b}\right)^{2} p_{3}^{y}+i\left(p_{3}^{x}\right)^{2} p_{3}^{y}+i\left(p_{3}^{y}\right)^{3}+i p_{3}^{y}\left(p_{3}^{z}\right)^{2}\right)}{2\left(i \omega^{2}-E_{-p_{3}}^{2}\right)\left(i \omega^{2}-E_{p+p_{3}}^{2}\right)\left(i \omega^{2}-E_{k+p+p_{3}}^{2}\right)}\left(p^{b}\right)^{3}, \quad O\left(p^{3}\right), \\
& M_{5}=-\frac{\left(-3 i\left(p_{3}^{a}\right)^{2} p_{3}^{b}-3 i\left(p_{3}^{b}\right)^{3}+i p_{3}^{b}\left(p_{3}^{\tau}\right)^{2}-2 i p_{3}^{b}\left(p_{3}^{x}\right)^{2}-3 i p_{3}^{b}\left(p_{3}^{z}\right)^{2}\right)}{2\left(i \omega^{2}-E_{-p_{3}}^{2}\right)\left(i \omega^{2}-E_{p+p_{3}}^{2}\right)\left(i \omega^{2}-E_{k+p+p_{3}}^{2}\right)} k^{y}\left(p^{b}\right)^{2} \\
& +\frac{i\left(\left(p_{3}^{a}\right)^{2} p_{3}^{b}+\left(p_{3}^{b}\right)^{3}-p_{3}^{b}\left(p_{3}^{\tau}\right)^{2}+2 p_{3}^{b}\left(p_{3}^{x}\right)^{2}+2 p_{3}^{b}\left(p_{3}^{y}\right)^{2}+p_{3}^{b}\left(p_{3}^{z}\right)^{2}\right)}{2\left(i \omega^{2}-E_{k-p_{3}}^{2}\right)\left(i \omega^{2}-E_{-p_{3}}^{2}\right)\left(i \omega^{2}-E_{p+p_{3}}^{2}\right)} k^{y}\left(p^{b}\right)^{2}, \quad O\left(p^{2} k\right), \\
& M_{6}=\frac{i\left(\left(p_{3}^{b}\right)^{2} p_{3}^{y}-\left(p_{3}^{\tau}\right)^{2} p_{3}^{y}+p_{3}^{y}\left(p_{3}^{z}\right)^{2}\right)}{2\left(i \omega^{2}-E_{-p_{3}}^{2}\right)\left(i \omega^{2}-E_{p+p_{3}}^{2}\right)\left(i \omega^{2}-E_{k+p+p_{3}}^{2}\right)}\left(k^{y}\right)^{2} p^{b} \\
& +\frac{i\left(\left(p_{3}^{a}\right)^{2} p_{3}^{y}+2\left(p_{3}^{b}\right)^{2} p_{3}^{y}+\left(p_{3}^{x}\right)^{2} p_{3}^{y}+\left(p_{3}^{y}\right)^{3}+2 p_{3}^{y}\left(p_{3}^{z}\right)^{2}\right)}{2\left(i \omega^{2}-E_{k-p_{3}}^{2}\right)\left(i \omega^{2}-E_{-p_{3}}^{2}\right)\left(i \omega^{2}-E_{p+p_{3}}^{2}\right)}\left(k^{y}\right)^{2} p^{b}, \quad O\left(p k^{2}\right), \\
& M_{7}=-\frac{\left(3 i\left(p_{3}^{a}\right)^{2} p_{3}^{b} p_{3}^{y}+3 i\left(p_{3}^{b}\right)^{3} p_{3}^{y}+i p_{3}^{b}\left(p_{3}^{\tau}\right)^{2} p_{3}^{y}+3 i p_{3}^{b}\left(p_{3}^{x}\right)^{2} p_{3}^{y}+3 i p_{3}^{b}\left(p_{3}^{y}\right)^{3}+3 i p_{3}^{b} p_{3}^{y}\left(p_{3}^{z}\right)^{2}\right)}{2\left(i \omega^{2}-E_{-p_{3}}^{2}\right)\left(i \omega^{2}-E_{p+p_{3}}^{2}\right)\left(i \omega^{2}-E_{k+p+p_{3}}^{2}\right)}\left(p^{b}\right)^{2} \\
& -\frac{i p_{3}^{b} p_{3}^{y}\left(\left(p_{3}^{a}\right)^{2}+\left(p_{3}^{b}\right)^{2}+\left(p_{3}^{\tau}\right)^{2}+\left(p_{3}^{x}\right)^{2}+\left(p_{3}^{y}\right)^{2}+\left(p_{3}^{z}\right)^{2}\right)}{2\left(i \omega^{2}-E_{k-p_{3}}^{2}\right)\left(i \omega^{2}-E_{-p_{3}}^{2}\right)\left(i \omega^{2}-E_{p+p_{3}}^{2}\right)}\left(p^{b}\right)^{2}, \quad O\left(p^{2}\right),
\end{aligned}
$$




$$
\begin{aligned}
& M_{8}^{A}=i\left[\left(p_{3}^{a}\right)^{4}+3\left(p_{3}^{b}\right)^{4}-\left(3\left(p_{3}^{\tau}\right)^{2}-\left(p_{3}^{x}\right)^{2}+\left(p_{3}^{y}\right)^{2}-\left(p_{3}^{z}\right)^{2}\right)\left(\left(p_{3}^{x}\right)^{2}+\left(p_{3}^{y}\right)^{2}+\left(p_{3}^{z}\right)^{2}\right)+\left(p_{3}^{b}\right)^{2}\right. \\
& \left.\left.\times\left(-\left(p_{3}^{\tau}\right)^{2}+4\left(p_{3}^{x}\right)^{2}+2\left(p_{3}^{y}\right)^{2}+4\left(p_{3}^{z}\right)^{2}\right)+\left(p_{3}^{a}\right)^{2}\left(4\left(p_{3}^{b}\right)^{2}-3\left(p_{3}^{\tau}\right)^{2}+2\left(\left(p_{3}^{x}\right)^{2}+\left(p_{3}^{z}\right)^{2}\right)\right)\right)\right] k^{y} p^{b} \\
& \times \frac{1}{2\left(i \omega^{2}-E_{-p_{3}}^{2}\right)\left(i \omega^{2}-E_{p+p_{3}}^{2}\right)\left(i \omega^{2}-E_{k+p+p_{3}}^{2}\right)}, \\
& M_{8}^{B}=i\left(\left(p_{3}^{a}\right)^{4}+3\left(p_{3}^{a}\right)^{2}\left(p_{3}^{b}\right)^{2}+2\left(p_{3}^{b}\right)^{4}-3\left(p_{3}^{a}\right)^{2}\left(p_{3}^{\tau}\right)^{2}-2\left(p_{3}^{b}\right)^{2}\left(p_{3}^{\tau}\right)^{2}+2\left(p_{3}^{a}\right)^{2}\left(p_{3}^{x}\right)^{2}\right. \\
& +3\left(p_{3}^{b}\right)^{2}\left(p_{3}^{x}\right)^{2}-3\left(p_{3}^{\tau}\right)^{2}\left(p_{3}^{x}\right)^{2}+\left(p_{3}^{x}\right)^{4}+3\left(p_{3}^{a}\right)^{2}\left(p_{3}^{y}\right)^{2}+4\left(p_{3}^{b}\right)^{2}\left(p_{3}^{y}\right)^{2}+3\left(p_{3}^{x}\right)^{2}\left(p_{3}^{y}\right)^{2}+2\left(p_{3}^{y}\right)^{4} \\
& \left.+2\left(p_{3}^{a}\right)^{2}\left(p_{3}^{z}\right)^{2}+3\left(p_{3}^{b}\right)^{2}\left(p_{3}^{z}\right)^{2}-3\left(p_{3}^{\tau}\right)^{2}\left(p_{3}^{z}\right)^{2}+2\left(p_{3}^{x}\right)^{2}\left(p_{3}^{z}\right)^{2}+3\left(p_{3}^{y}\right)^{2}\left(p_{3}^{z}\right)^{2}+\left(p_{3}^{z}\right)^{4}\right) p^{b} k^{y} \\
& \times \frac{1}{2\left(i \omega^{2}-E_{k-p_{3}}^{2}\right)\left(i \omega^{2}-E_{-p_{3}}^{2}\right)\left(i \omega^{2}-E_{p+p_{3}}^{2}\right)}, \quad O(p k), \\
& M_{9}=\frac{i\left(\left(p_{3}^{a}\right)^{2} p_{3}^{b} p_{3}^{y}+\left(p_{3}^{b}\right)^{3} p_{3}^{y}+p_{3}^{b}\left(p_{3}^{\tau}\right)^{2} p_{3}^{y}+p_{3}^{b}\left(p_{3}^{x}\right)^{2} p_{3}^{y}+p_{3}^{b}\left(p_{3}^{y}\right)^{3}+p_{3}^{b} p_{3}^{y}\left(p_{3}^{z}\right)^{2}\right) k^{y^{2}}}{2\left(i \omega^{2}-E_{-p_{3}}^{2}\right)\left(i \omega^{2}-E_{p+p_{3}}^{2}\right)\left(i \omega^{2}-E_{k+p+p_{3}}^{2}\right)} \\
& -\frac{k^{y^{2}} i\left(\left(p_{3}^{a}\right)^{2} p_{3}^{b} p_{3}^{y}+\left(p_{3}^{b}\right)^{3} p_{3}^{y}+p_{3}^{b}\left(p_{3}^{\tau}\right)^{2} p_{3}^{y}+p_{3}^{b}\left(p_{3}^{x}\right)^{2} p_{3}^{y}+p_{3}^{b}\left(p_{3}^{y}\right)^{3}+p_{3}^{b} p_{3}^{y}\left(p_{3}^{z}\right)^{2}\right)}{2\left(i \omega^{2}-E_{k-p_{3}}^{2}\right)\left(i \omega^{2}-E_{-p_{3}}^{2}\right)\left(i \omega^{2}-E_{p+p_{3}}^{2}\right)}, \quad O\left(k^{2}\right), \\
& M_{10}=i p_{3}^{b}\left[\left(p_{3}^{a}\right)^{2}+\left(p_{3}^{b}\right)^{2}-\left(p_{3}^{\tau}\right)^{2}+\left(p_{3}^{x}\right)^{2}+\left(p_{3}^{y}\right)^{2}+\left(p_{3}^{z}\right)^{2}\right]\left[\left(p_{3}^{a}\right)^{2}+\left(p_{3}^{b}\right)^{2}+\left(p_{3}^{\tau}\right)^{2}+\left(p_{3}^{x}\right)^{2}\right. \\
& \left.+\left(p_{3}^{y}\right)^{2}+\left(p_{3}^{z}\right)^{2}\right] \frac{1}{2\left(i \omega^{2}-E_{-p_{3}}^{2}\right)\left(i \omega^{2}-E_{p+p_{3}}^{2}\right)\left(i \omega^{2}-E_{k+p+p_{3}}^{2}\right)} k^{y} \\
& +i p_{3}^{b}\left[\left(p_{3}^{a}\right)^{2}+\left(p_{3}^{b}\right)^{2}-\left(p_{3}^{\tau}\right)^{2}+\left(p_{3}^{x}\right)^{2}+\left(p_{3}^{y}\right)^{2}+\left(p_{3}^{z}\right)^{2}\right]\left[\left(p_{3}^{a}\right)^{2}+\left(p_{3}^{b}\right)^{2}+\left(p_{3}^{\tau}\right)^{2}+\left(p_{3}^{x}\right)^{2}\right. \\
& \left.+\left(p_{3}^{y}\right)^{2}+\left(p_{3}^{z}\right)^{2}\right] \frac{1}{2\left(i \omega^{2}-E_{k-p_{3}}^{2}\right)\left(i \omega^{2}-E_{-p_{3}}^{2}\right)\left(i \omega^{2}-E_{p+p_{3}}^{2}\right)} k^{y}, \quad O(k), \\
& M_{11}=-\frac{i p_{3}^{y}\left(\left(p_{3}^{a}\right)^{2}+\left(p_{3}^{b}\right)^{2}+\left(p_{3}^{x}\right)^{2}+\left(p_{3}^{y}\right)^{2}+\left(p_{3}^{z}\right)^{2}\right)\left(\left(p_{3}^{a}\right)^{2}+\left(p_{3}^{b}\right)^{2}+\left(p_{3}^{\tau}\right)^{2}+\left(p_{3}^{x}\right)^{2}+\left(p_{3}^{y}\right)^{2}+\left(p_{3}^{z}\right)^{2}\right)}{2\left(i \omega^{2}-E_{-p_{3}}^{2}\right)\left(i \omega^{2}-E_{p+p_{3}}^{2}\right)\left(i \omega^{2}-E_{k+p+p_{3}}^{2}\right)} p^{b} \\
& -\frac{i p_{3}^{y}\left(\left(p_{3}^{a}\right)^{2}+\left(p_{3}^{b}\right)^{2}+\left(p_{3}^{x}\right)^{2}+\left(p_{3}^{y}\right)^{2}+\left(p_{3}^{z}\right)^{2}\right)\left(\left(p_{3}^{a}\right)^{2}+\left(p_{3}^{b}\right)^{2}+\left(p_{3}^{\tau}\right)^{2}+\left(p_{3}^{x}\right)^{2}+\left(p_{3}^{y}\right)^{2}+\left(p_{3}^{z}\right)^{2}\right)}{2\left(i \omega^{2}-E_{k-p_{3}}^{2}\right)\left(i \omega^{2}-E_{-p_{3}}^{2}\right)\left(i \omega^{2}-E_{p+p_{3}}^{2}\right)} p^{b}, \\
& O(p),
\end{aligned}
$$

Terms which do not contribute. We now show that all contributions from $M_{1}$ to $M_{7}$ vanish on dividing by $p^{b} k^{y}$, summing over the Matsubara frequencies, performing the angular integrals and then taking the zero momentum limit.

\section{Vanishing of $M_{1}$.}

$$
\begin{aligned}
I_{M_{1}} & =\frac{-1}{p^{b} k^{y}} \sum_{\omega_{m}} \int \frac{d^{5} p_{3}}{(2 \pi)^{5}} \frac{i\left(p^{b}\right)^{4} p_{3}^{b} p_{3}^{y}}{2\left(i \omega^{2}-E_{-p_{3}}^{2}\right)\left(i \omega^{2}-E_{p+p_{3}}^{2}\right)\left(i \omega^{2}-E_{k+p+p_{3}}^{2}\right)}, \\
& =\int \frac{d^{5} p_{3}}{(2 \pi)^{5}} \frac{-i\left(p^{b}\right)^{3} p_{3}^{b} p_{3}^{y}}{2 k^{y}} \mathcal{M} .
\end{aligned}
$$


where we have performed the Matsubara sum using

$$
\begin{aligned}
\mathcal{M} \equiv & \frac{1}{\beta} \sum_{m} \frac{1}{\left(i \omega_{m}^{2}-E_{-p_{3}}^{2}\right)\left(i \omega_{m}^{2}-E_{p+p_{3}}^{2}\right)\left(i \omega_{m}^{2}-E_{k+p+p_{3}}^{2}\right)}, \\
= & \frac{-b\left(E_{p 3+p+k}\right)}{E_{p 3+p+k}\left(E_{p_{3}+p+k}^{2}-E_{p_{3}}^{2}\right)\left(E_{p_{3}+p+k}^{2}-E_{p_{3}+p}^{2}\right)}+\frac{-b\left(E_{p_{3}+p}\right)}{E_{p_{3}+p}\left(E_{p_{3}+p}^{2}-E_{p_{3}+p+k}^{2}\right)\left(E_{p_{3}+p}^{2}-E_{p_{3}}^{2}\right)} \\
& +\frac{-b\left(E_{p_{3}}\right)}{E_{p_{3}}\left(E_{p_{3}}^{2}-E_{p_{3}+p+k}^{2}\right)\left(E_{p_{3}}^{2}-E_{p_{3}+p}^{2}\right)} .
\end{aligned}
$$

We apply the shift of variables $p_{3} \rightarrow-p_{3}-p, p_{3} \rightarrow-p_{3}-p-k$, in $I_{M_{1}}$ to obtain,

$$
\begin{aligned}
I_{M_{1}}= & \frac{-1}{p^{b} k^{y}} \int \frac{d^{5} p_{3}}{(2 \pi)^{5}} \frac{-i\left(p^{b}\right)^{4} p_{3}^{b} p_{3}^{y} b\left(E_{p_{3}}\right)}{2 E_{p_{3}}}\left(\frac{1}{\left(E_{p_{3}}^{2}-E_{p_{3}+p+k}^{2}\right)\left(E_{p_{3}}^{2}-E_{p_{3}+p}^{2}\right)}\right) \\
& \left.\frac{1}{\left(E_{p_{3}}^{2}-E_{p_{3}+p}^{2}\right)\left(E_{p_{3}}^{2}-E_{p_{3}-k}^{2}\right)}+\frac{1}{\left(E_{p_{3}}^{2}-E_{p_{3}+k}^{2}\right)\left(E_{p_{3}}^{2}-E_{p_{3}+p+k}^{2}\right)}\right) \\
& -\int \frac{d^{5} p_{3}}{(2 \pi)^{5}}\left(\frac{-i\left(p^{b}\right)^{4} p_{3}^{y} b\left(E_{p_{3}}\right)}{2 k^{y} E_{p_{3}}\left(E_{p_{3}}^{2}-E_{p_{3}+p}^{2}\right)\left(E_{p_{3}}^{2}-E_{p_{3}-k}^{2}\right)}\right. \\
& \left.+\frac{-i\left(p^{b}\right)^{3}\left(p^{b} p_{3}^{y}+k^{y} p_{3}^{b}+p k\right) b\left(E_{p_{3}}\right)}{2 k E_{p_{3}}\left(E_{p_{3}}^{2}-E_{p_{3}+k}^{2}\right)\left(E_{p_{3}}^{2}-E_{p_{3}+p+k}^{2}\right)}\right)
\end{aligned}
$$

Here $b(p)$ is the Bose-Einstein distribution which is given by

$$
b(p)=\frac{1}{e^{p \beta}-1} .
$$

Subsequently we will require the following moment of the Bose-Einstein distribution at several instances

$$
\begin{aligned}
\int_{0}^{\infty} d p \frac{p^{3}}{e^{\beta p}-1} & =\sum_{n=1}^{\infty} \frac{1}{n^{4} \beta^{4}} \int_{0}^{\infty} d z z^{3} e^{-z} \\
& =\frac{T^{4} \pi^{4}}{15}
\end{aligned}
$$

Let us now take the limit $p^{b} \rightarrow 0, k^{y} \rightarrow 0$ systematically in (B.16). We have verified in all situations the results are independent of the order of limits. For the case of $I_{M_{1}}$ we will demonstrate this explicitly. First we take $k \rightarrow 0$. We obtain the expansion

$$
\lim _{k \rightarrow 0} I_{M_{1}}=\frac{f_{1}(p)}{k}+f_{2}(p)+f_{3}(p) k+O\left(k^{2}\right) \cdots
$$


We denote $p^{b}$ by $p$ and $k^{y}$ by $k$ for convenience. $f_{1}(p)$ and $f_{3}(p)$ are given by

$$
\begin{aligned}
f_{1}(p)= & \int \frac{d^{5} p_{3}}{(2 \pi)^{5}} i b\left(E_{p_{3}}\right)\left(\frac{-E_{p_{3}} p^{2} \cos \phi_{1}}{4\left(E_{p_{3}}^{2} \sin \phi_{1} \cos \phi_{2}\left(p+2 E_{p_{3}} \cos \phi_{1}\right)\right)}\right. \\
& \left.+\frac{p^{3} \cos \phi_{1}+2 p^{2} E_{p_{3}} \cos \phi_{1}^{2}-2 p^{2} E_{p_{3}} \sin \phi_{1} \cos \phi_{2}^{2}}{2 E_{p_{3}} \sin \phi_{1} \cos \phi_{2}\left(p+2 E_{p_{3}} \cos \phi_{1}\right)^{2}}\right), \\
f_{2}(p)= & \int \frac{d^{5} p_{3}}{(2 \pi)^{5}} \frac{i b\left(E_{p_{3}}\right)}{4 E_{p_{3}}^{3} \sin \phi_{1} \cos \phi_{2}^{2}\left(p+2 E_{p_{3}} \cos \phi_{1}\right)^{3}} \\
& \times\left[-p^{5}-5 p^{4} E_{p_{3}} \cos \phi_{1}-8 p^{3} E_{p_{3}}^{2} \cos \phi_{1}^{2}\right. \\
& -4 p^{3} E_{p_{3}}^{2} \sin \phi_{1} \cos \phi_{2}^{2}-4 p^{2} E_{p_{3}}^{3} \cos \phi_{1}^{3}-12 p^{2} E_{p_{3}}^{3} \cos \phi_{1} \sin \phi_{1} \cos \phi_{2}^{2} \\
& \left.-8 p E_{p_{3}}^{4} \cos \phi_{1}^{2} \sin \phi_{1} \cos \phi_{2}^{2}+8 p E_{p_{3}}^{4} \sin \phi_{1} \cos \phi_{2}^{4}\right] .
\end{aligned}
$$

Recall that the integration measure is given by

$$
d^{5} p_{3}=\left|p_{3}\right|^{4} \sin \phi_{1}^{3} \sin \phi_{2}^{2} \sin \phi_{3} d p_{3} .
$$

Note that $f_{1}(p)$ are odd in $\cos \phi_{2}$ where the limits on $\phi_{2}$ run from $0 \rightarrow \pi$. The angular integral over $\phi_{2}$ in $f_{1}(p)$ is of two types. The first one is given by

$$
I_{f_{1}(p)}^{(1)}=\int_{0}^{\pi} \frac{\sin \phi_{2}^{2}}{\cos \phi_{2}}=\int_{-1}^{1} \frac{\sqrt{1-z^{2}}}{z}=0,
$$

where we have used the $i \epsilon$ prescription developed in [16] for evaluating the integral. The second one is given by

$$
I_{f_{1}(p)}^{(2)}=\int_{0}^{\pi} \sin \phi_{2}^{2} \cos \phi_{2}=0 .
$$

The singular term in $k$ given by $f_{1}(p)$ vanished on performing the angular integration. Lets examine $f_{2}(p)$, here we can take the limit $p \rightarrow 0$ and see that it vanishes.

Let us now examine the limit when we first take $p \rightarrow 0$ and then $k \rightarrow 0$. We obtain an expression of the form

$$
\lim _{p \rightarrow 0} I_{M_{1}}=g_{1}(k) p^{2}+g_{2}(k) p^{4}+\cdots
$$

The series has no singular or constant terms in $p$. Thus in this sequence of limits we see $I_{M_{1}}$ tends to zero on taking the first limit. As expected the final result that $I_{M_{1}}$ vanishes is independent of the order of limits. We have verified that this property is true for all the terms in the correlators Similar analysis shows that

$$
\lim _{p \rightarrow 0, k \rightarrow 0} I_{M_{2}}=\lim _{p \rightarrow 0, k \rightarrow 0} I_{M_{3}}=0 .
$$

Vanishing of $\boldsymbol{M}_{\mathbf{4}}$. We show that the integral $M_{M_{4}}$ also vanishes on take the external momenta to zero. Here the mechanism by which it vanishes is different from that of $I_{M_{1}}$ so we discuss it in detail.

$$
I_{M_{4}}=\frac{-1}{p^{b} k^{y}} \sum_{\omega_{m}} \frac{1}{\beta} \int \frac{d^{5} p_{3}}{(2 \pi)^{5}} \frac{\left(i\left(p_{3}^{a}\right)^{2} p_{3}^{y}+3 i\left(p_{3}^{b}\right)^{2} p_{3}^{y}+i\left(p_{3}^{x}\right)^{2} p_{3}^{y}+i\left(p_{3}^{y}\right)^{3}+i p_{3}^{y}\left(p_{3}^{z}\right)^{2}\right)}{2\left(i \omega^{2}-E_{-p_{3}}^{2}\right)\left(i \omega^{2}-E_{p+p_{3}}^{2}\right)\left(i \omega^{2}-E_{k+p+p_{3}}^{2}\right)}\left(p^{b}\right)^{3} .
$$


Lets examine the integral term by term. Consider the second term

$$
\begin{aligned}
I_{M_{4}, 2} & =-\frac{\left(p^{b}\right)^{2}}{k^{y}} \sum_{\omega_{m}} \frac{1}{\beta} \int \frac{d^{5} p_{3}}{(2 \pi)^{5}} \frac{3 i\left(p_{3}^{b}\right)^{2} p_{3}^{y}}{2\left(i \omega^{2}-E_{-p_{3}}^{2}\right)\left(i \omega^{2}-E_{p+p_{3}}^{2}\right)\left(i \omega^{2}-E_{k+p+p_{3}}^{2}\right)}, \\
& =-\int \frac{d^{5} p_{3}}{(2 \pi)^{5}} \frac{3 i p_{3}^{b^{2}} p_{3}^{y}\left(p^{b}\right)^{2}}{k^{y}} \mathcal{M} .
\end{aligned}
$$

We shift the variables to get,

$$
\begin{aligned}
I_{M_{4,2}}= & \int \frac{d^{5} p_{3}}{(2 \pi)^{5}} \frac{3 i\left(p_{3}^{b}\right)^{2} p_{3}^{y} b\left(p_{3}\right)\left(p^{b}\right)^{2}}{E_{p_{3}} k}\left(\frac{-1}{\left(E_{p_{3}}^{2}-E_{p_{3}+p}^{2}\right)\left(E_{p_{3}}^{2}-E_{p_{3}-k}^{2}\right)}\right. \\
& \left.+\frac{-1}{\left(E_{p_{3}}^{2}-E_{p_{3}+k}^{2}\right)\left(E_{p_{3}}^{2}-E_{p_{3}+p+k}^{2}\right)}+\frac{1}{\left(E_{p_{3}}^{2}-E_{p_{3}+p}^{2}\right)\left(E_{p_{3}}^{2}-E_{p_{3}+p+k}^{2}\right)}\right) \\
& -\int \frac{d^{5} p_{3}}{(2 \pi)^{5}} \frac{-i b\left(p_{3}\right) p^{2}}{E_{p_{3}} k} \frac{\left(-6 p_{3}^{b} p_{3}^{y} p-3 p^{2} p_{3}^{y}\right)}{\left(E_{p_{3}}^{2}-E_{p_{3}+p}^{2}\right)\left(E_{p_{3}}^{2}-E_{p_{3}-k}^{2}\right)} \\
& -\frac{-i b\left(p_{3}\right)\left(p^{b}\right)^{2}}{E_{p_{3}} k} \frac{\left(-3 k^{y}\left(p_{3}\right)^{b^{2}}-6 p_{3}^{b} p_{3}^{y} p^{b}-3\left(p^{b}\right)^{2} p_{3}^{y}-6 p_{3}^{b} p^{b} k^{y}+3 k^{y}\left(p^{b}\right) 2\right)}{\left(E_{p_{3}}^{2}-E_{p_{3}+k}^{2}\right)\left(E_{p_{3}}^{2}-E_{p_{3}+p+k}^{2}\right)}
\end{aligned}
$$

We now take $k \rightarrow 0$ first and then $p \rightarrow 0$. There are no singular terms, but there is a finite term which is given by

$$
\begin{aligned}
\lim _{p^{b}, k^{y} \rightarrow 0} I_{M_{4,2}} & =\int \frac{d^{5} p_{3}}{(2 \pi)^{5}} \frac{3 i b\left(E_{p_{3}}\right) \sin ^{2} \phi_{1} \cos ^{2} \phi_{2}}{4 E_{p_{3}} \cos ^{2} \phi_{1}}+\frac{3 i b\left(E_{p_{3}}\right)}{4 E_{p_{3}}}, \\
& =0
\end{aligned}
$$

Thus the putative finite term vanishes on performing the angular integral. Similarly lets examine the other terms in $I_{M_{4}}$.

$$
\begin{aligned}
I_{M_{4,1}} & =\frac{-1}{p^{b} k^{y}} \sum_{\omega_{m}} \frac{1}{\beta} \int \frac{d^{5} p_{3}}{(2 \pi)^{5}} \frac{\left(i\left(p_{3}^{a}\right)^{2} p_{3}^{y}\right)}{2\left(i \omega^{2}-E_{-p_{3}}^{2}\right)\left(i \omega^{2}-E_{p+p_{3}}^{2}\right)\left(i \omega^{2}-E_{k+p+p_{3}}^{2}\right)}\left(p^{b}\right)^{3}, \\
\lim _{p^{b}, k^{y} \rightarrow 0} I_{M_{4,1}} & =-\int \frac{d^{5} p_{3}}{(2 \pi)^{5}} \frac{-3 i p_{3}^{a} \sin ^{2} \phi_{1} \cos ^{2} \phi_{2}}{4 E_{p_{3}} \cos ^{4} \phi_{1}}+\frac{-3 i p_{3}^{a 2}}{4 E_{p_{3}} \cos ^{2} \phi_{1}}, \\
& =0 .
\end{aligned}
$$

Again we have

$$
\begin{aligned}
I_{M_{4,4}} & =\frac{-1}{p^{b} k^{y}} \sum_{\omega_{m}} \frac{1}{\beta} \int \frac{d^{5} p_{3}}{(2 \pi)^{5}} \frac{\left(i\left(p_{3}^{y}\right)^{3}\right)}{2\left(i \omega^{2}-E_{-p_{3}}^{2}\right)\left(i \omega^{2}-E_{p+p_{3}}^{2}\right)\left(i \omega^{2}-E_{k+p+p_{3}}^{2}\right)}\left(p^{b}\right)^{3}, \\
\lim _{p^{b}, k^{y} \rightarrow 0} I_{M_{4,4}} & =-\int \frac{d^{5} p_{3}}{(2 \pi)^{5}} \frac{-3 i b\left(E_{p_{3}}\right) \sin ^{2} \phi_{1} \cos ^{2} \phi_{2}}{4 E_{p_{3}} \cos ^{2} \phi_{1}}+\frac{-3 i b\left(E_{p_{3}}\right) \sin ^{4} \phi_{1} \cos ^{4} \phi_{2}}{4 E_{p_{3}} \cos ^{4} \phi_{1}} \\
& =0 .
\end{aligned}
$$


Therefore we conclude that

$$
I_{M_{4}}=0 .
$$

Vanishing of $\boldsymbol{M}_{\mathbf{6}}$. Writing out $M_{6}$ we have

$$
\begin{aligned}
M_{6}= & \frac{-1}{p^{b} k^{y}} \sum_{\omega_{m}} \frac{1}{\beta} \int \frac{d^{5} p_{3}}{(2 \pi)^{5}}-\frac{i\left(\left(p_{3}^{b}\right)^{2} p_{3}^{y}-\left(p_{3}^{\tau}\right)^{2} p_{3}^{y}+p_{3}^{y}\left(p_{3}^{z}\right)^{2}\right)}{2\left(i \omega^{2}-E_{-p_{3}}^{2}\right)\left(i \omega^{2}-E_{p+p_{3}}^{2}\right)\left(i \omega^{2}-E_{k+p+p_{3}}^{2}\right)}\left(k^{y}\right)^{2} p^{b} \\
& -\frac{i\left(\left(p_{3}^{a}\right)^{2} p_{3}^{y}+2\left(p_{3}^{b}\right)^{2} p_{3}^{y}+\left(p_{3}^{x}\right)^{2} p_{3}^{y}+\left(p_{3}^{y}\right)^{3}+2 p_{3}^{y}\left(p_{3}^{z}\right)^{2}\right)}{2\left(i \omega^{2}-E_{k-p_{3}}^{2}\right)\left(i \omega^{2}-E_{-p_{3}}^{2}\right)\left(i \omega^{2}-E_{p+p_{3}}^{2}\right)}\left(k^{y}\right)^{2} p^{b} .
\end{aligned}
$$

Lets examine the first term

$$
\begin{aligned}
I_{M_{6,1}}= & \frac{\sum_{\omega_{m}}}{\beta} \int \frac{d^{5} p_{3}}{(2 \pi)^{5}} \frac{i\left(k^{y}\left(p_{3}^{b}\right)^{2} p_{3}^{y}\right)}{2\left(i \omega^{2}-E_{-p_{3}}^{2}\right)\left(i \omega^{2}-E_{p+p_{3}}^{2}\right)\left(i \omega^{2}-E_{k+p+p_{3}}^{2}\right)} \\
= & \int \frac{d^{5} p_{3}}{(2 \pi)^{5}} \frac{-i k^{y}\left(p_{3}^{b}\right)^{2} p_{3}^{y} b\left(E_{p_{3}}\right)}{2 E_{p_{3}}}\left(\frac{1}{\left(E_{p_{3}}^{2}-E_{p_{3}+p+k}^{2}\right)\left(E_{p_{3}}^{2}-E_{p_{3}+p}^{2}\right)}\right. \\
& \left.-\frac{1}{\left(E_{p_{3}}^{2}-E_{p_{3}+p}^{2}\right)\left(E_{p_{3}}^{2}-E_{p_{3}-k}^{2}\right)}-\frac{1}{\left(E_{p_{3}}^{2}-E_{p_{3}+k}^{2}\right)\left(E_{p_{3}}^{2}-E_{p_{3}+p+k}^{2}\right)}\right) \\
& -\int \frac{d^{5} p_{3}}{(2 \pi)^{5}} \frac{i k^{y} b\left(E_{p_{3}}\right)}{2 E_{p_{3}}}\left(\frac{\left(-2 p_{3}^{b} p_{3}^{y} p-p^{2} p_{3}^{y}\right)}{\left(E_{p_{3}}^{2}-E_{p_{3}+p}^{2}\right)\left(E_{p_{3}}^{2}-E_{p_{3}-k}^{2}\right)}\right. \\
& +\frac{\left(-2 p_{3}^{b} p_{3}^{y} p-p^{2} p_{3}^{y}-k p_{3}^{b}-2 p k p_{3}^{b}-k p^{2}\right)}{\left(E_{p_{3}}^{2}-E_{p_{3}+k}^{2}\right)\left(E_{p_{3}}^{2}-E_{p_{3}+p+k}^{2}\right)} . \\
\lim _{k^{y} \rightarrow 0} I_{M_{6,1}}= & g_{1}(p) k+g_{2}(p) O\left(k^{2}\right) \cdots
\end{aligned}
$$

Hence in the sequence of limits where we take $k \rightarrow 0$ first, there are no finite terms. This occurs for rest of the terms in $I_{M_{6}}$. In order to establish that this result is independent of the order of limits, we look at the other sequence of limits ( $p \rightarrow 0$ first).

$$
\begin{aligned}
\lim _{k^{y}, p^{b} \rightarrow 0} I_{M_{6,1}} & =-\int \frac{d^{5} p_{3}}{(2 \pi)^{5}} \frac{i b\left(E_{p_{3}}\right)}{2}\left(\frac{1}{4 E_{p_{3}}}+\frac{\cos ^{2} \phi_{1}}{4 E_{p_{3}} \sin ^{2} \phi_{1} \cos ^{2} \phi_{2}}\right), \\
& =0 .
\end{aligned}
$$

similarly,

$$
\begin{aligned}
I_{M_{6,2}} & =\frac{-1}{p^{b} k^{y}} \frac{\sum_{\omega_{m}}}{\beta} \int \frac{d^{5} p_{3}}{(2 \pi)^{5}} \frac{i\left(\left(p_{3}^{\tau}\right)^{2} p_{3}^{y}\right)}{2\left(i \omega^{2}-E_{-p_{3}}^{2}\right)\left(i \omega^{2}-E_{p+p_{3}}^{2}\right)\left(i \omega^{2}-E_{k+p+p_{3}}^{2}\right)} k^{y^{2}} p^{b}, \\
\lim _{k^{y}, p^{b} \rightarrow 0} I_{M_{6,2}} & =\int \frac{d^{5} p_{3}}{(2 \pi)^{5}} \frac{i b\left(E_{p_{3}}\right)}{2}\left(\frac{1}{4 E_{p_{3}} \cos ^{2} \phi_{1}}-\frac{1}{4 E_{p_{3}} \sin ^{2} \phi_{1} \cos ^{2} \phi_{2}}\right), \\
& =0, \\
& =-\lim _{k^{y}, p^{b} \rightarrow 0} I_{M_{6,3} .}
\end{aligned}
$$


The last term in $I_{M_{6}}$ is given by

$$
\begin{aligned}
I_{M_{6,4}} & =\frac{-1}{p^{b} k^{y}} \frac{\sum_{\omega_{m}}}{\beta} \int \frac{d^{5} p_{3}}{(2 \pi)^{5}} \frac{i\left(\left(p_{3}^{a}\right)^{2} p_{3}^{y}\right)}{2\left(i \omega^{2}-E_{k-p_{3}}^{2}\right)\left(i \omega^{2}-E_{-p_{3}}^{2}\right)\left(i \omega^{2}-E_{p+p_{3}}^{2}\right)}\left(k^{y}\right)^{2} p^{b}, \\
\lim _{k^{y}, p^{b} \rightarrow 0} I_{M_{6,4}} & =-\int \frac{d^{5} p_{3}}{(2 \pi)^{5}}\left(\frac{p_{3}^{a 2}}{4 p_{3}^{3} \cos ^{2} \phi_{1}}-\frac{p_{3}^{a 2}}{4 p_{3}^{3} \sin ^{2} \phi_{1} \cos ^{2} \phi_{2}}\right), \\
& =0 .
\end{aligned}
$$

Therefore we conclude that

$$
I_{M_{6}}=0 .
$$

$I_{M_{5}}$ exhibits and similar mechanism for cancellation, performing the same analysis shows

$$
I_{M_{5}}=0 .
$$

Vanishing of $M_{7}$. Again we analyse $I_{M_{7}}$ term by term to show that it vanishes on taking the external momenta to zero.

$$
\begin{aligned}
I_{M_{7}}= & \frac{-1}{p^{b} k^{y}} \sum_{\omega_{m}} \frac{1}{\beta} \int \frac{d^{5} p_{3}}{(2 \pi)^{5}}\left[3 i\left(p_{3}^{a}\right)^{2} p_{3}^{b} p_{3}^{y}+3 i\left(p_{3}^{b}\right)^{3} p_{3}^{y}+i p_{3}^{b}\left(p_{3}^{\tau}\right)^{2} p_{3}^{y}+3 i p_{3}^{b}\left(p_{3}^{x}\right)^{2} p_{3}^{y}+3 i p_{3}^{b}\left(p_{3}^{y}\right)^{3}\right. \\
& \left.+3 i p_{3}^{b} p_{3}^{y}\left(p_{3}^{z}\right)^{2}\right]\left(p^{b}\right)^{2} \times \frac{1}{2\left(i \omega^{2}-E_{-p_{3}}^{2}\right)\left(i \omega^{2}-E_{p+p_{3}}^{2}\right)\left(i \omega^{2}-E_{k+p+p_{3}}^{2}\right)} \\
& +\frac{-1}{p^{b} k^{y}} \sum_{\omega_{m}} \frac{1}{\beta} \int \frac{d^{5} p_{3}}{(2 \pi)^{5}} \frac{i p_{3}^{b} p_{3}^{y}\left(\left(p_{3}^{a}\right)^{2}+\left(p_{3}^{b}\right)^{2}+\left(p_{3}^{\tau}\right)^{2}+\left(p_{3}^{x}\right)^{2}+\left(p_{3}^{y}\right)^{2}+\left(p_{3}^{z}\right)^{2}\right)}{2\left(i \omega^{2}-E_{k-p_{3}}^{2}\right)\left(i \omega^{2}-E_{-p_{3}}^{2}\right)\left(i \omega^{2}-E_{p+p_{3}}^{2}\right)}\left(p^{b}\right)^{2} .
\end{aligned}
$$

Lets consider the first term

$$
\begin{aligned}
I_{M_{7,1}}= & \frac{-1}{p^{b} k^{y}} \sum_{\omega_{m}} \frac{1}{\beta} \int \frac{d^{5} p_{3}}{(2 \pi)^{5}} \frac{\left(3 i\left(p_{3}^{a}\right)^{2} p_{3}^{b} p_{3}^{y}\right)\left(p^{b}\right)^{2}}{2\left(i \omega^{2}-E_{-p_{3}}^{2}\right)\left(i \omega^{2}-E_{p+p_{3}}^{2}\right)\left(i \omega^{2}-E_{k+p+p_{3}}^{2}\right)}, \\
= & \int \frac{d^{5} p_{3}}{(2 \pi)^{5}} \frac{i p_{3}^{a} p_{3}^{b} p_{3}^{y} p^{b}\left(p_{3}\right)}{2 k E_{p_{3}}}\left(\frac{1}{\left(E_{p_{3}}^{2}-E_{p_{3}+p+k}^{2}\right)\left(E_{p_{3}}^{2}-E_{p_{3}+p}^{2}\right)}\right) \\
& \left.+\frac{1}{\left(E_{p_{3}}^{2}-E_{p_{3}+p}^{2}\right)\left(E_{p_{3}}^{2}-E_{p_{3}-k}^{2}\right)}+\frac{1}{\left(E_{p_{3}}^{2}-E_{p_{3}+k}^{2}\right)\left(E_{p_{3}}^{2}-E_{p_{3}+p+k}^{2}\right)}\right) \\
& +\int \frac{d^{5} p_{3}}{(2 \pi)^{5}} \frac{i p^{2} p_{3}^{a 2} p_{3}^{y} b\left(p_{3}\right)}{2 k E_{p_{3}}} \frac{1}{\left(E_{p_{3}}^{2}-E_{p_{3}+p}^{2}\right)\left(E_{p_{3}}^{2}-E_{p_{3}-k}^{2}\right)} \\
& +\frac{i p_{3}^{a 2} b\left(p_{3}\right)}{2 E_{p_{3}}} \frac{\left(\frac{\left(p^{b}\right)^{2} p_{3}^{y}}{k^{y}}+p^{b} p_{3}^{b}+\left(p^{b}\right)^{2}\right)}{\left(E_{p_{3}}^{2}-E_{p_{3}+k}^{2}\right)\left(E_{p_{3}}^{2}-E_{p_{3}+p+k}^{2}\right)}, \\
\lim _{p^{b}, k^{y} \rightarrow 0} I_{M_{7,1}}= & \int \frac{d^{5} p_{3}}{(2 \pi)^{5}} \frac{i p_{3}^{a 2} b\left(p_{3}\right)}{2}\left(-\frac{1}{8 E_{p_{3}}^{3} \sin ^{2} \phi_{1} \cos ^{2} \phi_{2}}-\frac{3 \sin ^{2} \phi_{1} \cos ^{2} \phi_{2}}{8 E_{p_{3}}^{3} \cos ^{4} \phi_{1}}\right), \\
= & 0 .
\end{aligned}
$$


As a check we also evaluate the alternative sequence of limits, $k \rightarrow 0, p \rightarrow 0$. We find that,

$$
\lim _{k^{y}, p^{b} \rightarrow 0} I_{M_{7,1}}=\frac{g_{3}(p)}{k}+O(p)+O(k) \cdots
$$

Where the angular integral in $g_{3}(p)$ vanishes in a similar mechanism to B.20. Thus this term vanishes irrespective of the order of limits.

Similarly we have analysed all terms in $I_{M_{7}}$ to conclude

$$
I_{M_{7}}=0 .
$$

\section{B.1 Evaluating the non-vanishing contributions}

We now evaluate the finite contribution to the transport coefficient which are given by $M_{8}^{A}, M_{8}^{B}, M_{10}$ and $M_{11}$.

\section{Evaluation of $M_{8}^{A}$ and $M_{8}^{B}$.}

$$
\begin{aligned}
I_{M_{8}}^{A}= & \frac{i}{p^{b} k^{y}} \sum_{\omega_{m}} \frac{1}{\beta} \int \frac{d^{5} p_{3}}{(2 \pi)^{5}}\left[\left(p_{3}^{a}\right)^{4}+3\left(p_{3}^{b}\right)^{4}-\left(3\left(p_{3}^{\tau}\right)^{2}-\left(p_{3}^{x}\right)^{2}+\left(p_{3}^{y}\right)^{2}-\left(p_{3}^{z}\right)^{2}\right)\left(\left(p_{3}^{x}\right)^{2}+\left(p_{3}^{y}\right)^{2}\right.\right. \\
& \left.+\left(p_{3}^{z}\right)^{2}\right)+\left(p_{3}^{b}\right)^{2}\left(-\left(p_{3}^{\tau}\right)^{2}+4\left(p_{3}^{x}\right)^{2}+2\left(p_{3}^{y}\right)^{2}+4\left(p_{3}^{z}\right)^{2}\right)+\left(p_{3}^{a}\right)^{2}\left(4\left(p_{3}^{b}\right)^{2}-3\left(p_{3}^{\tau}\right)^{2}\right. \\
& \left.\left.+2\left(\left(p_{3}^{x}\right)^{2}+\left(p_{3}^{z}\right)^{2}\right)\right)\right] k^{y} p^{b} \times \frac{1}{2\left(i \omega^{2}-E_{-p_{3}}^{2}\right)\left(i \omega^{2}-E_{p+p_{3}}^{2}\right)\left(i \omega^{2}-E_{k+p+p_{3}}^{2}\right)} .
\end{aligned}
$$

We now analyse the integral term by term. We label the terms by the monomial of the internal momentum in the numerator

$$
\begin{aligned}
I_{p_{3}^{a 2} p_{3}^{\tau 2}}^{A}= & \frac{-1}{p^{b} k^{y}} \frac{\sum_{\omega_{m}}}{\beta} \int \frac{d^{5} p_{3}}{(2 \pi)^{5}} \frac{i p_{3}^{a 2} p_{3}^{\tau 2} p^{b} k^{y}}{2\left(i \omega^{2}-E_{-p_{3}}^{2}\right)\left(i \omega^{2}-E_{p+p_{3}}^{2}\right)\left(i \omega^{2}-E_{k+p+p_{3}}^{2}\right)}, \\
= & \frac{i}{2} \int \frac{d^{5} p_{3}}{(2 \pi)^{5}} p_{3}^{a 2} \frac{(i \omega)^{2}}{\left(i \omega^{2}-E_{-p_{3}}^{2}\right)\left(i \omega^{2}-E_{p+p_{3}}^{2}\right)\left(i \omega^{2}-E_{k+p+p_{3}}^{2}\right)}, \\
= & \frac{-i}{2} \int \frac{d^{5} p_{3}}{(2 \pi)^{5}} p_{3}^{a 2}\left(\frac{E_{p_{3}} b\left(E_{p_{3}}\right)}{\left(E_{p_{3}}^{2}-E_{p_{3}+p+k}^{2}\right)\left(E_{p_{3}}^{2}-E_{p_{3}+p}^{2}\right)}\right. \\
& \left.+\frac{E_{p_{3}+p} b\left(E_{p_{3}+p}\right)}{\left(E_{p_{3}+p}^{2}-E_{p_{3}+p+k}^{2}\right)\left(E_{p_{3}+p}^{2}-E_{p_{3}}^{2}\right)}+\frac{E_{p_{3}+p+k} b\left(E_{p_{3}+p+k}\right)}{\left(E_{p_{3}+p+k}^{2}-E_{p_{3}}^{2}\right)\left(E_{p_{3}+p+k}^{2}-E_{p_{3}+p}^{2}\right)}\right) .
\end{aligned}
$$

We perform the usual shift of variables to get,

$$
\lim _{p^{b}, k^{y} \rightarrow 0} I_{p_{3}^{a} p_{3}^{\tau 2}}^{A}=\frac{-i}{2} \int \frac{d p_{3}}{(2 \pi)^{5}} \frac{p_{3}^{3} b\left(p_{3}\right) \sin ^{5} \phi_{1} \sin ^{4} \phi_{2} \sin ^{3} \phi_{3} \sin ^{2} \phi_{4}}{8 \cos ^{2} \phi_{1} \sin ^{2} \phi_{1} \cos ^{2} \phi_{2}} .
$$


After a change of variables $z=\cos \theta$, the required integrals are of the form

$$
J_{1}=\int_{0}^{\pi} \frac{\sin ^{3} \theta d \theta}{\cos ^{2} \theta}=\int_{-1}^{1} \frac{\left(1-z^{2}\right) d z}{z^{2}}, \quad J_{2}=\int_{0}^{\pi} \frac{\sin ^{4} \theta d \theta}{\cos ^{2} \theta}=\int_{-1}^{1} \frac{\left(1-z^{2}\right)^{\frac{3}{2}} d z}{z^{2}} .
$$

The integrals are all on the real line, we make these integrals well defined by slightly deforming the contour to avoid the singularity at $z=0$. This prescription was developed and tested in [16]. Then we obtain

$$
J_{1}=-4, \quad J_{2}=-\frac{3 \pi}{2} .
$$

and using these results for the integrals we get

$$
\lim _{p \rightarrow 0, k \rightarrow 0} I_{p_{3}^{a 2} p_{3}^{\tau 2}}^{A}=\frac{-i \pi T^{4}}{2 \times 15 \times 32} .
$$

Proceeding similarly we arrive at the following relations

$$
I_{p_{3}^{a 2} p_{3}^{\tau 2}}^{A}=I_{p_{3}^{x 2} p_{3}^{\tau 2}}^{A}=I_{p_{3}^{z 2} p_{3}^{\tau 2}}^{A} .
$$

Now consider $I_{-p_{3}^{x 2} p_{3}^{a 2}}^{A}$ and the rest of the terms

$$
\begin{aligned}
I_{-p_{3}^{x} p_{3}^{a 2}}^{A} & =\frac{-1}{p^{b} k^{y}} \sum_{\omega_{m}} \frac{1}{\beta} \int \frac{d^{5} p_{3}}{(2 \pi)^{5}} \frac{-i p_{3}^{a 2} p_{3}^{\tau 2} p^{b} k^{y}}{2\left(i \omega^{2}-E_{-p_{3}}^{2}\right)\left(i \omega^{2}-E_{p+p_{3}}^{2}\right)\left(i \omega^{2}-E_{k+p+p_{3}}^{2}\right)} \\
\lim _{p^{b}, k^{y} 0} I_{-p_{3}^{x 2} p_{3}^{a 2}}^{A} & =\frac{-i}{2} \int \frac{d p_{3}}{(2 \pi)^{5}} \frac{p_{3}^{3} b\left(p_{3}\right) \sin ^{5} \phi_{1} \sin ^{6} \phi_{2} \sin ^{3} \phi_{3} \cos ^{2} \phi_{3} \sin ^{2} \phi_{4}}{8 \cos ^{2} \phi_{1} \cos ^{2} \phi_{2}} \\
& =\frac{-i \pi T^{4}}{2 \times 3 \times 15 \times 32} .
\end{aligned}
$$

where we have used,

$$
\begin{aligned}
& J_{3}=\int_{0}^{\pi} \frac{\sin ^{5} \theta d \theta}{\cos ^{2} \theta}=\int_{-1}^{1} \frac{\left(1-z^{2}\right)^{2} d z}{z^{2}}=\frac{-16}{3}, \\
& J_{4}=\int_{0}^{\pi} \frac{\sin ^{6} \theta d \theta}{\cos ^{2} \theta}=\int_{-1}^{1} \frac{\left(1-z^{2}\right)^{\frac{5}{2}} d z}{z^{2}}=\frac{-15}{8} .
\end{aligned}
$$

Similarly in the zero external momenta limit we get

$$
\begin{aligned}
I_{-p_{3}^{x 2} p_{3}^{a 2}}^{A} & =I_{-p_{3}^{x 2} p_{3}^{z^{2}}}^{A}=I_{-p_{3}^{z^{2}} p_{3}^{a 2}}^{A}=I_{-p_{3}^{x 2} p_{3}^{y 2}}^{A}=I_{-p_{3}^{x^{2}} p_{3}^{b^{2}}}^{A}, \\
& =I_{-p_{3}^{a 2} p_{3}^{y^{2}}}^{A}=I_{-p_{3}^{a 2} p_{3}^{b^{2}}}^{A}=I_{-p_{3}^{b^{2}} p_{3}^{y^{2}}}^{A}=I_{-p_{3}^{z^{2}} p_{3}^{y 2}}^{A}=I_{-p_{3}^{z 2} p_{3}^{b^{2}}}^{A}
\end{aligned}
$$

We then evaluate terms corresponding to $M_{8}^{B}$. We obtain the following results in the zero external momenta limit

$$
\begin{aligned}
I_{p_{3}^{a 2} p_{3}^{\tau^{2}}}^{A} & =I_{p_{3}^{a 2} p_{3}^{\tau^{2}}}^{B}=I_{p_{3}^{x 2} p_{3}^{\tau 2}}^{B}=I_{p_{3}^{z^{2}} p_{3}^{\tau 2}}^{B}=\frac{-i \pi T^{4}}{2 \times 15 \times 32} . \\
I_{-p_{3}^{x 2} p_{3}^{a 2}}^{A} & =I_{-p_{3}^{x 2} p_{3}^{a 2}}^{B}=I_{-p_{3}^{x 2} p_{3}^{z 2}}^{B}=I_{-p_{3}^{z^{2}} p_{3}^{a 2}}^{B}=I_{-p_{3}^{x 2} p_{3}^{y 2}}^{B}=I_{-p_{3}^{x 2} p_{3}^{b^{2}}}^{B} \\
& =I_{-p_{3}^{a 2} p_{3}^{y 2}}^{B}=I_{-p_{3}^{a} p_{3}^{b^{2}}}^{B}=I_{-p_{3}^{b^{2}} p_{3}^{y 2}}^{B}=I_{-p_{3}^{z 2} p_{3}^{y 2}}^{B}=I_{-p_{3}^{z^{2}} p_{3}^{b^{2}}}^{B}=\frac{-i \pi T^{4}}{2 \times 3 \times 15 \times 32} .
\end{aligned}
$$


Substituting these values into the terms of $I_{9}^{A}$ and $M_{M_{9}}^{B}$, we obtain

$$
\lim _{p^{b}, k^{y} \rightarrow 0}\left(I_{M_{9}}^{A}+I_{M_{9}}^{B}\right)=\frac{-18 i \pi T^{4}}{15 \times 32}+\frac{-48 i \pi T^{4}}{6 \times 15 \times 32} .
$$

Evaluation of $\boldsymbol{M}_{\mathbf{1 0}}$ and $\boldsymbol{M}_{\mathbf{1 1}}$. Lets first write down the integrals involving $M_{10}$ and $M_{11}$

$$
\begin{aligned}
I_{M_{10}}= & \frac{-1}{p^{b} k^{y}} \sum_{\omega_{m}} \frac{1}{\beta} \int \frac{d^{5} p_{3}}{(2 \pi)^{5}}\left\{\left(-i p_{3}^{b}\right)\left[\left(p_{3}^{a}\right)^{2}+\left(p_{3}^{b}\right)^{2}-\left(p_{3}^{\tau}\right)^{2}+\left(p_{3}^{x}\right)^{2}+\left(p_{3}^{y}\right)^{2}+\left(p_{3}^{z}\right)^{2}\right]\right. \\
& \times\left[\left(p_{3}^{a}\right)^{2}+\left(p_{3}^{b}\right)^{2}+\left(p_{3}^{\tau}\right)^{2}+\left(p_{3}^{x}\right)^{2}+\left(p_{3}^{y}\right)^{2}+\left(p_{3}^{z}\right)^{2}\right] \frac{k^{y}}{2\left(i \omega^{2}-E_{-p_{3}}^{2}\right)\left(i \omega^{2}-E_{p+p_{3}}^{2}\right)\left(i \omega^{2}-E_{k+p+p_{3}}^{2}\right)} \\
& -i p_{3}^{b}\left[\left(p_{3}^{a}\right)^{2}+\left(p_{3}^{b}\right)^{2}-\left(p_{3}^{\tau}\right)^{2}+\left(p_{3}^{x}\right)^{2}+\left(p_{3}^{y}\right)^{2}+\left(p_{3}^{z}\right)^{2}\right]\left[\left(p_{3}^{a}\right)^{2}+\left(p_{3}^{b}\right)^{2}+\left(p_{3}^{\tau}\right)^{2}+\left(p_{3}^{x}\right)^{2}+\left(p_{3}^{y}\right)^{2}+\left(p_{3}^{z}\right)^{2}\right] \\
& \left.\times \frac{k^{y}}{2\left(i \omega^{2}-E_{k-p_{3}}^{2}\right)\left(i \omega^{2}-E_{-p_{3}}^{2}\right)\left(i \omega^{2}-E_{p+p_{3}}^{2}\right)}\right\} . \\
I_{M_{11}}= & \frac{-1}{p^{b} k^{y}} \sum_{\omega_{m}} \frac{1}{\beta} \int \frac{d^{5} p_{3}}{(2 \pi)^{5}}\left\{i p_{3}^{y}\left[\left(p_{3}^{a}\right)^{2}+\left(p_{3}^{b}\right)^{2}+\left(p_{3}^{x}\right)^{2}+\left(p_{3}^{y}\right)^{2}+\left(p_{3}^{z}\right)^{2}\right]\right. \\
& \times\left[\left(p_{3}^{a}\right)^{2}+\left(p_{3}^{b}\right)^{2}+\left(p_{3}^{\tau}\right)^{2}+\left(p_{3}^{x}\right)^{2}+\left(p_{3}^{y}\right)^{2}+\left(p_{3}^{z}\right)^{2}\right] \frac{p^{b}}{2\left(i \omega^{2}-E_{-p_{3}}^{2}\right)\left(i \omega^{2}-E_{p+p_{3}}^{2}\right)\left(i \omega^{2}-E_{k+p+p_{3}}^{2}\right)} \\
& +\frac{i p_{3}^{y} p^{b}\left[\left(p_{3}^{a}\right)^{2}+\left(p_{3}^{b}\right)^{2}+\left(p_{3}^{x}\right)^{2}+\left(p_{3}^{y}\right)^{2}+\left(p_{3}^{z}\right)^{2}\right]\left[\left(p_{3}^{a}\right)^{2}+\left(p_{3}^{b}\right)^{2}+\left(p_{3}^{\tau}\right)^{2}+\left(p_{3}^{x}\right)^{2}+\left(p_{3}^{y}\right)^{2}+\left(p_{3}^{z}\right)^{2}\right]}{2\left(i \omega^{2}-E_{k-p_{3}}^{2}\right)\left(i \omega^{2}-E_{-p_{3}}^{2}\right)\left(i \omega^{2}-E_{p+p_{3}}^{2}\right)} .
\end{aligned}
$$

We evaluate these contributions in a similar manner as before, summing over Matsubara frequencies and shifting momenta as required. We take the zero momentum limit to get the finite contribution. Lets detail some of the steps by considering generic terms. Consider the integral

$$
\begin{aligned}
\hat{I}_{p_{3}^{a} p_{3}^{b^{2}}}= & \frac{-1}{p^{b} k^{y}} \sum_{\omega_{m}} \frac{1}{\beta} \int \frac{d^{5} p_{3}}{(2 \pi)^{5}} p_{3}^{a 2} p_{3}^{b^{2}}\left(-p_{3}^{b} k^{y}+p_{3}^{y} p^{b}\right) \\
& \times\left(\frac{1}{2\left(i \omega^{2}-E_{-p_{3}}^{2}\right)\left(i \omega^{2}-E_{p+p_{3}}^{2}\right)\left(i \omega^{2}-E_{k+p+p_{3}}^{2}\right)}\right) \\
& \left.+\frac{1}{2\left(i \omega^{2}-E_{k-p_{3}}^{2}\right)\left(i \omega^{2}-E_{-p_{3}}^{2}\right)\left(i \omega^{2}-E_{p+p_{3}}^{2}\right)}\right) \\
= & \hat{I}_{p_{3}^{a 2} p_{3}^{b^{2}}}^{C C}+\hat{I}_{p_{3}^{a} p_{3}^{b^{2}}}^{D}
\end{aligned}
$$

Note we have labelled the integral by the common directions of the internal momenta $\left(p_{3}^{a} p_{3}^{b}\right)^{2}$, of the integrand. We have suppressed by the dependence $\left(-p_{3}^{b} k^{y}+p_{3}^{y} p^{b}\right)$. This will be understood in the remaining integrals of this section which have the superscript ${ }^{\wedge}$. 
We now define

$$
\begin{aligned}
\hat{I}_{p_{3}^{a} p_{3}^{b^{2}}}^{\prime C}= & \frac{-1}{p^{b} k^{y}} \sum_{\omega_{m}} \frac{1}{\beta} \int \frac{d^{5} p_{3}}{(2 \pi)^{5}}\left(p_{3}^{a}\right)^{2}\left(p_{3}^{b}\right)^{2}\left(-p_{3}^{b} k^{y}\right) \\
& \times\left(\frac{1}{2\left(i \omega^{2}-E_{-p_{3}}^{2}\right)\left(i \omega^{2}-E_{p+p_{3}}^{2}\right)\left(i \omega^{2}-E_{k+p+p_{3}}^{2}\right)}\right) \\
& \left.+\frac{1}{2\left(i \omega^{2}-E_{k-p_{3}}^{2}\right)\left(i \omega^{2}-E_{-p_{3}}^{2}\right)\left(i \omega^{2}-E_{p+p_{3}}^{2}\right)}\right), \\
= & \int \frac{d^{5} p_{3}}{(2 \pi)^{5}} \frac{i p_{3}^{a 2} p_{3}^{b^{3}}}{2 p^{b}}\left(\mathcal{M}+\mathcal{M}^{\prime}\right), \\
\hat{I}_{p_{3}^{a 2} p_{3}^{b^{2}}}^{\prime D}= & \int \frac{d^{5} p_{3}}{(2 \pi)^{5}} \frac{-i p_{3}^{a} p_{3}^{b^{2}} p_{3}^{y}}{2 k^{y}}\left(\mathcal{M}+\mathcal{M}^{\prime}\right) .
\end{aligned}
$$

We have used the following formulae to perform the Matsubara sums,

$$
\begin{aligned}
\mathcal{M}= & \frac{1}{\beta} \sum_{m} \frac{1}{\left(i \omega_{m}^{2}-E_{-p_{3}}^{2}\right)\left(i \omega_{m}^{2}-E_{p+p_{3}}^{2}\right)\left(i \omega_{m}^{2}-E_{k+p+p_{3}}^{2}\right)}, \\
= & \frac{-b\left(E_{p 3+p+k}\right)}{E_{p 3+p+k}\left(E_{p_{3}+p+k}^{2}-E_{p_{3}}^{2}\right)\left(E_{p_{3}+p+k}^{2}-E_{p_{3}+p}^{2}\right)} \\
& +\frac{-b\left(E_{p_{3}+p}\right)}{E_{p_{3}+p}\left(E_{p_{3}+p}^{2}-E_{p_{3}+p+k}^{2}\right)\left(E_{p_{3}+p}^{2}-E_{p_{3}}^{2}\right)} \\
& +\frac{-b\left(E_{p_{3}}\right)}{E_{p_{3}}\left(E_{p_{3}}^{2}-E_{p_{3}+p+k}^{2}\right)\left(E_{p_{3}}^{2}-E_{p_{3}+p}^{2}\right)} .
\end{aligned}
$$

and

$$
\begin{aligned}
\mathcal{M}^{\prime}= & \frac{1}{\beta} \sum_{m} \frac{1}{\left(i \omega_{m}^{2}-E_{k-p_{3}}^{2}\right)\left(i \omega_{m}^{2}-E_{p_{3}}^{2}\right)\left(i \omega_{m}^{2}-E_{p+p_{3}}^{2}\right)}, \\
& =\frac{-b\left(E_{p 3}\right)}{E_{p_{3}}\left(E_{p_{3}}^{2}-E_{p_{3}-k}^{2}\right)\left(E_{p_{3}}^{2}-E_{p_{3}+p}^{2}\right)}+\frac{-b\left(E_{p_{3}+p}\right)}{E_{p_{3}+p}\left(E_{p_{3}+p}^{2}-E_{p_{3}-k}^{2}\right)\left(E_{p_{3}+p}^{2}-E_{p_{3}}^{2}\right)} \\
& +\frac{-b\left(E_{p_{3}-k}\right)}{E_{p_{3}-k}\left(E_{p_{3}-k}^{2}-E_{p_{3}+p}^{2}\right)\left(E_{p_{3}-k}^{2}-E_{p_{3}}^{2}\right)} .
\end{aligned}
$$

We shift the variables such that numerator in the each of the terms in the Matsubara sums is a function of $E_{p_{3}}$. For example we shift the variables $p_{3} \rightarrow-p_{3}-p-k$ in the first term 
of $\mathcal{M}$ and so on. Applying such shifts we are left with,

$$
\begin{aligned}
& \hat{I}_{p_{3}^{a} p_{3}^{b^{2}}}^{C C}=\int \frac{d^{5} p_{3}}{(2 \pi)^{5}} \frac{-i p_{3}^{a 2} p_{3}^{b^{3}} b\left(p_{3}\right)}{2 p^{b} E_{p_{3}}}\left(\frac{1}{\left(E_{p_{3}}^{2}-E_{p_{3}-p-k}^{2}\right)\left(E_{p_{3}}^{2}-E_{p_{3}-k}^{2}\right)}\right. \\
& \left.-\frac{1}{\left(E_{p_{3}}^{2}-E_{p_{3}+p}^{2}\right)\left(E_{p_{3}}^{2}-E_{p_{3}-k}^{2}\right)}\right) \\
& +\int \frac{d^{5} p_{3}}{(2 \pi)^{5}} \frac{p_{3}^{a 2}}{p E_{p_{3}}}\left(3 p_{3}^{b^{2}} p+3 p_{3}^{b} p^{2}+p^{3}\right)\left(\frac{1}{\left(E_{p_{3}}^{2}-E_{p_{3}+p+k}^{2}\right)\left(E_{p_{3}}^{2}-E_{p_{3}+p}^{2}\right)}\right. \\
& \left.+\frac{1}{\left(E_{p_{3}}^{2}-E_{p_{3}+p}^{2}\right)\left(E_{p_{3}}^{2}-E_{p_{3}-k}^{2}\right)}+\frac{1}{\left(E_{p_{3}}^{2}-E_{p_{3}+k}^{2}\right)\left(E_{p_{3}}^{2}-E_{p_{3}+p+k}^{2}\right)}\right), \\
& \lim _{p^{b}, k^{y} \rightarrow 0} \hat{I}_{p_{3}^{a 2} p_{3}^{b 2}}^{\prime C}=\int \frac{d^{5} p_{3}}{(2 \pi)^{5}} \frac{-3 i p_{3}^{a} b\left(p_{3}\right)}{8 p_{3}^{3} \sin ^{2} \phi_{1} \cos ^{2} \phi_{2}}, \\
& =\frac{i \pi T^{4}}{2 \times 15 \times 32} \text {. }
\end{aligned}
$$

Following similar steps as done for the above integral we have

$$
\begin{aligned}
\lim _{p^{b}, k^{y} \rightarrow 0} \hat{I}_{p_{3}^{a 2} p_{3}^{b^{2}}}^{D} & =-\int \frac{d^{5} p_{3}}{(2 \pi)^{5}} \frac{i p_{3}^{a 2} b\left(p_{3}\right)}{2}\left(\frac{1}{4 p_{3}^{3} \cos ^{2} \phi_{1}}-\frac{1}{4 p_{3}^{3} \sin ^{2} \phi_{1} \cos ^{2} \phi_{2}}\right), \\
& =0 .
\end{aligned}
$$

From (B.61), (B.65) and (B.66) we have,

$$
\hat{I}_{p_{3}^{a} p_{3}^{b^{2}}}=\frac{i \pi T^{4}}{2 \times 15 \times 32} .
$$

Using similar manipulations, it can be shown that

$$
\begin{aligned}
\hat{I}_{p_{3}^{a 2} p_{3}^{b^{2}}}= & \hat{I}_{p_{3}^{x 2} p_{3}^{b^{2}}}=\hat{I}_{p_{3}^{y 2} p_{3}^{b^{2}}}=\hat{I}_{p_{3}^{z 2} p_{3}^{b^{2}}} . \\
\hat{I}_{p_{3}^{a 2} p_{3}^{x 2}}= & \frac{i \pi T^{4}}{2 \times 3 \times 15 \times 32} . \\
\hat{I}_{p_{3}^{a 2} p_{3}^{y 2}}= & \hat{I}_{p_{3}^{a 2} p_{3}^{z 2}}=\hat{I}_{p_{3}^{y 2} p_{3}^{x 2}}=\hat{I}_{p_{3}^{y 2} p_{3}^{z^{2}}}=\hat{I}_{p_{3}^{x 2} p_{3}^{z 2}}=\hat{I}_{p_{3}^{a 2} p_{3}^{b^{2}}} . \\
I_{p_{3}^{\tau 4}}^{\prime}= & \frac{-1}{p^{b} k^{y}} \sum_{\omega_{m}} \frac{1}{\beta} \int \frac{d^{5} p_{3}}{(2 \pi)^{5}}\left(\left(p_{3}^{\tau}\right)^{4} p_{3}^{b} k^{y}+\left(p_{3}^{\tau}\right)^{2}\left(\left(p_{3}^{a}\right)^{2}+\left(p_{3}^{b}\right)^{2}+\left(p_{3}^{y}\right)^{2}+\left(p_{3}^{x}\right)^{2}+\left(p_{3}^{z}\right)^{2}\right) p_{3}^{y} p^{b}\right) \\
& \times\left(\frac{1}{2\left(i \omega^{2}-E_{-p_{3}}^{2}\right)\left(i \omega^{2}-E_{p+p_{3}}^{2}\right)\left(i \omega^{2}-E_{k+p+p_{3}}^{2}\right)}\right) \\
& \left.+\frac{1}{2\left(i \omega^{2}-E_{k-p_{3}}^{2}\right)\left(i \omega^{2}-E_{-p_{3}}^{2}\right)\left(i \omega^{2}-E_{p+p_{3}}^{2}\right)}\right) \\
= & I_{p_{3}^{\tau 4} C}^{\prime}+I_{p_{3}^{\tau} D^{\prime}}^{\prime}
\end{aligned}
$$


where,

$$
\begin{aligned}
I_{p_{3}^{\tau 4} C}^{\prime}= & \frac{-1}{p^{b} k^{y}} \sum_{\omega_{m}} \frac{1}{\beta} \int \frac{d^{5} p_{3}}{(2 \pi)^{5}}\left(p_{3}^{b}\left(p_{3}\right)^{\tau 4} k^{y}\right) \\
& \times\left(\frac{1}{2\left(i \omega^{2}-E_{-p_{3}}^{2}\right)\left(i \omega^{2}-E_{p+p_{3}}^{2}\right)\left(i \omega^{2}-E_{k+p+p_{3}}^{2}\right)}\right) \\
& \left.+\frac{1}{2\left(i \omega^{2}-E_{k-p_{3}}^{2}\right)\left(i \omega^{2}-E_{-p_{3}}^{2}\right)\left(i \omega^{2}-E_{p+p_{3}}^{2}\right)}\right) \\
= & \int \frac{d^{5} p_{3}}{(2 \pi)^{5}} \frac{i p_{3}^{b}}{2 p^{b}}\left(\mathcal{M}_{1}+\mathcal{M}_{2}\right) \\
I_{p_{3}^{\tau 2} D}^{\prime}= & \int \frac{d^{5} p_{3}}{(2 \pi)^{5}} \frac{-i p_{3}^{y}\left(p_{3}^{\tau}\right)^{2}\left(p_{3}^{a 2}+p_{3}^{b^{2}}+p_{3}^{y 2}+p_{3}^{x 2}+p_{3}^{z 2}\right)}{2 k^{y}} \\
& \times\left(\frac{1}{2\left(i \omega^{2}-E_{-p_{3}}^{2}\right)\left(i \omega^{2}-E_{p+p_{3}}^{2}\right)\left(i \omega^{2}-E_{k+p+p_{3}}^{2}\right)}\right. \\
& \left.+\frac{1}{2\left(i \omega^{2}-E_{k-p_{3}}^{2}\right)\left(i \omega^{2}-E_{-p_{3}}^{2}\right)\left(i \omega^{2}-E_{p+p_{3}}^{2}\right)}\right) \\
= & \int \frac{d^{5} p_{3}}{(2 \pi)^{5}} \frac{i p_{3}^{y}\left(p_{3}^{a 2}+p_{3}^{b^{2}}+p_{3}^{y 2}+p_{3}^{x 2}+p_{3}^{z 2}\right)}{4 k^{y}}\left(\mathcal{M}_{3}+\mathcal{M}_{4}\right) .
\end{aligned}
$$

The Matsubara sums can be performed by the following formulae

$$
\begin{aligned}
\mathcal{M}_{1}= & \frac{1}{\beta} \sum_{m} \frac{(i \omega)^{4}}{\left(i \omega_{m}^{2}-E_{-p_{3}}^{2}\right)\left(i \omega_{m}^{2}-E_{p+p_{3}}^{2}\right)\left(i \omega_{m}^{2}-E_{k+p+p_{3}}^{2}\right)}, \\
= & \frac{-E_{p_{3}+p+k}^{3} b\left(E_{p_{3}+p+k}\right)}{\left(E_{p_{3}+p+k}^{2}-E_{p_{3}}^{2}\right)\left(E_{p_{3}+p+k}^{2}-E_{p_{3}+p}^{2}\right)}+\frac{-E_{p_{3}+p}^{3} b\left(E_{p_{3}+p}\right)}{\left(E_{p_{3}+p}^{2}-E_{p_{3}+p+k}^{2}\right)\left(E_{p_{3}+p}^{2}-E_{p_{3}}^{2}\right)} \\
& +\frac{-E_{p_{3}}^{3} b\left(E_{p_{3}}\right)}{\left(E_{p_{3}}^{2}-E_{p_{3}+p+k}^{2}\right)\left(E_{p_{3}}^{2}-E_{p_{3}+p}^{2}\right)} . \\
\mathcal{M}_{2}= & \frac{1}{\beta} \sum_{m} \frac{(i \omega)^{4}}{\left(i \omega_{m}^{2}-E_{k-p_{3}}^{2}\right)\left(i \omega_{m}^{2}-E_{p_{3}}^{2}\right)\left(i \omega_{m}^{2}-E_{p+p_{3}}^{2}\right)}, \\
= & \frac{-E_{p_{3}}^{3} b\left(E_{p 3}\right)}{\left(E_{p_{3}}^{2}-E_{p_{3}-k}^{2}\right)\left(E_{p_{3}}^{2}-E_{p_{3}+p}^{2}\right)}+\frac{-E_{p_{3}+p}^{3} b\left(E_{p_{3}+p}\right)}{\left(E_{p_{3}+p}^{2}-E_{p_{3}-k}^{2}\right)\left(E_{p_{3}+p}^{2}-E_{p_{3}}^{2}\right)} \\
& +\frac{-E_{p_{3}-k}^{3} b\left(E_{p_{3}-k}\right)}{\left(E_{p_{3}-k}^{2}-E_{p_{3}+p}^{2}\right)\left(E_{p_{3}-k}^{2}-E_{p_{3}}^{2}\right)} .
\end{aligned}
$$




$$
\begin{aligned}
\mathcal{M}_{3}= & \frac{1}{\beta} \sum_{m} \frac{(i \omega)^{2}}{\left(i \omega_{m}^{2}-E_{-p_{3}}^{2}\right)\left(i \omega_{m}^{2}-E_{p+p_{3}}^{2}\right)\left(i \omega_{m}^{2}-E_{k+p+p_{3}}^{2}\right)}, \\
= & \frac{-E_{p 3+p+k} b\left(E_{p_{3}+p+k}\right)}{\left(E_{p_{3}+p+k}^{2}-E_{p_{3}}^{2}\right)\left(E_{p_{3}+p+k}^{2}-E_{p_{3}+p}^{2}\right)}+\frac{-E_{p_{3}+p} b\left(E_{p_{3}+p}\right)}{\left(E_{p_{3}+p}^{2}-E_{p_{3}+p+k}^{2}\right)\left(E_{p_{3}+p}^{2}-E_{p_{3}}^{2}\right)} \\
& +\frac{-E_{p_{3}} b\left(E_{p_{3}}\right)}{\left(E_{p_{3}}^{2}-E_{p_{3}+p+k}^{2}\right)\left(E_{p_{3}}^{2}-E_{p_{3}+p}^{2}\right)} . \\
\mathcal{M}_{4}= & \frac{1}{\beta} \sum_{m} \frac{(i \omega)^{2}}{\left(i \omega_{m}^{2}-E_{k-p_{3}}^{2}\right)\left(i \omega_{m}^{2}-E_{p_{3}}^{2}\right)\left(i \omega_{m}^{2}-E_{p+p_{3}}^{2}\right)}, \\
= & \frac{-E_{p 3} b\left(E_{p 3}\right)}{\left(E_{p_{3}}^{2}-E_{p_{3}-k}^{2}\right)\left(E_{p_{3}}^{2}-E_{p_{3}+p}^{2}\right)}+\frac{-E_{p_{3}+p} b\left(E_{p_{3}+p}\right)}{\left(E_{p_{3}+p}^{2}-E_{p_{3}-k}^{2}\right)\left(E_{p_{3}+p}^{2}-E_{p_{3}}^{2}\right)} \\
& +\frac{-E_{p_{3}-k} b\left(E_{p_{3}-k}\right)}{\left(E_{p_{3}-k}^{2}-E_{p_{3}+p}^{2}\right)\left(E_{p_{3}-k}^{2}-E_{p_{3}}^{2}\right)} .
\end{aligned}
$$

Performing the usual manipulations we obtain

$$
\begin{aligned}
\lim _{p^{b}, k^{y} \rightarrow 0}\left(I_{p_{3}^{\tau 4} C}^{\prime}+I_{p_{3}^{\tau 2} D}^{\prime}\right) & =\int \frac{d^{5} p_{3}}{(2 \pi)^{5}} \frac{-i}{2} \frac{E_{p_{3}}^{3} b\left(E_{p_{3}}\right)}{8 E_{p_{3}}^{4} \sin ^{2} \phi_{1} \cos ^{2} \phi_{2} \sin ^{2} \phi_{1}}, \\
& =\frac{i \pi T^{4}}{2 \times 32 \times 15} .
\end{aligned}
$$

Similarly we have,

$$
\begin{aligned}
& \hat{I}_{p_{3}^{a 4}}=\hat{I}_{p_{3}^{y 4}}=\hat{I}_{p_{3}^{z^{4}}}=\frac{i \pi T^{4}}{2 \times 32 \times 15} . \\
& \hat{I}_{p_{3}^{b 4}}=\frac{5 i \pi T^{4}}{2 \times 32 \times 15} .
\end{aligned}
$$

Summing up all contributions of $M_{10}$ and $M_{11}$ ) we obtain

$$
\lim _{p^{b}, k^{y} \rightarrow 0}\left(I_{M_{10}}+I_{M_{11}}\right)=\frac{10 i \pi T^{4}}{15 \times 32} .
$$

Finally let put all the finite contributions together, from (B.58) and (B.80),

$$
\lim _{p^{b}, k^{y} \rightarrow 0}\left(I_{M_{10}}+I_{M_{11}}+I_{M_{8 A}}+I_{M_{8 B}}\right)=\frac{-16 i \pi T^{4}}{15 \times 32} .
$$

From (5.4) and (5.6) we have

$$
\lambda_{3}^{(6)}=\frac{3}{64} \frac{16 \pi T^{4}}{15} \text {. }
$$

Vanishing of contact terms. We show that the contribution of contact terms in the (5.9) to the transport coefficient vanish. We have 4 sets of contact terms

$$
\begin{array}{ll}
C_{1}=\left\langle T^{\tau a}(p+k) \frac{\delta T^{\tau x}(-k)}{\sqrt{g} \delta h_{\tau z}(p)}\right\rangle_{E}, & C_{2}=\left\langle\frac{\delta T^{\tau a}(p+k)}{\sqrt{g} \delta h_{\tau z}(p)} T^{\tau x}(-k)\right\rangle_{E} \\
C_{3}=\left\langle\frac{\delta T^{\tau a}(p+k)}{\sqrt{g} \delta h_{\tau x}(k)} T^{\tau z}(-p)\right\rangle_{E}, & C_{4}=\left\langle\frac{\delta^{2} T^{\tau a}(p+k)}{\sqrt{g} \delta h_{\tau z}(p) \sqrt{g} \delta h_{\tau x}(p)}\right\rangle_{E} .
\end{array}
$$


We show that the contact terms do not contribute to the three point function. We begin our analysis with the term $C_{1}$. It is sufficient to impose the self dual condition on just one of the vertices in the diagram. To obtain the relevant contact terms we expand the stress tensors to first order in metric perturbations. We evaluate contact term $C_{1}$ first. We impose the self dual condition in the stress tensor $T^{\tau a}$ and expand $T^{\tau x}$ to first order in metric perturbations.

$$
\begin{aligned}
T^{\tau a}(p+k)= & \sum_{\omega_{m}} \frac{-1}{\beta} \int \frac{d^{5} p_{1}}{(2 \pi)^{5}} \frac{1}{2}\left(F^{\tau b x}+i F^{a y z}\right)\left(-p_{1}+p+k\right)\left(F^{a b x}-i F^{\tau y z}\right)\left(p_{1}\right) \\
& +\frac{1}{2}\left(F^{\tau b y}-i F^{a x z}\right)\left(-p_{1}+p+k\right)\left(F^{a b y}+i F^{\tau x z}\right)\left(p_{1}\right) \\
& +\frac{1}{2}\left(F^{\tau b z}-i F^{a y x}\right)\left(-p_{1}+p+k\right)\left(F^{a b z}+i F^{\tau y x}\right)\left(p_{1}\right) .
\end{aligned}
$$

To extract the derivative of $T^{\tau x}$ with respect to the metric perturbation, let us write it as

$$
\begin{aligned}
T^{\tau x} & =-\frac{1}{2} F^{\tau a b} F_{a b}^{x}, \\
& =\frac{-1}{2} g^{\tau \alpha_{1}} g^{a \alpha_{2}} g^{b \alpha_{3}} g^{x \alpha_{4}} F_{\alpha_{1} \alpha_{2} \alpha_{3}} F_{\alpha_{4} a b} .
\end{aligned}
$$

where we expand $g^{\mu \nu}$ upto 2nd order in metric perturbations using the expansion

$$
g_{\mu \nu}=-\delta_{\mu \nu}+h_{\mu \nu}
$$

The metric perturbations we turn on are $h_{\tau x}, h_{\tau z}, h_{\tau a}$ only. Fourier transforming the resulting expression and taking the derivative with the metric perturbations yields

$$
\begin{aligned}
\frac{\delta T^{\tau x}(-k)}{\delta h_{\tau z}(p)}= & -\int \frac{d^{5} p_{3}}{(2 \pi)^{5}}\left[F^{z a b}\left(-p_{3}-p-k\right) F^{x a b}\left(p_{3}\right)+F^{z a y}\left(-p_{3}-p-k\right) F^{x a y}\left(p_{3}\right)\right. \\
& \left.+F^{z b y}\left(-p_{3}-p-k\right) F^{x b y}\left(p_{3}\right)\right] .
\end{aligned}
$$

After performing the Wick contractions from (5.12) and (B.87) we get,

$$
\begin{aligned}
C_{1}= & {\left[i p_{3}^{b} k^{y}\left(\left(p_{3}^{a}\right)^{2}+\left(p_{3}^{b}\right)^{2}-\left(p_{3}^{\tau}\right)^{2}+\left(p_{3}^{x}\right)^{2}+\left(p_{3}^{y}\right)^{2}+\left(p_{3}^{z}\right)^{2}\right)+i p_{3}^{b}\left(k^{y}\right)^{2} p_{3}^{y}\right] } \\
& +\left[-i\left(p_{3}^{a}\right)^{2} p_{3}^{y}-i\left(p_{3}^{b}\right)^{2} p_{3}^{y}+i\left(p_{3}^{\tau}\right)^{2} p_{3}^{y}-i\left(p_{3}^{x}\right)^{2} p_{3}^{y}-i p_{3}^{y}\left(p_{3}^{z}\right)^{2}\right. \\
& \left.-i\left(p_{3}^{y}\right)^{3}+k^{y}\left(i\left(p_{3}^{b}\right)^{2}-i\left(p_{3}^{y}\right)^{2}\right)\right] p^{b}-i\left(p^{b}\right)^{2} p_{3}^{b} p_{3}^{y} \\
& \times \frac{-1}{\left(i \omega^{2}-E_{p_{3}}^{2}\right)\left(i \omega^{2}-E_{k+p+p_{3}}^{2}\right)} .
\end{aligned}
$$

Let us first analyse the terms which are linear in $p^{b} k^{y}$.

$$
C_{1,14}+C_{1,15}=\frac{-i}{2 \beta} \sum_{m} \int \frac{d^{5} p_{3}}{(2 \pi)^{5}} \frac{\left(p_{3}^{b}\right)^{2}-\left(p_{3}^{y}\right)^{2}}{\left(i \omega^{2}-E_{p_{3}}^{2}\right)\left(i \omega^{2}-E_{k+p+p_{3}}^{2}\right)} .
$$


Here we have labelled the terms using the order they occur in (B.88) and also divided by $p^{b} k^{y}$. The sum over Matsubara frequencies is done by the following formula

$$
\frac{1}{\beta} \sum_{m} \frac{1}{\left(i \omega^{2}-E_{p_{3}}^{2}\right)\left(i \omega^{2}-E_{k+p+p_{3}}^{2}\right)}=-\left(\frac{b\left(p_{3}\right)}{E_{p_{3}}\left(E_{p_{3}}^{2}-E_{p_{3}+p+k}^{2}\right)}+\frac{b\left(p_{3}+p+k\right)}{E_{p_{3}+p+k}\left(E_{p_{3}+p+k}^{2}-E_{p_{3}}^{2}\right)}\right) .
$$

Therefore we have

$$
\begin{aligned}
C_{1,14}+C_{1,15}= & \frac{-i}{2} \int \frac{d^{5} p_{3}}{(2 \pi)^{5}}\left(\left(p_{3}^{b}\right)^{2}-\left(p_{3}^{y}\right)^{2}\right)\left(-\frac{b\left(p_{3}\right)}{E_{p_{3}}\left(E_{p_{3}}^{2}-E_{p_{3}+p+k}^{2}\right)}\right. \\
& \left.-\frac{b\left(p_{3}+p+k\right)}{E_{p_{3}+p+k}\left(E_{p_{3}+p+k}^{2}-E_{p_{3}}^{2}\right)}\right) .
\end{aligned}
$$

We proceed to shift the variables so that both the numerators in the Matsubara sum are functions of the internal momentum $p_{3}$. This implies the shift of variables $p_{3} \rightarrow-p_{3}-p-k$ in the second term of the matsubara sum.

$$
\begin{aligned}
C_{1,14}+C_{1,15}= & \frac{-i}{2} \int \frac{d^{5} p_{3}}{(2 \pi)^{5}}\left(\frac{\left(-2\left(p_{3}^{b}\right)^{2}-2 p_{3}^{b} p^{b}-\left(p^{b}\right)^{2}\right) b\left(p_{3}\right)}{E_{p_{3}}\left(E_{p_{3}}^{2}-E_{p_{3}+p+k}^{2}\right)}\right. \\
& \left.+\frac{\left(2\left(p_{3}^{y}\right)^{2}+2 p_{3}^{y} k^{y}+\left(k^{y}\right)^{2}\right) b\left(p_{3}\right)}{E_{p_{3}}\left(E_{p_{3}}^{2}-E_{p_{3}+p+k}^{2}\right)}\right) .
\end{aligned}
$$

We proceed to take the limit $p^{b}, k^{y} \rightarrow 0$. The result is independent of the order in which the limits are taken,

$$
\lim _{p^{b}, k^{y} \rightarrow 0}\left(I_{1,14}+I_{1,15}\right)=\frac{i}{2} \int \frac{d^{5} p_{3}}{(2 \pi)^{5}} \frac{b\left(p_{3}\right)}{2 p_{3}}\left(-1-\frac{\sin ^{2} \phi_{1} \cos ^{2} \phi_{2}}{\cos ^{2} \phi_{1}}\right) .
$$

The angular integrals are performed using the $i \epsilon$ prescription, which yields

$$
\int_{0}^{\pi} \frac{\sin ^{5} \phi}{\cos ^{2} \phi}=-\frac{16}{3}
$$

Therefore we obtain

$$
C_{1,14}+C_{1,15}=0 .
$$

By an analysis similar to the cancellation in B.91, we can show that, in the limit $p^{b}, k^{y} \rightarrow 0$

$$
\begin{aligned}
C_{1,7}+C_{1,16} & =\frac{-1}{p^{b} k^{y}} \frac{i}{2 \beta} \sum_{m} \int \frac{d^{5} p_{3}}{(2 \pi)^{5}} \frac{p_{3}^{b}\left(k^{y}\right)^{2} p_{3}^{y}-p_{3}^{b}\left(p^{b}\right)^{2} p_{3}^{y}}{\left(i \omega^{2}-E_{p_{3}}^{2}\right)\left(i \omega^{2}-E_{k+p+p_{3}}^{2}\right)}, \\
\lim _{p^{b}, k^{y} \rightarrow 0}\left(C_{1,7}+C_{1,16}\right) & =0 .
\end{aligned}
$$

Let us now proceed to analyse the rest of the terms in $C_{1}$. Consider the terms

$$
C_{1,1}+C_{1,8}=\frac{-1}{p^{b} k^{y}} \frac{i}{2 \beta} \sum_{m} \int \frac{d^{5} p_{3}}{2 \pi^{5}} \frac{\left(k^{y} p_{3}^{b}\left(p_{3}^{a}\right)^{2}-p^{b} p_{3}^{y}\left(p_{3}^{a}\right)^{2}\right)}{\left(i \omega^{2}-E_{p_{3}}^{2}\right)\left(i \omega^{2}-E_{k+p+p_{3}}^{2}\right)} .
$$


Performing the Matsubara sum we have,

$$
\begin{aligned}
C_{1,1}+C_{1,8}= & \frac{-1}{p^{b} k^{y}} \frac{i}{2} \int \frac{d^{5} p_{3}}{2 \pi^{5}}\left(k^{y} p_{3}^{b}\left(p_{3}^{a}\right)^{2}-p^{b} p_{3}^{y}\left(p_{3}^{a}\right)^{2}\right)\left(-\frac{b\left(p_{3}\right)}{E_{p_{3}}\left(E_{p_{3}}^{2}-E_{p_{3}+p+k}^{2}\right)}\right. \\
& \left.-\frac{b\left(p_{3}+p+k\right)}{E_{p_{3}+p+k}\left(E_{p_{3}+p+k}^{2}-E_{p_{3}}^{2}\right)}\right)
\end{aligned}
$$

Shifting the internal momentum results in

$$
\begin{aligned}
C_{1,1}+C_{1,8} & =\frac{-i}{2} \int \frac{d^{5} p_{3}}{(2 \pi)^{5}}\left(\frac{\left(p_{3}^{a}\right)^{2}\left(-p^{b}\right)}{-p^{b}}-\frac{\left(p_{3}^{a}\right)^{2}\left(-k^{y}\right)}{-k^{y}}\right) \frac{b\left(p_{3}\right)}{\left(E_{p_{3}}^{2}-E_{p_{3}+p+k}^{2}\right)}, \\
& =0 .
\end{aligned}
$$

This pattern of cancellation occurs for the following terms in $C_{1}$

$$
C_{1,3}+C_{1,10}=C_{1,4}+C_{1,11}=C_{1,6}+C_{1,12}=0 .
$$

Similar analysis show that,

$$
\begin{aligned}
C_{1,2}+C_{1,5}+C_{1,9}+C_{1,13} & =\frac{-1}{p^{b} k^{y}} \frac{i}{2 \beta} \sum_{m} \int \frac{d^{5} p_{3}}{(2 \pi)^{5}} \frac{k^{y}\left(p_{3}^{b}\right)^{3}+k^{y}\left(p_{3}^{b}\right)^{2} p_{3}^{y}-p^{b}\left(p_{3}^{y}\right)^{3}-p^{b}\left(p_{3}^{y}\right)^{2} p_{3}^{b}}{\left(i \omega^{2}-E_{p_{3}}^{2}\right)\left(i \omega^{2}-E_{k+p+p_{3}}^{2}\right)} \\
\lim _{p^{b}, k^{y} \rightarrow 0} C_{1,2}+C_{1,5}+C_{1,9}+C_{1,13} & =0 .
\end{aligned}
$$

Therefore from all of the above results we conclude that the contact term $C_{1}$ vanishes.

$$
C_{1}=0 \text {. }
$$

We examine the term $C_{2}$. We impose the self dual condition in $T^{\tau x}$ and expand $T^{\tau a}$ to first order in metric perturbations.

$$
\begin{aligned}
T^{\tau x}(-k)= & \frac{-1}{2} \sum_{m} \frac{1}{\beta} \int \frac{d^{5} p_{3}}{(2 \pi)^{5}}\left(F^{\tau b a}-i F^{x y z}\right)\left(-p_{3}-k\right)\left(F^{x b a}+i F^{\tau y z}\right) \\
& +\left(F^{\tau b y}+i F^{x a z}\right)\left(-p_{3}-k\right)\left(F^{x b y}-i F^{\tau a z}\right) \\
& +\left(F^{\tau b z}+i F^{x y a}\right)\left(-p_{3}-k\right)\left(F^{x b z}-i F^{\tau y a}\right) . \\
\frac{\delta T^{\tau a}(p+k)}{\delta h_{\tau z}(p)}= & -\frac{\sum_{m}}{\beta} \int \frac{d^{5} p_{3}}{(2 \pi)^{5}}\left(F^{z b x}\left(-p_{1}+k\right) F^{a b x}\left(p_{1}\right)+F^{z b y}\left(-p_{1}+k\right) F^{a b y}\left(p_{1}\right)\right. \\
& \left.F^{z x y}\left(-p_{1}+k\right) F^{a x y}\left(p_{1}\right)\right) .
\end{aligned}
$$

From (B.83), we have

$$
C_{2}=\frac{-1}{p k} \frac{-i}{\beta} \sum_{m} \frac{d^{5} p_{3}}{(2 \pi)^{5}} \frac{\left(\left(p_{3}^{a}\right)^{2} p_{3}^{b}-\left(p_{3}^{z}\right)^{2} p_{3}^{b}\right) k}{\left(i \omega^{2}-E_{p_{3}}^{2}\right)\left(i \omega^{2}-E_{k+p_{3}}^{2}\right)} .
$$


Performing the same analysis as done for $C_{1}$ we find that,

$$
C_{2}=0 \text {. }
$$

Similarly we get

$$
C_{3}=0 .
$$

The cancellation of the $C_{4}$ term is more easy to see

$$
C_{4}=\left\langle\frac{\delta^{2} T^{\tau a}(p+k)}{\sqrt{g} \delta h_{\tau z}(p) \sqrt{g} \delta h_{\tau x}(p)}\right\rangle_{E} .
$$

We have to expand the stress tensor to second order in metric perturbations and extract out the $h_{\tau x} h_{\tau z}$ coefficient. The component of stress tensor of interest is given by

$$
\begin{aligned}
T^{\tau a}= & \frac{-1}{\beta} \sum_{\omega_{n}} \int \frac{d^{5} p_{1}}{(2 \pi)^{5}}\left(\tilde{F}^{\tau b x}\left(-p_{1}+p+k\right) F_{b x}^{a}\left(p_{1}\right)+\tilde{F}^{\tau x y}\left(-p_{1}+p+k\right) F_{x y}^{a}\left(p_{1}\right)\right. \\
& \tilde{F}^{\tau y z}\left(-p_{1}+p+k\right) F_{y z}^{a}\left(p_{1}\right)+\tilde{F}^{\tau b z}\left(-p_{1}+p+k\right) F_{b z}^{a}\left(p_{1}\right)+\tilde{F}^{\tau x z}\left(-p_{1}+p+k\right) F_{x z}^{a}\left(p_{1}\right) \\
& \left.+\tilde{F}^{\tau b y}\left(-p_{1}+p+k\right) F_{b y}^{a}\left(p_{1}\right)\right) .
\end{aligned}
$$

Here we have written down the stress tensor with the self dual projection on one of the fields strengths. Since the contractions involved are all self contractions with the stress tensor, this is sufficient. ${ }^{13}$ Only the terms which are quadratic in $h_{\mu \nu}$, more specifically $h_{\tau x} h_{\tau z}$ contribute to the correlator.

$$
\begin{aligned}
\tilde{F}^{\tau b x} & =\frac{1}{2}\left(g^{\tau \alpha_{1}} g^{b \alpha_{2}} g^{x \alpha_{3}} F_{\alpha_{1} \alpha_{2} \alpha_{3}}-i F_{y z a}\right), \\
& =\frac{1}{2}\left(-F_{\tau b x}-i F_{y z a}-h_{\tau z} F_{z b x}\right)+O\left(h^{3}\right) \\
F_{b x}^{a} & =-F_{a b x}+\quad O\left(h^{3}\right) .
\end{aligned}
$$

Therefore we see that there are no terms of the kind $h_{\tau x} h_{\tau z}$ in the metric expansion of $T^{\tau a}$. Thus we obtain

$$
C_{4}=0 .
$$

This concludes our analysis which shows that the contribution to the transport coefficient from the contact terms vanish.

\section{Summary of $\eta$ invariants in various dimensions}

We have seen that the $\eta$ invariant corresponding to the $T^{2}$ transformation determines the contribution of the chiral matter to the parity odd transport coefficient. Since evaluation of the $\eta$ invariant is an involved exercise it is convenient to turn the problem around and evaluate the $\eta$ invariant using the data provided by the transport coefficient. In this

\footnotetext{
${ }^{13}$ We have also carried out the analysis with the self dual condition imposed on both the field strength that occurs in the stress tensor. The final result is $C_{4}=0$
} 


\begin{tabular}{|c|c|c|}
\hline Dimension & Species & $\eta$ invariant (upto mod 2) \\
\hline$d=2$ & Fermions & $\frac{1}{6}$ \\
\hline & Gravitinos & $\frac{1}{6}$ \\
\hline & Chiral Bosons & $\frac{1}{6}$ \\
\hline \multirow{2}{*}{$d=6$} & Fermions & $-\frac{7}{60} n m$ \\
\hline & Gravitinos & $-\frac{35}{60} n m$ \\
\hline \multirow{2}{*}{$d=10$} & Self Dual Tensors & $-\frac{16}{60} n m$ \\
\hline & Fermions & $\frac{31}{126} m n o p$ \\
\hline & Gravitinos & $\frac{279}{126} m n o p$ \\
\hline & Self Dual Tensors & $\frac{256}{126} m n o p$ \\
\hline
\end{tabular}

Table 1. $\eta$ invariants in various dimensions.

appendix we first summarise the $\eta$ invariants of fermions, gravitinos and self dual tensors in $d=2,6$ dimensions. We then use the knowledge of anomalous transport given in [22] to obtain the $\eta$ invariants for various species in $d=10$. Our starting point is the metric $g^{2 d}$ of the torus $\hat{T}^{2 d}$.

$$
d s_{2 d}^{2}=\left(d t+a_{1}\left(x_{1}\right) d x^{1}+a_{2}\left(x_{3}\right) d x^{2} \cdots a_{2 d-2}\left(x_{2 d-1}\right) d x^{2 d-2}\right)^{2}+d x_{1}^{2}+d x_{2}^{2} \cdots d x_{2 d-1}^{2}
$$

where the coordinates are periodic with period $2 \pi$. Fermionic matter along the torus directions have anti-periodic boundary conditions. This results in a non trivial field configuration for the metric components $a_{i}$ for $i=2,4, \cdots(2 d-2)$

$$
\begin{aligned}
x^{j} & \sim x^{j}+2 \pi, & j & =3,5, \cdots 2 d-1, \\
a_{i}\left(x^{j}\right) & =2 n \frac{x^{j}}{2 \pi}, & n & \in \mathbb{Z}
\end{aligned}
$$

Such a winding configuration ensures that under a $T^{2}$ transformation in the $\left(x^{i}, x^{j}\right)$ plane, as $x^{j} \rightarrow x^{j}+2 \pi, a_{i} \rightarrow a_{i}+2 n$. Thus the boundary conditions $(A, A)$ remain invariant along these directions. We wish to evaluate the $\eta$ invariant corresponding to the $T^{2}$ transformation under which $a_{1} \rightarrow a_{1}+2$. The effective action that one gets from computing the $\eta$ invariant in such a set up must reproduce the correct value for the parity odd transport coefficients in the de-compactification limit. Using the values of anomalous transport coefficients determined by the pure gravitational anomaly in arbitrary dimensions given in [22] we have calculated the $\eta$ invariants of various species of matter in various dimensions. They are summarized in table 1 , where $m, n, o, p \in \mathbb{Z}$, denote the non-trivial winding number of the metric components. Note that all these $\eta$ invariants are determined to mod 2.

Open Access. This article is distributed under the terms of the Creative Commons Attribution License (CC-BY 4.0), which permits any use, distribution and reproduction in any medium, provided the original author(s) and source are credited. 


\section{References}

[1] S. Bhattacharyya, S. Lahiri, R. Loganayagam and S. Minwalla, Large rotating AdS black holes from fluid mechanics, JHEP 09 (2008) 054 [arXiv:0708.1770] [INSPIRE].

[2] J. Erdmenger, M. Haack, M. Kaminski and A. Yarom, Fluid dynamics of R-charged black holes, JHEP 01 (2009) 055 [arXiv:0809.2488] [INSPIRE].

[3] N. Banerjee, J. Bhattacharya, S. Bhattacharyya, S. Dutta, R. Loganayagam and P. Surowka, Hydrodynamics from charged black branes, JHEP 01 (2011) 094 [arXiv:0809.2596] [INSPIRE].

[4] D.T. Son and P. Surowka, Hydrodynamics with Triangle Anomalies, Phys. Rev. Lett. 103 (2009) 191601 [arXiv:0906.5044] [INSPIRE].

[5] Y. Neiman and Y. Oz, Relativistic Hydrodynamics with General Anomalous Charges, JHEP 03 (2011) 023 [arXiv: 1011.5107] [INSPIRE].

[6] N. Banerjee, J. Bhattacharya, S. Bhattacharyya, S. Jain, S. Minwalla and T. Sharma, Constraints on Fluid Dynamics from Equilibrium Partition Functions, JHEP 09 (2012) 046 [arXiv: 1203.3544] [INSPIRE].

[7] N. Banerjee, S. Dutta, S. Jain, R. Loganayagam and T. Sharma, Constraints on Anomalous Fluid in Arbitrary Dimensions, JHEP 03 (2013) 048 [arXiv: 1206.6499] [INSPIRE].

[8] K. Jensen, Triangle Anomalies, Thermodynamics and Hydrodynamics, Phys. Rev. D 85 (2012) 125017 [arXiv:1203.3599] [INSPIRE].

[9] K. Jensen, M. Kaminski, P. Kovtun, R. Meyer, A. Ritz and A. Yarom, Towards hydrodynamics without an entropy current, Phys. Rev. Lett. 109 (2012) 101601 [arXiv:1203.3556] [INSPIRE].

[10] S. Jain and T. Sharma, Anomalous charged fluids in $1+1 d$ from equilibrium partition function, JHEP 01 (2013) 039 [arXiv: 1203.5308] [INSPIRE].

[11] M. Valle, Hydrodynamics in 1+1 dimensions with gravitational anomalies, JHEP 08 (2012) 113 [arXiv: 1206.1538] [INSPIRE].

[12] K. Landsteiner, E. Megias and F. Pena-Benitez, Gravitational Anomaly and Transport, Phys. Rev. Lett. 107 (2011) 021601 [arXiv:1103.5006] [INSPIRE].

[13] K. Landsteiner, E. Megias, L. Melgar and F. Pena-Benitez, Holographic Gravitational Anomaly and Chiral Vortical Effect, JHEP 09 (2011) 121 [arXiv:1107.0368] [INSPIRE].

[14] K. Landsteiner, E. Megias and F. Pena-Benitez, Anomalous Transport from Kubo Formulae, Lect. Notes Phys. 871 (2013) 433 [arXiv:1207.5808] [INSPIRE].

[15] S. Golkar and D.T. Son, (Non)-renormalization of the chiral vortical effect coefficient, JHEP 02 (2015) 169 [arXiv: 1207.5806] [INSPIRE].

[16] S.D. Chowdhury and J.R. David, Anomalous transport at weak coupling, JHEP 11 (2015) 048 [arXiv : 1508.01608] [INSPIRE].

[17] K. Jensen, R. Loganayagam and A. Yarom, Thermodynamics, gravitational anomalies and cones, JHEP 02 (2013) 088 [arXiv: 1207.5824] [INSPIRE].

[18] K. Jensen, R. Loganayagam and A. Yarom, Chern-Simons terms from thermal circles and anomalies, JHEP 05 (2014) 110 [arXiv:1311.2935] [INSPIRE].

[19] L. Di Pietro and Z. Komargodski, Cardy formulae for SUSY theories in $d=4$ and $d=6$, JHEP 12 (2014) 031 [arXiv:1407.6061] [INSPIRE]. 
[20] S. Golkar and S. Sethi, Global Anomalies and Effective Field Theory, arXiv:1512.0260.

[21] L. Álvarez-Gaumé and E. Witten, Gravitational Anomalies, Nucl. Phys. B 234 (1984) 269 [INSPIRE].

[22] R. Loganayagam, Anomaly Induced Transport in Arbitrary Dimensions, arXiv:1106. 0277 [INSPIRE].

[23] R. Loganayagam and P. Surowka, Anomaly/Transport in an Ideal Weyl gas, JHEP 04 (2012) 097 [arXiv:1201.2812] [INSPIRE].

[24] R. Loganayagam, Anomalies and the Helicity of the Thermal State, JHEP 11 (2013) 205 [arXiv:1211.3850] [INSPIRE].

[25] E. Witten, Global gravitational anomalies, Commun. Math. Phys. 100 (1985) 197 [InSPIRE].

[26] T. Eguchi, P.B. Gilkey and A.J. Hanson, Gravitation, Gauge Theories and Differential Geometry, Phys. Rept. 66 (1980) 213 [INSPIRE].

[27] J.M. Maldacena and A. Strominger, $A d S_{3}$ black holes and a stringy exclusion principle, JHEP 12 (1998) 005 [hep-th/9804085] [INSPIRE].

[28] S. Monnier, The global gravitational anomaly of the self-dual field theory, Commun. Math. Phys. 325 (2014) 73 [arXiv:1110.4639] [INSPIRE].

[29] S. Monnier, The global anomaly of the self-dual field in general backgrounds, Annales Henri Poincaré 17 (2016) 1003 [arXiv: 1309.6642] [INSPIRE].

[30] L. Álvarez-Gaumé and P.H. Ginsparg, The Structure of Gauge and Gravitational Anomalies, Annals Phys. 161 (1985) 423 [Erratum ibid. 171 (1986) 233] [INSPIRE].

[31] T. Azeyanagi, R. Loganayagam, G.S. Ng and M.J. Rodriguez, Holographic Thermal Helicity, JHEP 08 (2014) 040 [arXiv: 1311.2940] [INSPIRE].

[32] S. Grozdanov and N. Poovuttikul, Universality of anomalous conductivities in theories with higher-derivative holographic duals, arXiv:1603.0877. 